\title{
A Survey on Coverage Enhancement in Cellular Networks: Challenges and Solutions for Future Deployments
}

\author{
Ruben Borralho*†, Abdelrahim Mohamed*, Atta Quddus*, Pedro Vieira ${ }^{\ddagger \S}$, Rahim Tafazolli* \\ ${ }^{*}$ Institute for Communication Systems ICS, Home of 5GIC, University of Surrey, Guildford, U.K. \\ E-mail: \{r.a.borralho, abdelrahim.mohamed, a.quddus, r.tafazolli\} @ surrey.ac.uk \\ ${ }^{\dagger}$ Celfinet - Outstanding Networks, Lisbon, Portugal \\ $¥$ Telecommunications Institute, Lisbon, Portugal

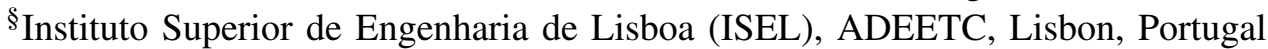 \\ E-mail: \{pedro.vieira\} @ isel.pt
}

\begin{abstract}
Seamless and ubiquitous coverage are key factors for future cellular networks. Despite capacity and data rates being the main topics under discussion when envisioning the Fifth Generation (5G) and beyond of mobile communications, network coverage remains one of the major issues since coverage quality highly impacts the system performance and end-user experience. The increasing number of base stations and user terminals is anticipated to negatively impact the network coverage due to increasing interference. Furthermore, the "ubiquitous coverage" use cases, including rural and isolated areas, present a significant challenge for mobile communication technologies. This survey presents an overview of the concept of coverage, highlighting the ways it is studied, measured, and how it impacts the network performance. Additionally, an overlook of the most important key performance indicators influenced by coverage, which may affect the envisioned use cases with respect to throughput, latency, and massive connectivity, are discussed. Moreover, the main existing developments and deployments which are expected to augment the network coverage, in order to meet the requirements of the emerging systems, are presented as well as implementation challenges.
\end{abstract}

Index Terms-Cellular networks, frequency bands, interference management, network coverage, network deployments, spectrum management.

\section{INTRODUCTION}

$\mathbf{N}$ OWADAYS, mobile networks represent one of the most technologically advanced areas. The exponential growth of subscribers together with the massive amount of consumed data and the user expectations and demand, take to the need to constantly improve the mobile network technologies. These improvements focus on reduced latency and network throughput and capacity maximization [1], [2]. These constant network enhancements are only possible through a growing community of engineers, academics, and stakeholders, who constantly work to accomplish the users needs. In this direction, new, optimized, and upgraded services are provided in short periods of time. The fifth-generation $(5 \mathrm{G})$ of mobile communications is not an exception to this trend. In fact, it elevates all these challenges into another level of complexity. New use cases and network architectures are being introduced, merging different technologies, providing powerful services, and promising significant improvements on data rates as well as minimal latency. All of this must be aligned with constrains regarding the specific environment characteristics, spectrum limitations, the required infrastructure investment, and energy consumption. Several new techniques and technologies are being suggested to meet these network performance requirements. Network access point (AP) densification, massive antennas with multiple-input-multiple-output (MIMO) solutions, or wider bandwidths by moving to millimeter wave (mm-Wave) spectrum can provide the desired augmented network capacity. In parallel, technologies such as softwaredefined networking or network functions virtualization can enable network flexibility, putting data centers closer to the end-user, and providing faster and more reliable solutions [3], [4].

Nevertheless, there are inherent implementation challenges regarding these technologies. Densification can significantly increase the network energy consumption [5], higher frequencies are susceptible to drastic attenuation due to increased path loss and channel intermittency, and centralization requires tremendous computational processing capacity [6]. In parallel, the network coverage challenges arise as one of the main concerns regarding future cellular networks. The notably expected benefits of future mobile telecommunication technologies for throughput and capacity cannot be fully achieved without providing high quality signals. Moreover, some of these technologies have coverage as the limiting factor. For example, in $\mathrm{mm}$-Waves based solutions, the coverage area is much smaller when compared to the legacy technologies. The deep indoor coverage also becomes a big challenge, which is essential for the massive machine-type communications (mMTC) use case. It is important to enable not only connectivity, but also to achieve enhanced network performance at those locations. Coverage in rural areas also becomes an important part with the requirements around the Universal Coverage concept [7]. The user density in some isolated areas does not justify the necessary operator investment in order to provide the desired quality of service (QoS). In addition to decreasing the network performance in terms of throughput, poor coverage planning 
also negatively impacts the so called low latency applications. Thus, a good mobile coverage is essential to achieve the initial $5 \mathrm{G}$ and beyond use cases. Additionally, there is the always present cell-edge problem, resulting from coverage holes or coverage overlap (which results in high interference) between cells of different sites or even within sectors of the same site.

\section{A. Related Works}

To the best knowledge of the authors, there is no indepth survey paper on the coverage of telecommunication networks. Nevertheless, some interesting works have been published targeting the challenges of future mobile technology requirements, including the network coverage as a sub-topic of the work. The analysis in [4] and [6] provides hints on the increasing demand for network coverage, suggesting solutions such as heterogeneous networks (HetNets), relaying, or cloudbased mechanisms for coverage enhancement in future mobile technologies. However, these are limited contributions in papers with the main focus on providing a broader view of the $5 \mathrm{G}$ enabling technologies. Some other works also investigate the trade-off between the network coverage and the network capacity and deployment costs [8], [9], [10]. In [11], the authors present a survey on coverage and connectivity of wireless sensor networks focusing on Internet-of-Things (IoT) paradigm.

The assessment of network coverage through mathematical (or numerical) analysis is also an area of intense research. In this regard, several studies such as [12] make use of stochastic geometry (SG) that provides a rich mathematical framework for performance analysis. In a more practical approach, there are some papers that provide coverage analysis based on field measurements [13], [14], [15], [16]. However, these models are not fully dedicated to the study of coverage alone since they also evaluate other metrics such as the network throughput, or focus on the coverage of specific techniques or technologies, such as mm-Waves or Coordinated Multi-Point (CoMP).

In a more general setting, some works provide valuable analysis (that also includes coverage discussion) of promising techniques to address the challenges of future cellular technology. These include surveys on densification [17], [18], [19], showing the increased connectivity capacity and potential of deploying different layers layers (overlay and underlay) of Base Stations (BSs) for improved network coverage in outdoor and indoor environments. Moreover, there are papers surveying Device-to-Device (D2D) communications [20], [21] and Unmanned Aerial Vehicles (UAVs) [22], [23], on their capabilities for relaying and network coverage extension. A similar analysis can also be found across the literature for mm-Waves [24], [25], [26], Distributed Antennas Systems (DASs) [27], beamforming [28], [29], [30], CoMP [31], [32], user equipment (UE) scheduling [33], and other interference mitigation solutions that positively impact the coverage [34], [35], [36]. Research work can also be found on solving particular issues of network coverage, such as the cell-edge and cell radius expansion [37]. Furthermore, studies exist in literature on mechanisms for coverage prediction, and exploring the different path loss models and the environments where they should be applied from a network planning perspective [38], [39]. These studies can also be complemented with reliable metrics for measuring the coverage [40].

As discussed in previous paragraphs, there are several works in literature that explore the impact of various techniques and methods on network coverage, however not in a holistic manner. This survey aims to fill this gap and provides an extensive and exhaustive discussion on this important topic in mobile networks. We aim to focus this survey entirely on network coverage, providing various definitions through which coverage can be measured, and identifying the main issues that limit coverage and potential solutions that can provide coverage enhancement, along with their benefits and drawbacks. This results in a holistic view and provides important insights into enhancing coverage for future mobile communication systems.

\section{B. Contributions}

This survey focuses on emerging mobile cellular network coverage advancements, showing the different perspectives discussed in the literature and focusing on real deployments targeting new mobile technologies. Several coverage definitions, coverage requirements expected from emerging cellular systems, key performance indicators (KPIs), and technologies for optimising coverage are discussed and analysed. It enables understanding of the existing trade-offs between coverage and other crucial parameters of mobile networks, such as capacity and latency. The contributions of this paper are summarised in the following:

- We provide a holistic view of network coverage starting with the definitions and culminating on the existing and promising mechanisms for coverage enhancement. Then we move towards the demanding coverage requirements of future cellular use cases.

- We explore the different approaches of studying and evaluating coverage in different sectors: the standardization bodies, the scientific literature, and industrial forums.

- We discuss the typical sources of coverage degradation and how these should be mitigated. In addition, this survey lists the main available planning tools in the market for proper management and planning of network coverage.

- We explore the main coverage related network requirements of the Third Generation Partnership Project (3GPP) Long Term Evolution (LTE) and 5G mobile technologies. We also discuss as to how several mechanisms of network coverage enhancement directly influence the achievement of these requirements.

- We define a new taxonomy for network coverage enhancement mechanisms divided into three main classes: network deployments, frequency bands, and interference management. Within each category, we survey techniques focusing on whether wide or short-range coverage is provided. We also explore the potential influence of these techniques on different use cases.

- When analysing a mechanism individually, we also comment on the possibility of integrating it with other potential mechanisms discussed in other sections of the paper. 
- Finally, we identify open issues and challenges together with potential future research directions.

\section{Paper Outline}

The remainder of this paper is organized as follows: Section II provides a holistic view on coverage, highlighting the available methods for studying and measuring it, the common network coverage issues as well as good practices regarding network planning to mitigate coverage problems. Section III focuses on mobile network requirements, and how coverage quality influences the accomplishment of these requirements. In Section IV, an overview of all principal network deployment mechanisms for coverage enhancement is provided, highlighting the benefits and implementation challenges. Section V addresses the influence of the different frequency bands on coverage, with special attention to the low frequency based solutions. In addition, it presents and discusses techniques to maximize coverage range in higher frequencies. In Section VI, the impact of interference management mechanisms on coverage is assessed, focusing on the benefits for cell-edge users. Section VIII concludes the paper and underlines future research directions. Table I provides a list for the acronyms used in the paper and Fig. 1 provides the organizational structure of the paper.

\section{Network Coverage: Definitions AND Challenges}

This section provides an overview of network coverage concept. It points out the main definitions used for network coverage and how it is evaluated in research studies, standards and forums. Additionally, some of the main coverage issues are explored and the main network KPIs related to coverage and targeting future mobile technologies are critically discussed.

\section{A. Coverage Definitions}

In general terms, coverage is provided through BS antennas spread across all worldwide territories, which enable the wireless telecommunications to end-users. Depending on the configuration, each BS covers a specific area in the surroundings of its localization. The size of this area is influenced by the BS transmit power, antenna tilt, carrier frequency, the deployment type, and by the surrounding environment. Elements such as mountains, hills, buildings and trees influence the range of the BS serving area. In this section, an overview of different coverage definitions is presented, focusing on three different telecommunications-related parties: standardization bodies, scientific literature and industrial forums. Rather than controversy, these different approaches allow assessing the coverage quality within the telecommunication networks in a very complete manner. This encompasses real network coverage dimensioning and evaluation, or analytical and simulation studies, through different network performance metrics.
1) Standardization Bodies: In the LTE, a BS is termed as enhanced NodeB (eNB). According to the $3 \mathrm{GPP}^{1}$ [41], the LTE eNB coverage area is defined by the Maximum Coupling Loss (MCL) parameter. It represents the limiting value of the coupling loss at which the service can be delivered, thus defining the coverage of the service. Considering this definition, the area around a BS that does not fulfill the MCL requirement, i.e., where the coupling loss is higher than the constraint value, is considered uncovered. For downlink (DL) and uplink (UL), the MCL is calculated as follows [41]:

$$
M C L_{D L}=T x_{D L}-S_{U E}
$$

and

$$
M C L_{U L}=T x_{U L}-S_{e N B}
$$

respectively, where $T x_{D L}$ and $T x_{U L}$ represents the maximum transmitted power in the DL and UL, respectively, and $S_{U E}$ and $S_{e N B}$ are the UE and eNB sensitivities, respectively.

The link budget is also considered a reasonable method for coverage projection, defined in the standards [42] and largely used in real network planning [13]. It quantifies the maximum allowable path loss (MAPL) between the transmitter and the receiver, taking into account all gain and loss factors occurring within the different interfaces in the system, from which the MCL can be calculated. The link budget provides the required signal-to-interference-plus-noise Ratio (SINR) operating point associated with a specific block error rate (BLER) target. The expected data rate at the cell-edge determines the critical coverage constraint, i.e., the cell-edge throughput specification determines the required SINR at which, the UE is either in or out of coverage. The system MAPL is defined by the minimum MAPL between the UL and DL connections. It is quantified as the difference between the transmitted effective isotropically radiated power (EIRP) and receiver radiated power. These radiated powers encompass both the transmitted and received power plus the respective transmit and receive antenna gains. This is the main difference when comparing MAPL with MCL, since the latter does not take into account the antenna gains [43]. Fig. 2 illustrates the main components behind the calculation of these metrics in the DL scenario. As one can see, the MCL is given by the difference between the transmitted power and the UE sensitivity, which is the sum of the required SINR, the receiver noise figure and the thermal noise power. In the MAPL metric, both transmitter and receiver antenna gains are also considered.

The MCL, MAPL and link budget are metrics used to assess the network coverage at the link-level. However, the 3GPP also defines some system-level KPIs for system performance evaluation, namely the $5^{t h}$-percentile of the throughput cumulative distribution function (CDF), $50^{t h}$-percentile of the throughput CDF, mean LTE UE throughput, and the tail perceived throughput [44]. Targeting network coverage, the $5^{t h}$-percentile throughput reflects the performance of the UE with the worst network conditions. Hence, it is tightly coupled with the cell coverage because it encompasses mainly the UE at the cell-edge, i.e., in the boundaries or limits of the cell area.

\footnotetext{
${ }^{1}$ Standards organization for mobile communications: $2 \mathrm{G}, 3 \mathrm{G}, \mathrm{LTE}$ and $5 \mathrm{G}$
} 


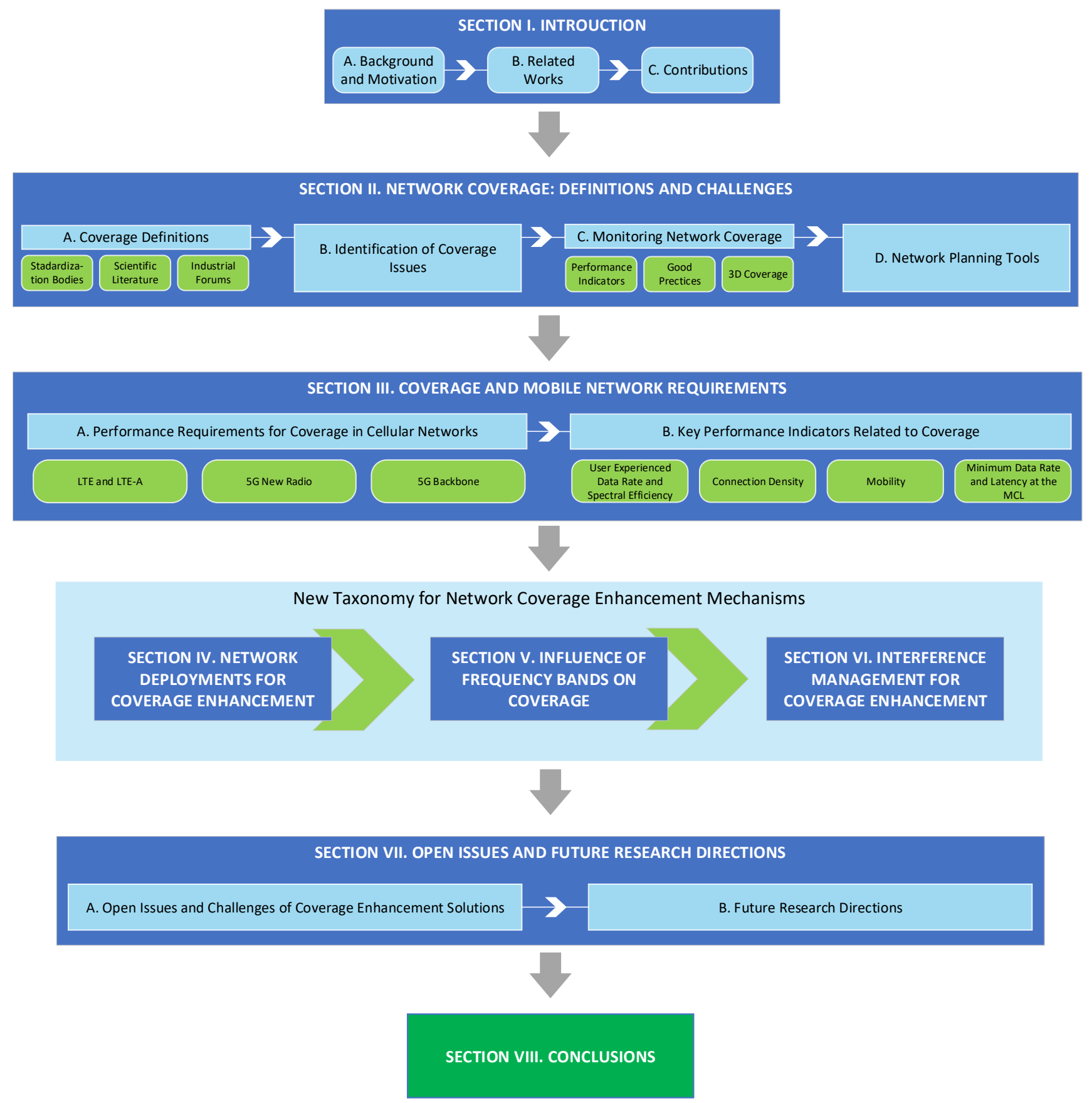

Figure 1. The organization and structure of the paper.

Additionally, the tail perceived throughput KPI measures the $5 \%$ worst perceived throughput among users, which is defined as the size of a burst divided by the time between the reception of the first and last packets from that burst [44]. In reality, coverage can be evaluated through the $5^{t h}$-percentile of SINR and spectral efficiency as well, since these KPIs also represent cell-edge users. In this direction, the KPIs measuring the worst user performance across the network are widely used to assess the network performance in terms of coverage enhancement deployments. This factor is observed in the explored studies in the following sections of this article.
The consortium for the 5G Infrastructure Public Private Partnership (5G PPP) runs several projects to deliver solutions, architectures and standards for the next generation of mobile communications. The 5G PPP defines the coverage area probability metric, which is defined as a percentage of the area under consideration where a certain service is provided with the necessary quality to the UE. This quality can be measured in terms of QoS or quality of experience (QoE) for any required parameter for the service to be delivered, whether it is data rate or latency. The coverage area probability is different for different service requirements of QoS/QoE [45]. 
Table I

LIST OF ACRONYMS.

\begin{tabular}{|c|c|c|c|}
\hline Acronym & Full form & Acronym & Full form \\
\hline $2 \mathrm{D}$ & Two-Dimensional & LTE-A & Long Term Eevolutio-Advanced \\
\hline $3 \mathrm{D}$ & Three-Dimensional & LTE-M & LTE-Machine Type Communication \\
\hline 3GPP & Third Generation Partnership Project & MAPL & Maximum Allowable Path Loss \\
\hline $5 \mathrm{G}$ & Fifth Generation & MCL & Maximum Coupling Loss \\
\hline AP & Access Point & MIMO & Multi Input Multi Output \\
\hline BER & Bit Error Rate & ML & Machine Learning \\
\hline BLER & Block Error Rate & mMIMO & massive MIMO \\
\hline $\mathrm{BS}$ & Base Station & mMTC & massive Machine-type Communication \\
\hline $\mathrm{CDF}$ & Cumulative Distribution Function & mm-Wave & millimeter wave \\
\hline $\mathrm{CF}$ & Cell-Free & MTC & Machine-type Communication \\
\hline CoMP & Coordinated Multi-Point & NB-IoT & Narrowband-IoT \\
\hline CQI & Channel Quality Indicator & NLoS & Non-Line-of-Sight \\
\hline CSI & Channel State Information & NOMA & Non-Orthogonal Multiple Access \\
\hline D2D & Device-to-Device & OFDMA & Orthogonal Frequency Division Multiple Access \\
\hline DAS & Distributed Antennas System & $\mathrm{OI}$ & Overload Indicator \\
\hline DL & Downlink & PM & Performance Management \\
\hline DT & Drive-test & QAM & Quadrature Amplitude Modulation \\
\hline E2E & End-to-End & QoE & Quality of Experience \\
\hline ELF & Extremely Low Frequency & QoS & Quality of Service \\
\hline eMBB & enhanced Mobile Broadband & QPSK & Quadrature Phase-shift Keying \\
\hline eNB & evolved NodeB & RAT & Radio Access Technology \\
\hline FDD & Frequency Division Duplex & $\mathrm{RB}$ & Resource Block \\
\hline FSK & Frequency Shift Keying & $\mathrm{RF}$ & Radio-frequency \\
\hline HAP & high-altitude platform & RIS & Reconfigurable Intelligent Surface \\
\hline HetNet & Heterogeneous Network & RS & Rate-Splitting \\
\hline $\mathrm{HF}$ & High Frequency & RSRP & Reference Signal Received Power \\
\hline HII & High Interference Indicator & RSS & Received Signal Strength \\
\hline HSDPA & High-Speed Downlink Packet Access & SG & Stochastic Geometry \\
\hline ICI & Inter-Cell Interference & SINR & Signal-to-Interference-plus-Noise Ratio \\
\hline ICIC & Inter-Cell Interference Coordination & SIR & Signal-to-Interference ratio \\
\hline IoT & Internet-of-Things & SNR & Signal-to-Noise Ratio \\
\hline KPI & Key Performance Indicator & SON & Self-Organizing Network \\
\hline LAP & Low-altitude platform & TDD & Time Division Duplex \\
\hline LEO & Low Earth orbit & UAV & Unmanned Aerial Vehicle \\
\hline $\mathrm{LiFi}$ & Light Fidelity & $\mathrm{UE}$ & User Equipment \\
\hline LoRa & Long Range & UHF & Ultra High Frequency \\
\hline LoS & Line-of-Sight & $\mathrm{UL}$ & Uplink \\
\hline LoRa & Long Range & URLLC & Ultra-Reliable and Low-Latency Communications \\
\hline LTE & Long Term Evolution & VLF & Very Low Frequency \\
\hline
\end{tabular}

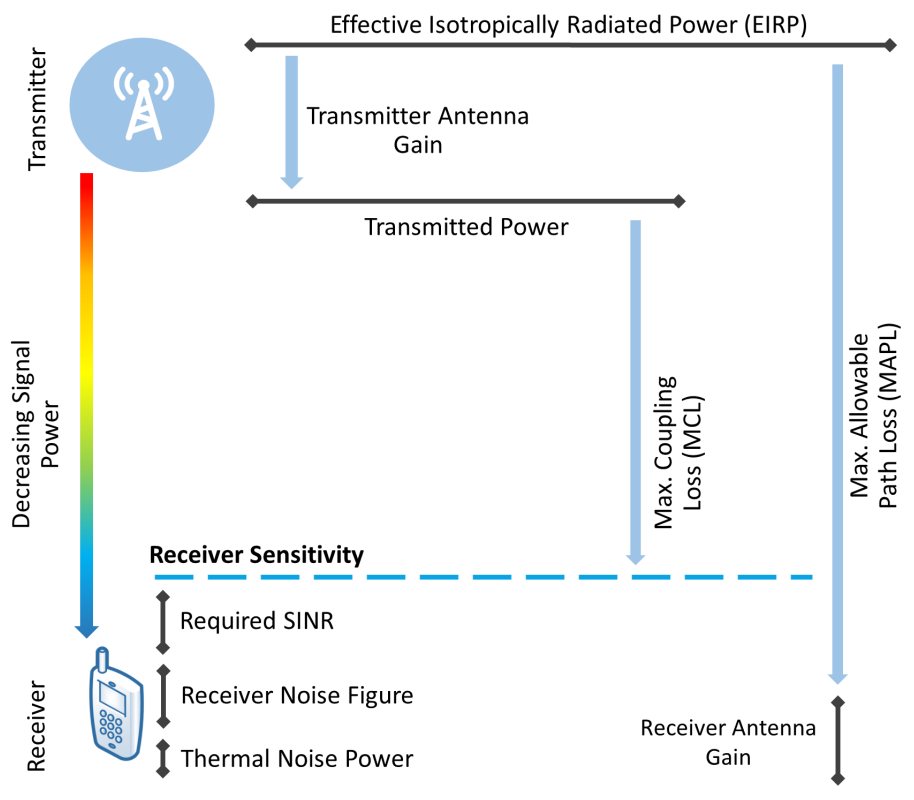

Figure 2. Maximum coupling loss and maximum allowable path loss system comparison (adapted from [43])
2) Scientific Literature: In literature, there are different ways to measure the network coverage, within different methods of network performance assessment. The outage probability is the most used coverage evaluation method. It is defined as the probability of a desired received signal power over the interference signal to be below a certain threshold. This outage probability can be measured regarding a specific threshold of a certain performance metric. The signal-to-noise Ratio (SNR) threshold when the interference is eliminated or not considered, or the signal-to-interference ratio (SIR) and SINR for dominant interference or noisy channels, respectively, are the most commonly used metrics [46].

Several researchers also discussed the coverage reliability concept, which can be separated into cell-edge reliability and cell area reliability [47], [40]. The former refers to the probability of the received signal quality to be above a certain threshold on a circular contour at the cell-edge. This is similar to the outage probabilities based on a SINR/SNR threshold. The latter aims to meet the same signal quality threshold after integrating the contour probability over the entire area of the cell. The authors of [40] propose an optimized metric for radio-frequency (RF) coverage quality classification. After showing that the more common metric based on the area 
reliability principle has some limitations for this classification, they complemented it with a cell radius inaccuracy criterion, obtaining an optimized coverage classification metric [40]. The authors not only discovered that the cell radius has an important impact in the cell coverage reliability estimation, but also they found that it is the main limiting factor. The cell radius inaccuracy study revealed that the number of real samples needed for a cell radius accurate estimation, is much bigger than for the cell area reliability. By accurately measuring the cell radius and combining those results in the area reliability equation, a better metric for coverage quality assessment is obtained. The authors of [48] also address a study on the cell radius and cell area coverage estimation by planning tools. By providing a method for the calculation of the distribution of the cell area errors, their work allowed to validate the coverage values predicted through network planning, within a certain confidence interval.

The SG is another widely used strategy in literature for network performance study. Despite the coverage being massively assessed through the outage probability in this technique, depending of each design, several conditions can be added to that metric. The SG provides a unified mathematical paradigm to model different types of networks, allowing to characterize their operation and behaviour [12]. Hence, the network coverage can be analysed through a spatial distribution of the SIR, which allows to measure the outage probability based on the SIR threshold. Such algorithms abandon the typical hexagonal grid which provides optimistic results. An irregular network topology which randomly changes from one geographical location to another is rather adopted. In [49], by using a SG, the authors assess the coverage probability in three different ways: (1) the probability that a randomly chosen user can achieve a target SINR, (2) the average fraction of users who, at any time, achieve a specific SINR value, and (3) the average fraction of the network area that is in coverage at any time. The coverage analysis on these three definitions shows that the SINR experienced by the users in a real BSs placement is upper bounded by the SINR of the users of the idealistic hexagonal grid. On the contrary, it is lower bounded by the SINR of the users in the random networks. In SGs, the standard approximation consists in calculating the transmission link through the complementary cumulative distribution of the SIR. Such calculations involve spatial averaging, i.e., the evaluation of a certain value in the Poisson point process. However, this method is considered limited since it only provides information on the success probabilities of the individual links, ignoring the users performance. As for the standard approximation, the Meta Distribution also considers calculating the distribution of the SIR, called success probability. However, it allows to get some insights on the network performance such as the discrepancies among the users performance, and the percentage of users experiencing a certain QoS [50],[51]. To achieve this, the Meta Distribution distributes the SIR as a result of the distribution of the conditional success probability. In this process the conditional probability is taken over the fading and the channel access scheme of the interferers, as in (3) [51]:

$$
P_{S}(\theta) \triangleq \mathbb{P}(S I R>\theta \mid \Phi)
$$

where $P_{S}$ is the success probability, $\mathbb{P}(x)$ represents the probability of $x, \theta$ is the desirable SIR threshold and $\Phi$ represents the $\mathrm{BS}$ Poisson point process, i.e., the BS random locations distribution in the area of interest for analysis. This approximation allows to answer questions such as "what fraction of users in a Poisson cellular network achieves 90\% link reliability if the required SIR is $5 \mathrm{~dB}$ ?" [51]. Still relying on SGs, in [52] and [53] the authors derive a new coverage definition taking into account the reliability thresholds for successful information data decoding and serving BS detection, as in (4):

$$
P_{c o v}\left(\gamma_{D}, \gamma_{A}\right)=P_{r}\left(S I R \geq \gamma_{D}, \overline{S N R} \geq \gamma_{A}\right)
$$

where $P_{\text {cov }}$ stands for coverage probability, $\gamma_{D}$ and $\gamma_{A}$ are the thresholds for information data decoding and BS detection success, respectively, $P_{r}$ is the received power probability and $\overline{S N R}$ is the average SNR. In this approach, the SNR, averaged with respect to the fast fading, is added to the coverage probability calculations. This allowed the authors to measure the weight of a UE detecting the pilot signal of at least one BS during the cell association process. Such a scenario reflects the tight interplay between the transmission power and BS density [53].

3) Industrial Forums: Other ways of measuring coverage can be more suitable for real scenarios. Ofcom, the U.K. spectrum regulator, provided a new metric for mobile coverage assessment based on the UE experience rather than focusing only on the projected signal quality [54]. This has been motivated by the fact that next generation mobile phones require stronger signals to fully operate, when compared to the older and simpler generations. Therefore, Ofcom separates the coverage definition in telephone calls and data services. A UE has good voice service coverage if it is able to complete a voice call during $90 \mathrm{~s}$ without any interruption. For data services, a user is within a coverage area if the data connection delivers at least 2 Mbps transmission rate, which allows to browse the internet and watch glitch-free videos. Such definition allowed to conclude that LTE voice calls and data coverage requires a higher signal level than previously estimated. Thus, an operator needs to deliver a minimum LTE signal strength of $-105 \mathrm{dBm}$, and a signal at least $10 \mathrm{~dB}$ higher than this to provide a similar good experience in indoor environments [54].

\section{B. Identification of Coverage Issues}

Mobile coverage represents one of the most challenging optimization parameters in mobile networks. It can be degraded by the increasing network density, in both users and network elements, as well as due to the environment morphology. Some of the main coverage issues in mobile networks are presented below. 
1) Coverage Holes: There are two main situations that can be classified as a coverage hole. Firstly, it can occur as a result of the pilot signal strength being below a threshold, which is required by a UE to access the network. Secondly, it may happen when the SINRs from both serving and neighbouring cells are below the minimum level to maintain a basic service. This situation can be caused by a bad network RF planning, unsuitable antenna configuration or due to the surrounding environment (e.g., obstructions, buildings and hills). The UE in these areas tends to suffer poor voice and data user experience, increasing both the probability of dropped calls and latency [55].

2) Weak Coverage: A specific area is considered with weak coverage when the pilot signal strength, or the SNR/SINR of the serving cell, is below the required level to maintain a defined performance. Nevertheless, contrary to the coverage holes, basic services or services working below the aimed performance, such as voice calls, could keep operating [55].

3) Pilot Pollution: Certain areas are considered under the pilot pollution phenomenon when there is an undesirable level of overlapping cells. This situation results in high interference, power levels and energy consumption. In addition, the cell performance tends to be low. In these locations, despite the received power may be significant, the UE experiences low SINR values due to the high interfering signals from different cells, which degrades the user QoE [55].

4) Overshoot Coverage: Overshooting happens when a cell coverage area reaches far beyond what is planned, creating an "island" of coverage in the inner region of another cell. This situation can be caused by successive signal reflections in buildings or across lakes and open water areas, resulting in call drops, high interference and bad QoE [55].

5) Channel Coverage Mismatch: Since the transmitted power is often much higher in the BSs than in the UE, a DL and UL channel coverage mismatch area may appear. Usually, users are connected with the BS that offers the best signal strength/quality in the DL. However, this may not always be the nearest BS, and hence, there could be another BS that offers better coverage in the UL. In other words, good coverage or the best serving BS in the DL does not guarantee good coverage or the best serving BS in the UL. Therefore, a UE in this mismatch area will suffer from UL performance degradation [55].

6) The Cell-Edge Problem: Most of the network coverage issues are concentrated in the boundaries of the cells serving area, the so-called cell-edge problem. The latter can be traced to the non-uniform distribution of the radio power in cellular systems. The received power of a radio signal decays exponentially with the propagation distance, which typically results in very significant path loss of the received signal for the users at the cell-edge [56]. Sectorization is an important solution to potentiate the network performance and, it is used in almost every BS configuration and deployment scenario. Increasing the number of sectors presents great benefits in terms of improved spectral efficiency, system capacity, and cell coverage extension by concentrating the transmitting power in the cell centre. The standard deployments consider 3 sector BSs, but this number can be higher, e.g., 6, 9 or 12 sectors [57],
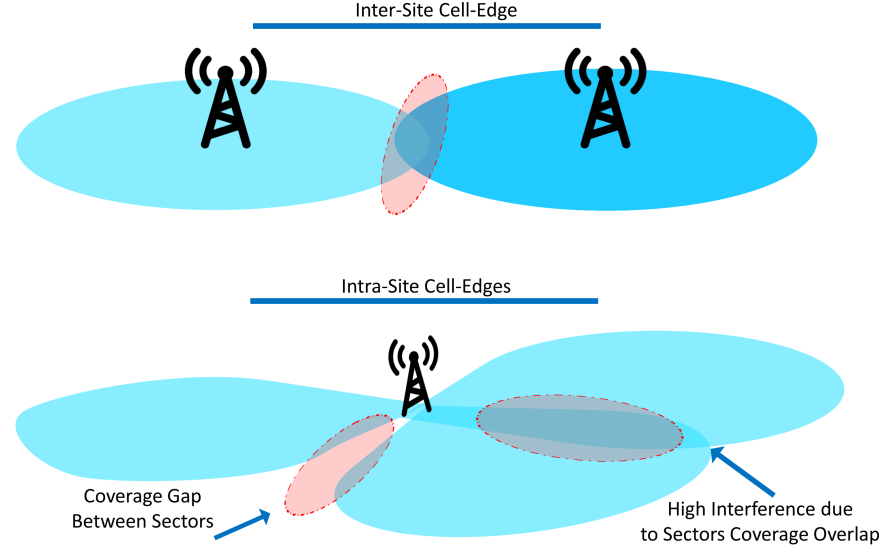

Figure 3. Cell-edge types in communication networks.

[58]. However, this configuration is directly related with some of the existing cell-edge problems. A general limitation of deploying the BSs in a multi-cell manner has to do with the stronger power transmission in the boresight direction of the cells. This results in a signal degradation not only linked with the distance to the cell, but also with the distance to the centre of the transmission main lobe. In addition, the edge problem is also highly related to the significant interference that the UE receives in the edge of the cell. This happens due to the overlapping signals within neighbouring BSs, which can be more intense due to the referred increased directivity. This overlap is also a problem within the sectors of the same BS. It can be caused by poor sector isolation, and due to more locations having a coverage overlap resulting from the signals transmitted by each sector, which will increase for a higher number of sectors. The coverage gap/hole or critical interference within the sectors of the same site is termed as the intra-site cell-edge problem. It represents another important cell-edge factor, which needs to be added to the more common inter-site issue.

The overall cell-edge problems can also be critical when moving to higher carrier frequencies, since the path loss exponent is larger and the transmission beams are narrower [56]. This might particularly aggravate the intra-site edge situation. Due to all these identified issues, a correct network planning which minimizes interference, and the appearance of new technologies for coverage improvement are of great importance. These factors become even more crucial taking into account the demanding requirements of future telecommunication networks. Therefore, most of the existing tools and techniques to overcome coverage limitations and challenges are explored in this article.

\section{Monitoring Network Coverage}

The aforementioned issues are correlated with most of the network coverage problems. Assessing and identifying these problems is of crucial importance for the correct operation of mobile networks. Identifying such events requires UE measurements through drive-tests (DTs), performance measurements, KPI analysis, alarms and traces [59]. The authors of [60] provide a self-diagnosis algorithm to detect 
coverage issues based on the aforementioned coverage holes, overshooting and pilot pollution scenarios. The assessment of these situations relies on periodically collected DTs or network traces. A reliability index is applied to these traces to quantify their significance. In this study, the reliability is measured through the computation of two indexes: the DT spatial distribution and the road filling ratio. These indexes measure how well can the field measurements characterize the study area, allowing reliable conclusions.

1) Performance Indicators for Coverage Assessment in LTE: In order to detect the coverage issues discussed in the previous section, some network KPIs provide a powerful data source for its assessment. Some of them, focused on the coverage holes detection, are listed below [61]:

- The Evolved Universal Terrestrial Radio Access Network Radio Access Bearer Retainability calculates the ratio of normally terminated connections over the total number of connections. This indicator tends to provide low ratios in areas with lack of LTE coverage.

- When a user starts to experience poor RF conditions in LTE, it sends an event-triggered measurement report to its serving cell, indicating that the Reference Signal Received Power (RSRP) is below a certain threshold. Counting the Number of Bad Coverage Reports (BCR) represents an important metric regarding coverage evaluation.

- The Handover Success Rate (HOSR) that provides the number of successful executed handovers.

- The Inter-RAT Handover rate (IRAT HO) indicates the percentage of the normal disconnections in LTE that were redirected to any legacy Radio Access Technology (RAT), namely $2 \mathrm{G}$ or $3 \mathrm{G}$. When the rates of this KPI are high, it could mean that there is a lack or weak LTE coverage.

2) Good Practices for Network Coverage Management: The common coverage issues can be mitigated by following good practices of network coverage management, as discussed in [62]. For example:

- Establishing a coverage mapping of the entire network provides a broader view of the network coverage, which allows to assess the signal levels that can be achieved in each location. This requires measurement collection from the whole network, instead of focusing only in the areas with potential coverage issues.

- The poor UL coverage may result in call setup failure, call drop and degrades the service quality. Therefore, to balance both UL and DL connection, coverage is crucial in good planning principles.

- A cell boundary mapping allows to identify the cell boundaries and the physical conditions of the surrounding areas. It provides a powerful method to manage coverage performance.

- The KPI analysis based on network performance management (PM) data provides key parameters to identify coverage issues.

3) Three-Dimensional Network Coverage: Network coverage management and planning in legacy mobile technologies is often conducted via a two-dimensional (2D) coverage analysis, i.e., coverage area captured by metrics such as bits $/ \mathrm{s} / \mathrm{m}^{2}$. Nonetheless, a three-dimensional (3D) coverage network assessment is increasingly becoming more important. The 2D coverage planning does not properly consider blockages and environment obstacles which can lead to unrealistic and, usually, optimistic results [63]. This is essential when planning in urban areas with high-rise buildings, future mm-Wave deployments taking into account its propagation limitations. Similar issues can be found for directed beams, such as in beamforming solutions. In these scenarios, not only is it important to consider the horizontal beam, but also the vertical component when UEs in tall buildings are being served. A 3D geographical environment includes the coordinates of buildings and their heights from the ground [64]. In addition, new solutions for coverage improvement are no longer restricted to terrestrial infrastructure. UAVs and satellites are increasingly being merged with telecommunication networks. Assessing its performance requires a 3D spatial view. The same can be said for new solutions making use of spatial multiplexing. Therefore, coverage evaluation metrics should evolve from typical throughput or spectral efficiency per square meter to throughput per cubic meter. The 3GPP has already defined 3D antenna radiation patterns and path loss models [65], [66]. With the evolution of computational processing capacity, the implementation and use of these models is significantly more viable than ever before. Therefore, the evaluation and design of new cellular technologies can be more adapted to the real network behaviour.

\section{Network Planning Tools}

Network planning tools play an important role in predicting performance of mobile networks. There are several available tools, and some of the most famous are briefly explored here. The ASSET radio planning tool from TEOCO [67] represents one of the most famous tools for network planning, with features related to network coverage, cell parametrization and neighbouring planning. It is compatible with all mobile technologies, including 5G, and contains high resolution maps for reliable coverage prediction [67]. The MapInfo tool [68] is also widely used in the industry for network planning/troubleshooting. It works with positioned data allowing to plot the network coverage in a map view [68]. The Atoll planning tool from Huawei [69] has integrated capabilities for network design and optimization across all mobile technologies. It can be used with live network data, based on KPIs or traces, to model and predict future network states. In addition, it includes a high performance geographic information system, allowing to work with high-resolution and large-scale geo-spatial data [69]. Other tools such as the RF-Planning software from InfoVista [70] or Ranplan from O-RAN Alliance [71] also bring interesting features for network coverage planning. The former operates from the third generation of mobile communications, integrating both geo-spatial data and the test mobile system (TEMS) network testing portfolio [70], [72]. The latter provides a solution encompassing both indoor and outdoor mobile service radio 
planning [71].

Achieving performance targets of cellular systems may require following some good practices. The planning tools should be used and complemented with optimization of network configuration, such as transmit power, antenna tilt, and azimuth, coupled with network deployment and interference mitigation techniques. A detailed discussion on the existing coverage enhancement mechanisms is presented in Sections IV, V and VI. Before that, it is important to explore some of the main requirements and KPIs regarding mobile telecommunication technologies, highlighting how coverage relates to them. Establishing this relationship between network KPIs and coverage is important to understand and measure the impact of coverage quality in the network performance. Therefore, the following section goes through some of these main KPIs, for both LTE and 5G, focusing on how providing good coverage can help to achieve the QoS targets.

\section{Coverage And Mobile Network Requirements}

Across all legacy mobile communication technologies, several requirements were defined before their initial releases. The same is happening for $5 \mathrm{G}$, with ambitious and challenging performance requirements defined, mainly for capacity, latency and massive connectivity [73], [74]. This section highlights some of these requirements and KPIs, focusing on the LTE and the 5G. The link between these KPIs and network coverage is shown, and quantifies the benefits of coverage enhancement in achieving the target performance figures.

\section{A. Performance Requirements for Coverage in Cellular Net- works}

1) LTE and LTE-A: According to LTE Release 9 [75], depending on the cell range, coverage should be provided to meet the throughput, spectral efficiency and mobility targets. These requirements were updated in the further LTE-Advanced (LTE-A) releases [76], bringing new enhancements to the technology with the introduction of new concepts and more ambitious requirements. The network coverage requirements follow this evolution and some of the goals for LTE-A are presented in Table II.

Table II

LTE-A Coverage ReQuirements [76], [16].

\begin{tabular}{|c|c|c|}
\hline \multicolumn{2}{|l|}{ KPI } & Requirements \\
\hline \multirow{7}{*}{ Cell-Edge User Throughput } & Configuration & $\begin{array}{c}\text { DL } \\
{[\mathrm{bps} / \mathrm{Hz} / \text { cell/user }]}\end{array}$ \\
\hline & $2 \times 2$ & 0.07 \\
\hline & $4 \times 2$ & 0.09 \\
\hline & $4 \times 4$ & 0.12 \\
\hline & Configuration & $\begin{array}{c}\text { UL } \\
\text { [bps/Hz/cell/user] }\end{array}$ \\
\hline & $1 \times 2$ & 0.04 \\
\hline & $2 \times 4$ & 0.07 \\
\hline $\begin{array}{l}\text { Maximum Supported } \\
\text { Coupling Loss }\end{array}$ & \multicolumn{2}{|c|}{$140 \mathrm{~dB}$} \\
\hline
\end{tabular}

The LTE-A requirements in Table II vary with the cell range and mobility. In cell ranges up to $5 \mathrm{~km}$ all targets should be met, while for ranges up to $30 \mathrm{~km}$, some degradation on throughput and spectral efficiency is acceptable. In the $\mathrm{DL}$, the $5^{t h}$-percentile of the UE throughput per MHz should be 2-3 times higher than the High-Speed Downlink Packet Access (HSDPA) Release 6 [77]. In the UL scenario, both UL and DL targets establish an improvement of 2-3 times the Release 6 Enhanced Uplink [78]. It matters to highlight that, for both scenarios, such requirements are limited to the use of 2 transmitting and receiving antennas. For the spectral efficiency, the DL target in bps/Hz/site is 3-4 times higher than the HSDPA Release 6, and the UL target is 2-3 times the Enhanced Uplink Release 6. The latter target is assumed for a single transmission antenna [75].

2) $5 G$ New Radio: In 5G, three main use cases are introduced: the enhanced Mobile Broadband (eMBB), representing the data-driven use cases which require high data rates, the mMTC, that stands for the need to support a massive number of devices in a small area, and Ultra-Reliable and Low-Latency Communications (URLLC), for mission critical communications. The main developments in $5 \mathrm{G}$, including coverage enhancement solutions, are aligned with the established objectives for these use cases. The $5 \mathrm{G}$ performance requirements are detailed in [73], [74], [79] and [80].

Table III presents the 5G KPIs related to coverage. Additionally, to ensure service everywhere, a 5G system should be able to reduce data transfer rate at the cell-edge for very large coverage areas, e.g., limiting $100 \mathrm{kbps}$ for a cell radius larger than $100 \mathrm{~km}$, or $1 \mathrm{Mbps}$ for cell range of $100 \mathrm{~km}$ [81]. The concept of network access everywhere will also demand long range coverage in low density areas such as extreme rural or even at the sea. In such scenarios, the system should support long-range coverage up to $100 \mathrm{~km}$ in low density areas, with up to 2 users per $\mathrm{km}^{2}$. The minimum throughput at the celledge in such scenarios is defined as $1 \mathrm{Mbps}$ for DL and 100 kbps in UL. The End-to-End (E2E) latency for voice services at the edge of coverage should be less than $400 \mathrm{~ms}$.

3) $5 G$ Backbone: Another important factor to enable $5 \mathrm{G}$ coverage is related to the requirements regarding the backbone/core and backhaul network availability. In order to enable efficient $5 \mathrm{G}$ coverage, it is critical to enhance the core network and provide high capacity links at the backhaul, allowing to deploy new infrastructure at almost any location. This must be aligned with requirements such as less than $1 \mathrm{~ms}$ latency across the backhaul network, support for 1000 times more capacity generated by $5 \mathrm{G}$ networks, and handling the expected 3.5 million additional connections resulting from dense small-cell deployments [82]. Therefore, optimization of the backbone architecture is imperative. This can be accomplished by keeping a scalable approach, defining more efficiently the required number of backbone nodes for a certain target performance [83]. The deployment of high capacity links, usually by means of optical-fiber, and algorithms for management of increasing link density together with optimized routing for information transmission are also required. In addition, creating mechanisms of redundancy and resiliency in the backbone network to face potential node operating issues, maintenance, or disaster recovery are challenges to be addressed [84]. The study of alternative backbone networks, 
such as non-terrestrial backbone network based on satellites or UAVs for enabling coverage in rural and isolated areas needs to be considered [85]. Moreover, the development of slicing and virtualization components, as well as the capacity to operate with software-defined nodes and functionalities, constitute requirements and potential directions of future cellular backbone technologies [86].

\section{B. Key Performance Indicators Related to Coverage}

Coverage enhancement mechanisms can positively impact several network KPIs. In this section, the coverage-KPI relation is explored, highlighting mainly the $5 \mathrm{G}$ network KPIs presented in Table III, and how coverage deployments can enhance them. This in turns allows to understand the importance of good coverage when aiming high network performance.

1) Coverage Effect on User Experienced Data Rate and Spectral Efficiency: These two KPIs can be grouped together since they are related with each other. The user experienced data rate is similar to the tail perceived throughput discussed in Section II-A. It can be computed as the product of the channel bandwidth and the $5^{t h}$-percentile user spectral efficiency [74]. The spectral efficiency is a function of the SINR. Higher modulation schemes can be used as the SINR increases which allows transmitting more bits/Hz. Good coverage is one of the most important factors for SINR quality improvement at the cell-edge. For instance, the study in [88], where a 4 relay node solution is tested, shows that the SINR gains in the cell-edge increase approximately $100 \%$ (3 dB) per physical resource block in non-interference-limited suburban scenarios. Consequently, a gain of approximately $120 \%$ is observed in the $5^{\text {th }}$-percentile user throughput. This happens taking into account that coverage enhancement solutions do not simply increase the signal power at the cell boundaries. Essentially, they need to assure that the increase in the desired signal is higher than the existing interference at the cell-edge. Thus, solutions which aim to enhance coverage are expected to positively affect the users at the cell-edge and hence, improving the $5^{t h}$-percentile SINR and spectral efficiency.

The LTE study reported in [13], where real LTE measurements are compared with simulations, supports the dependency between DL throughput and SINR in both measured and simulated data. These considerations take into account the coverage definition based on the SNR/SINR thresholds. Additionally, the same LTE performance study [13] also addresses the relationship between path loss and SINR. It shows a linear common behaviour within these two metrics when the LTE network is lightly loaded. This result creates a connection between the MAPL metric (used to define coverage) and the DL throughput. Therefore, solutions for coverage enhancement with positive impact in both MAPL and SINR can improve the $5^{\text {th }}$-percentile network data rates. Solutions with impact in the SINR or path loss such as network densification that decrease the distance between the APs and the end-users, or taller masts for diffraction loss mitigation, are at the same time increasing the network throughput and spectral efficiency.

The study in [88] also shows further improvements in the $5^{\text {th }}$-percentile throughput when 10 relay nodes are used in urban environments. An increment of $210 \%$ edge throughput was verified when the relay node solution was merged with power control and biased mechanisms. This translates into $5^{\text {th }}$-percentile throughput nominal values of $200-350 \mathrm{kbps}$, compared to the $100 \mathrm{kbps}$ achieved with the eNB only. Similar results are provided for the UL scenario. This study can be considered for improvement of the LTE-A requirements from Table II. The minimum cell-edge spectral efficiency is defined as $0.07 \mathrm{bps} / \mathrm{Hz} /$ user. Assuming a system bandwidth of 10 $\mathrm{MHz}$, this minimum requirement is equivalent to $700 \mathrm{kbps}$. Following the presented results of [88], increasing the $5^{\text {th }}$ percentile LTE-A minimum rate value by $210 \%$ would result in $2170 \mathrm{kbps}$, i.e., $0.217 \mathrm{bps} / \mathrm{Hz}$. This value becomes close to the $5 \mathrm{G}$ requirement stated in Table III. It is interesting to recall that these results are achievable by only optimizing the cell-edge performance with relays, power control and biased values. Additional capacity deployments can be added with small cells or diversity (the study only considers a $2 \times 2$ antenna configuration), which possibly result in even higher gains. Some of the further highlighted studies in this paper, mainly in Section IV-A, address the increase in SINR and spectral efficiency as a function of the BSs density [89], [90]. The analysis in [90], following the 3GPP recommended parameters for small cell networks, shows that with a BS density of 20 $\mathrm{BS} / \mathrm{km}^{2}$, the coverage probability defined by a SINR threshold of $3 \mathrm{~dB}$ increases up to $60 \%$. If this threshold is decreased to $0 \mathrm{~dB}$, the coverage probability reaches $80 \%$.

Another interesting relationship with the $5^{\text {th }}$-percentile and average spectral efficiency KPIs can be established with the SINR and bit error rate (BER)/BLER metrics, since these KPIs depend on the number of correctly received bits per user. The BER/BLER metrics decrease as the network coverage improves, i.e., with higher SNR/SINR ratios, since they contribute towards a more robust and reliable transmission. The study in [13] also provides a relationship between SINR, path loss and the channel quality indicator (CQI). The latter is shown to be higher for better SINR and lower path loss. Therefore, improving the SINR/SNR enhances the CQI which allows using higher modulation coding schemes. This in turns allows to transmit more bits for the same BER/BLER constraint. Beamforming is considered one of the main enabling technologies for solutions based on mm-Wave and massive MIMO. This technique improves the coverage range of these solutions by directing the beams towards the UE. In [30], the authors show that a solution based on mm-Wave and adaptive beamforming, operating in a $28 \mathrm{GHz}$ carrier configuration, can achieve a BLER below $1 \%$ in a 16-quadrature amplitude modulation (QAM) scheme for distances up to $1.7 \mathrm{~km}$ from the base station. This translates into $528 \mathrm{Mbps}$ data rate per user compared to $264 \mathrm{Mbps}$ achievable with a quadrature phase-shift keying (QPSK) modulation scheme to be used when the BLER constraint is not fulfilled. This provides an example where improving coverage in high capacity solutions can duplicate the number of correctly transmitted bits for the same bandwidth.

2) Coverage Effect on Connection Density: The connection density KPI envisions mainly the mMTC use case where millions of devices are connected within relatively small areas. 
Table III

5G REQUIREMENTS RELATED TO COVERAGE [74], [87].

\begin{tabular}{|c|c|c|c|c|}
\hline KPI & \multicolumn{3}{|c|}{ Requirement } & Considerations \\
\hline $\begin{array}{l}\text { User Experienced } \\
\text { Data Rate }\end{array}$ & \multicolumn{3}{|c|}{$\begin{array}{l}\text { DL: } 100 \mathrm{Mbps} \\
\text { UL: } 50 \mathrm{Mbps}\end{array}$} & $\begin{array}{l}5 \% \text { CDF of the eMBB } \\
\text { user throughput }\end{array}$ \\
\hline \multirow{4}{*}{$\begin{array}{l}\text { 5th Percentile User } \\
\text { Spectral Efficiency }\end{array}$} & Environment & $\begin{array}{c}\text { DL } \\
\text { (bps/Hz) }\end{array}$ & $\begin{array}{c}\mathbf{U L} \\
(\mathbf{b p s} / \mathbf{H z})\end{array}$ & \multirow{4}{*}{$\begin{array}{l}5 \% \mathrm{CDF} \text { of the normalized eMBB } \\
\text { user throughput. }\end{array}$} \\
\hline & Indoor Hotspot & 0.3 & 0.21 & \\
\hline & Dense Urban & 0.225 & 0.15 & \\
\hline & Rural & 0.12 & 0.045 & \\
\hline Connection Density & \multicolumn{3}{|c|}{1 Million Devices per km2 } & For mMTC \\
\hline \multirow{5}{*}{ Mobility } & Environment & $\begin{array}{c}\text { DL } \\
\text { (bps/Hz) }\end{array}$ & $\mathbf{k m} / \mathbf{h}$ & \multirow{5}{*}{$\begin{array}{l}\text { Maximum eMBB mobile speed at which } \\
\text { a defined QoS can be achieved. }\end{array}$} \\
\hline & Indoor Hotspot & 1.5 & 10 & \\
\hline & Dense Urban & 1.12 & 30 & \\
\hline & Rural & 0.8 & 120 & \\
\hline & $\begin{array}{l}\text { High Speed Vehicular } \\
\text { (Train) }\end{array}$ & 0.45 & 500 & \\
\hline $\begin{array}{l}\text { Supported Maximum } \\
\text { Coupling Loss }\end{array}$ & \multicolumn{3}{|c|}{$164 \mathrm{~dB}$} & \multirow{3}{*}{$\begin{array}{l}\text { Considering deep indoor } \\
\text { mMTC/IoT scenarios. }\end{array}$} \\
\hline Data Rate at the MCL & \multirow{2}{*}{\multicolumn{3}{|c|}{$\frac{160 \mathrm{bps}}{10 \mathrm{~s}}$}} & \\
\hline Message latency at the MCL & & & & \\
\hline
\end{tabular}

It is defined as the total number of devices fulfilling a specific QoS per unit area [74]. Coverage enhancement solutions can have a significant impact in these type of communications. This impact is not measured in the capacity dimension but rather by enabling this connectivity, e.g., towards the IoT concept where low power and light traffic devices are considered. Providing wide coverage solutions such as in rural environments for agriculture, or using lower frequencies to achieve high indoor penetration, are essential scenarios for future networks. The new LTE-Machine Type Communication (LTE-M) and Narrowband-IoT (NB-IoT) 3GPP standards, with increased MCL of $156 \mathrm{~dB}$ and $160 \mathrm{~dB}$ respectively, operating in low bandwidth carriers envision this use case [16]. The study in [91], for a rural area machine-type communication (MTC) testing scenario, shows that it is possible to achieve a deep indoor coverage (30 dB penetration loss) in the order of $80 \%$ and $95 \%$ with the LTE-M and NB-IoT, respectively. A more detailed view on these standards is provided in section $\mathrm{V}$.

3) Coverage Effect on Mobility: The mobility KPI refers to the maximum UE speed at which a defined QoS can be achieved [74]. It has been an important concern for mobile networks and tightly linked with the seamless coverage requirement. The latter demands a minimal QoS and QoE for all users at any environment or mobility scenario. Coverage in mobility becomes more critical when a significant number of connected and autonomous vehicles depend on the cellular networks for normal operation [92]. The German 5G spectrum auctions introduced for the first time coverage obligations regarding roads, rails and waterway routes. These obligations include a requirement of $100 \%$ coverage in motorways in 2022, and a minimum of 50 Mbps data rate when covering small roads, railways, seaports and the main waterways [93]. Coverage enhancement deployments such as cell range expansion, extremely long range communications, achievable with low frequency carriers, or even beamforming allowing to concentrate most of the transmitted power in a well defined direction, are solutions that can conduct to this seamless coverage. In addition, these solutions can minimize the handover rate by increasing the cell range rather than deploying new APs.

4) Data Rate and Latency at the Maximum Coupling Loss: According to 3GPP, coverage requirements are proposed to enable the three main 5G use cases [80]. For the mMTC use case, the coverage target is limited to a MCL of 164 $\mathrm{dB}$ assuming a $160 \mathrm{bps}$ service. For basic eMBB services characterized by data rates of $2 \mathrm{Mbps} / 60 \mathrm{kbps}$ for DL and UL stationary users respectively, 3GPP proposed a MCL target of $140 \mathrm{~dB}$. When a basic eMBB service is characterized by a DL and UL data rates of $1 \mathrm{Mbps}$ and $30 \mathrm{kbps}$ respectively, the target MCL is $143 \mathrm{~dB}$. For the mobile users, a DL data rate of $384 \mathrm{kbps}$ is acceptable, for the same MCL requirements. At the MCL target of $164 \mathrm{~dB}$, the latency should be $\leq 10$ s [80]. These KPIs are directly linked with coverage, since they are defined at the MCL which is one of the coverage metrics defined by the $3 \mathrm{GPP}$.

This section provided an overview on the main cellular network requirements. It establishes a connection between them and coverage, showing the importance of the latter for a proper and optimized network performance. Several mechanisms are being studied to meet all these performance targets. Nevertheless, the requirements related to coverage, latency and capacity represent a critical trade-off in mobile networks. For example, the LTE link budget study in [8] found that the uplink control channel (PUCCH) transmitted during a $1 \mathrm{~ms}$ time-interval has a coverage range of $1.4 \mathrm{~km}$ and 1 $\mathrm{km}$ for suburban and dense urban non-line-of-sight (NLoS) environments, respectively. These results are obtained for four receive antennas at the eNB, assuming a carrier frequency of $2 \mathrm{GHz}$. Reducing this transmission time to $0.2 \mathrm{~ms}$, as assumed in 5G small cell scenarios, is estimated to reduce the coverage range to $300 \mathrm{~m}$. In mm-Wave, a high system capacity increase is expected. However, these frequency bands have significant issues due to the high propagation loss and sensitivity to obstacles, decreasing the cell coverage area. Interference mechanisms can overcome some problems at the cell-edge. On the other hand, some of the mechanisms are 
complex and their processing time may affect the latency targets. The coverage can be significantly improved with lower frequency deployments using the 700-800 MHz carriers. Nevertheless, this is a limiting factor for capacity.

In the following sections, most of the existing mechanisms for coverage enhancement will be discussed. These solutions are expected to enhance the network performance and meet the performance requirements of the KPI of interest. The advantages of each mechanism are explored together with important limitations with regards to the latency-capacity-coverage tradeoff. A new taxonomy is defined for the coverage enhancement as follows:

- Network deployments: this encompasses most of the available and projected physical deployments of network elements for the coverage enhancement, such as aerial platforms, small/femto cells, relays, sectorization or reflectors.

- Frequency bands: this addresses the influence of different frequency bands on network coverage enabling very long range to short range and indoors coverage.

- Interference management: interference is expected to increase in telecommunication networks due to the growing number of connected devices and transmitting network elements. Therefore, the last of these three sections addresses the impact of different mechanisms for interference mitigation on coverage quality.

\section{Network Deployments for Coverage ENHANCEMENT}

Several coverage improvement mechanisms have been proposed and implemented. Most of these mechanisms focus on network deployment. The techniques in this category depend on new physical solutions, elements or instruments which can be added to the existing network infrastructure. An overview of such mechanisms is presented in this section. We evaluate the main contributions, limitations and challenges. It is worth mentioning that the coverage enhancements explored in this and in the following sections are not limited to extending the coverage radius. In some scenarios, increasing the coverage radius may result in additional network interference. In this direction, whether the coverage radius is augmented or not, the assessed results are considered as coverage enhancements based on the coverage definitions previously provided. This means that the gains in the desired signal are higher than the increased interference from neighbouring BSs.

\section{A. Densification and Heterogeneous Networks}

Providing wide and reliable network coverage is essential for future scenarios of cellular networks, as discussed in the previous sections. Network densification is one of the possible solutions to achieve these goals. It focuses on the deployment of cellular networks with massive addition of small cells, femto cells or relays, enabling D2D and Machine-to-Machine communications, in multi-tier HetNets. The latter represent a particular case of densification, aiming to connect different AP types, including macro, small, pico and femto cells as well as different RATs. These solutions allow to offload the macro cell traffic to small cells [94], providing coverage in the tens of meters, especially for indoor hotspots and dense urban micro cells. Since more APs are being deployed, they can be strategically placed near the end-user, improving the data rates, enabling low-latency applications, and increasing the coverage quality [17], [95].

In order to better understand the gains with network densification, the authors of [18] evaluated the results of diminishing the $200 \mathrm{~m}$ inter-site distance from a standard configuration to $35 \mathrm{~m}$. The results show an average UE throughput increase of 7.56 times for $100 \mathrm{MHz}$ bandwidth and up to $1.27 \mathrm{Gbps}$ for $500 \mathrm{MHz}$ bandwidth, considering a UE distribution of 300 active users per $\mathrm{km}^{2}$. Furthermore, in tests for extreme densification with inter-site distance of $5 \mathrm{~m}$, enhancements of 48 times for the cell-edge UE throughput were achieved. This solution, together with high frequencies, was found to be better in terms of network performance when compared with beamforming, which achieved average gains of $30 \%$ and 2 times better throughput at the cell-edge, in a 4 antennas per small cell configuration. Nevertheless, some of these gains seem to exist only until a certain BSs density threshold [95]. In [96], a study on mobility and coverage in HetNets is performed, with the BS location being modeled by the Poisson point process SG, with SIR distribution for coverage probability assessment. Despite results in capacity keep following the usual increase with densification, the probability of coverage is shown to be independent of the AP density. Moreover, if moving users are considered, this coverage probability becomes worse with densification due to a handoff rate increase as a result of smaller coverage areas provided by each BS. The authors of [96] report that the handoff rate increases with network densification, comparing a uniform user distribution with users performing a radial movement away from the serving BS. It is shown that from $1 \mathrm{AP} / \mathrm{km}^{2}$ to $10 \mathrm{AP} / \mathrm{km}^{2}$, when users are moving at an average speed of $5 \mathrm{~m} / \mathrm{s}$, the handoff rate can increase by $30 \%$ and $50 \%$ in the uniform and radial UE distributions, respectively. Regarding the network performance, the same conclusion was drawn in [49], since the increased signal power is counter-balanced by the increase in the interference power. In [19], by using the Poisson point process, it is concluded that the relationship between densification and throughput is not linear. Increasing the number of cells increases the inter-site and inter-cell interference. As the number of cells massively increase, the BSs are closer to the end-user, which means more line-of-sight (LoS) situations between the transmitters and the receivers. The authors of [19] also analyse the BS density threshold where the system spatial throughput, defined as the success probability of data transmissions at a certain SINR threshold, starts to degrade. SINR thresholds of $10 \mathrm{~dB}$, $15 \mathrm{~dB}$, and $20 \mathrm{~dB}$, and a path loss exponent of $\alpha=3.5$ in urban environments were considered. The spatial throughput starts to decrease when the BS density reaches approximately $6.31 * 10^{4} \mathrm{BS} / \mathrm{km}^{2}, 3.16 * 10^{3.5} \mathrm{BS} / \mathrm{km}^{2}$, and $1.58 * 10^{4}$ $\mathrm{BS} / \mathrm{km}^{2}$ for each SINR threshold, respectively. Taking into account the 1 million connections per square kilometer target, the best case scenario came up with a requirement of 16 connections/BS. The same conclusion was drawn when using a sophisticated path loss model that incorporates both LoS and 
NLoS transmissions. Based on this, several studies show that the interference overcomes the desired signal power due to the increasing LoS components as a result of densification [89], [90], [95], [97]. This means that the interferer sources will not only increase, but will be more powerful as well. Therefore, after a certain threshold of $\mathrm{BSs} / \mathrm{km}^{2}$, densification can not only prejudices coverage, but also the overall network performance.

However, according to [98], this situation is not verified when the BSs outnumber the mobiles. In this scenario, for a fixed UE density, increasing the number of BSs does not change the interference experienced by the user, but the signal power increases due to proximity to the BSs. Hence, the outage probability decreases for higher BS density. Furthermore, in [99], the deployment of BSs with idle-mode capabilities allows to activate BSs based on the users density, which may decrease the inter-site interference. Nevertheless, for high user density that requires more active $\mathrm{BSs}$, the same performance drop is noticed.

In general terms, there are other concerns regarding densification. The deployment costs for such a high number of BSs are anticipated to be very high. This become more critical in an era where operators and vendors are trying to offer to end-users more data applications, bigger data packages and unlimited phone calls without increasing the costs. Additionally, there is the energy efficiency problem. Despite the fact that small cells energy consumption is less than macro-cells, the number of deployed small BSs is very large which could result in a dramatic increase in the overall network energy consumption. Another important issue may rely on the network planning area. Most of the propagation models are formulated for NLoS scenarios while, due to the high number of BSs in a densified network, most users are expected to be served on a LoS basis [18].

\section{B. Cell Range Expansion}

In addition to improving the overall network capacity, densification and HetNets aim to increase the cell-edge performance as well as extending the coverage area provided by macrocells. By deploying different network tiers, HetNets offer wireless coverage in environments with extensive diversity, ranging from indoor to outdoor [37]. In the following subsections several mechanisms with cell range extension capabilities are explored.

1) Relay Nodes: Relays are defined as nodes with compact physical characteristics, low power consumption and flexible deployment, which are targeted for coverage extension and load balancing [88]. Such deployments bring important advantages for the cell-edge UE, since introducing relays in edge locations significantly increases the SINR experienced by the users. The same can be stated for coverage holes. The authors of [100] study the benefits of cooperative diversity in heterogeneous relay networks in terms of outage probability for 5 different relay scenarios. Closed-form expressions for outage probability were derived for homogeneous networks (where all terminals are located in the same environment, i.e., indoor or outdoor), heterogeneous source, heterogeneous destination and homogeneous relays (with all relays located in the same environment). The results show improvements in the outage probability, mainly in the homogeneous indoor scenario.

Nevertheless, sharing some of the densification challenges, there are some inherent adversities to the relays. These include inter-cell interference and misleading in UE cell connection and handover due to the small transmission power of the relays compared to the macro cells. Hence, mechanisms for inter-cell interference coordination (ICIC), relays deployment planning and macro cell power control need to be exploited to overcome these challenges. Taking these limiting factors into account, a joint optimization of eNB power reduction, biasing value and number of backhaul subframes in urban scenario was developed in [88]. The $5^{t h}$-percentile UE throughput was optimized and improved by $200 \%$. Similar results were verified in [101], with the addition of optimized handover thresholds. A coverage extension around $300 \mathrm{~m}$ and cell-edge users throughput with a minimum data rate of $20 \mathrm{Mbps}$ were also achieved in studies under a Berlin testbed [102], where a relay node was deployed in a van and placed at the celledge of a BS sector [103]. In [104], with the objective of coverage region maximization, the authors focus on a relay selection technique for cooperative cellular networks, with multiple fixed and unfixed relays. The fixed relays are the relay nodes installed at optimal locations, while the moving relays are network users which can act as relays, in a similar concept to the D2D communications.

2) Small, Femto and Pico Cells: With common objectives, the small cells can represent a particular scenario of relays. However, since these elements have their own backhaul links, they improve both the coverage and the capacity. In [105], by deploying a small cell in a moving bus, a coverage extension up to $300 \mathrm{~m}$ in an urban environment was obtained. Small cells encompass mainly femto-, pico- and micro-cells. Their maximum covering area radius is proportional to their transmit power, with $35 \mathrm{~m}$ for femtocells, $200 \mathrm{~m}$ for picocells and $2 \mathrm{~km}$ for microcells [106]. As aforementioned, the newly admitted users in relay nodes increase the resource demand of the relay on the wireless backhaul link from its eNB. On the contrary, the small cells can be deployed with dedicated backhaul links that allow offloading macro-cell users.

The femtocells are low-power and subscriber/operator independent BSs. They provide a cost-effective solution to significantly improve indoor coverage and offload traffic from the macro-cells network. They typically cover a range up to $50 \mathrm{~m} \mathrm{[37],} \mathrm{[106].} \mathrm{By} \mathrm{offloading} \mathrm{the} \mathrm{macro-cell} \mathrm{traffic,}$ femtocells can also improve the network average throughput [107]. Projected mainly for indoor use, the femtocells can be deployed near the end-user, with a short distance between the transmitter and receiver, and they are usually connected to the core network. The femto-cells provide a direct channel access, with minimal delays and high SINR, which represent an important solution for edge UEs [37]. Due to the low transmission power, up to $200 \mathrm{~mW}$, femtocells provide a solution for initial densification, with minimal additional interference, mainly when located indoor. On the other hand, this low transmit power demands planning and optimized 
deployment, depending on the environment. According to [108], the walls type, the distance between a UE and the femtocell as well as the number of walls between them, can highly affect the performance of this technology. The outage probability can increase up to $40 \%$ for some wall structures, up to $80 \%$ when the users are $25 \mathrm{~m}$ away, and up to $90 \%$ when there are at least 5 walls between the femtocell and the user. Interference management will have an important role in future massive femtocell densification, not only due to interfemtocell interference but because they are deployed in the same spectrum as the legacy (outdoor) wireless networks. The mobility and seamless handover operations also need to be correctly managed, otherwise the communications will be interrupted due to the small covering area of these network elements [106], [109].

With cell radius up to $200 \mathrm{~m}$ and transmission power of $250 \mathrm{~mW}$, picocells support more users than the femtocells, being ideally deployed in wide indoor areas or outdoor [110]. This small cell type shares some of the main benefits of femtocells, such as coverage expansion, proximity to end-users and coverage holes mitigation, but is projected for slightly wider areas. Due to its higher transmission power, picocells can increase the cell range up to $40 \%$ [111]. Since the majority of the picocells are deployed outdoor, under the coverage provided by the macro cell but with a much lower transmission power, the same UE connection problems faced by the relays tend to happen. In more detail, due to the transmitted DL power difference between the macro and picocells, the area where the picocell is dominant in terms of DL power becomes smaller as the picocell is deployed near the macro BS. Thus, the users may connect to a distant high-power macro BS rather than the nearby picocells, which prevents the desirable efficient load balance. This situation can also bring increasing problems regarding handover boundaries [112]. The cell association based on the strongest DL received signal strength (RSS) may not be the best strategy for this scenario. Hence, mechanisms for cell association and ICIC that increase the SINR of this low power network elements when operating under an umbrella coverage provided by a macro cell, need to be addressed [111], [112].

3) Device-to-Device Communications: Another popular emerging technology, which is also considered as a promising coverage extension technique for cellular networks, regards on the D2D communications [81]. D2D communications have emerged as a technology that apart from the benefits of acting as network relays, can offload the macro cells signalling by certain network procedures being performed independently within the UE. This technology takes advantage of the proximity of communicating devices for efficient utilization of available resources, reducing latency, improving system capacity and spectral efficiency. In D2D communications, the spectrum resources can be shared in an inband or outband category. In the former, D2D communications use the licensed spectrum, while the latter uses the unlicensed spectrum. The D2D inband operation can also be divided into underlay and overlay communication. In the underlay scenario, the D2D users share the same frequency resources as the cellular users.
In overlay communications, the cellular and D2D UE use orthogonal resources. The former is preferable due to spectral efficiency improvements. Nevertheless, it can bring bigger interference issues [21].

D2D communications can be particularly advantageous at enhancing cell coverage and edge throughput, where the signals used to be weaker [20]. Out of coverage cell-edge users can relay their transmission to the BS by establishing a D2D link with devices in vicinity. Such situation can also be crucial in disaster recovery where some areas loose network coverage [20]. A D2D implementation based on using nodes as a virtual infrastructure to enhance system coverage is provided in [113]. Using a model which takes into account interference management, power control, and the round-robin scheduling technique, cell-edge capacity improvements of $200 \%$ to $300 \%$ were achieved. The authors of [114] test an approach with both full duplex amplify and forward relay nodes, and D2D communications. The objective is to assess the coverage probability in a network modeled by an homogeneous spatial Poisson point process. Comparisons were performed between cellular networks without relays, with relays, and D2D communications assisted by relays. For a SIR threshold of $10 \mathrm{~dB}$, a significant increase in the coverage probability was obtained with the results showing an improvement around $40 \%$ for a D2D relay assisted communication when compared to the standard cellular network without relays.

D2D communications can also be separated in single-hop and multi-hop. In the single-hop approach, the source and the destination communicate directly, while in a multi-hop scenario, several devices operate as intermediate nodes between the transmitter and the receiver. Due to the higher number of intermediate devices, a multi-hop configuration can improve the link quality. It influences the network coverage due to a better performance in delay and delivery for longer communication distances [115], [116], [117]. Recent research, such as [118], has been discussing the coverage definition in D2D communications. The usual SINR threshold may be limited in this scenario since the relays are not fixed. Once a new UE is associated to a relay, the available resources are dependent of the relay's backhaul. These available resources in the backhaul change dynamically, which influences the resources allocated to a specific D2D communication with impact on coverage. In [118], the authors formulate the coverage definition in order to maximize the DL rate. They use a combination of the received SINR and a dynamic resource allocation constraint. The results show an increase in the coverage area provided by a D2D relay as well as spectral efficiency improvements.

Despite these advantages, there are some issues that need to be addressed in D2D communications. These include the interference management in underlay inband category, the handover criteria, which depends on the QoS requirements of the devices, and the resources availability in the cells, as well as handover techniques for high speed users. Furthermore, lack of standardization and device battery consumption are still to be troubleshooted [21], [20]. In order to improve the coverage quality provided by a D2D system and to overcome some of the limitations listed above, the authors of [119] use several techniques to address different problems 
in this communication type. An optimization scheme, using transmission power adjustment, underlay spectrum sharing, and in-band D2D modes, avoiding frequency reuse within the 1-hop communication range for interference mitigation, is proposed. In addition, the authors consider multiple QoS metrics for comprehensive performance of relay links, and a greedy algorithm based on a distributed local search for overhead reduction.

4) Distributed Antennas System: The DAS is an antenna deployment scenario. The transmission points are distributed throughout the cell using coax cable or fiber, instead of being centrally located on a single tower [27]. The connection to the home BS uses a high bandwidth low-latency dedicated link, creating more uniform coverage inside the cell. They can be operated as relays to enhance coverage and capacity in events venues, and providing a powerful solution for indoor coverage environments. Hence, on the one hand, in some scenarios DAS can be considered as a competing technology for relays and small cells. On the other hand, it can also be a complementary technology since relays and small cells can have distributed antennas. The main difference between these two techniques is that the DAS does not use wireless spectrum between the antenna and the BS, and the antennas are fully coordinated by the central entity. This provides a wider coverage area than what is achieved with the small cell deployments [37], [27]. In [120], the authors compare the performance of a 7 microcellular and a 7 sector DAS system. Despite the micro-cellular provides more capacity, since each one of the 7 micro-cells is a dedicated BS, it increases the interference mainly for the celledge users. Furthermore, with frequency reuse-factoring, the interference tends to be even higher for the micro-cell system, while this situation is not noticeable with the DAS. Therefore, the DAS provides a powerful coverage enhancement solution, mainly for the cell-edge, since it can better mitigate the effects of interference. Moreover, when combined with technologies such as MIMO with diversity gains, the capacity limitations can be overcome [27].

Within the existing challenges, power control and coexistence with heterogeneous infrastructure must be addressed when targeting future cellular deployments. In the former, due to the fact that the UE estimates the DL path loss based on the average received power (which in this scenario is a combination of the measurements from all transmission points) the UE will not be aware of the different power classes of the transmitting nodes. Thus, the UL power control might be inaccurately measured. The implementation of DAS in cellular networks with relays, femtocells or picocells, also complicates the interference management. It results in an increased diversity of out-of-cell interference sources, creating cross-tier interference. Further research is needed to address this coexistence in a HetNet environment [27].

\section{Infrastructure Sharing}

According to Ofcom, the U.K. spectrum regulator, there are still several areas in the U.K. that are just covered by one mobile operator, or none in some more isolated places [121]. Ofcom suggests that a rural wholesale access could increase up to $2-3 \%$ the areas covered by two or more network operators using $700 \mathrm{MHz}$ carriers, and up to $10 \%$ for the operators using higher carriers [122]. This wholesale access means that costumers are allowed to roam onto one another's networks. Infrastructure sharing also allows the operators to reduce the deployment costs for achieving the coverage requirements defined by the regulator entities. The U.K. has recently defined an operation, termed as Shared Rural Network, which aims to provide more and better coverage to overcome the persistent problem of poor mobile coverage in the countryside. An additional $16,000 \mathrm{~km}$ of covered roads is also targeted [123]. This is being achieved through an agreement for operators to share the existing masts and infrastructure, closing almost all not-spot-areas. These not-spot-areas are locations served by at least one but not all operators [123].

\section{Masts}

While in the past one important constraint on network planning was the maximum masts height, allowing them to be masked by the environment elements, such as buildings and trees, nowadays it represents a network issue. Shorter masts are more prone to blockage of the signals, threatening the coverage reliability, which is a crucial factor for cellular networks. To avoid a significant loss in the entire "Fresnel Zone" of a radio transmission, it is recommended that a tree should be at least $3 \mathrm{~m}$ below the BS height. It is estimated that when the BSs and the surrounding trees or buildings have similar heights, the coverage can be reduced as much as 70\% [7]. In [124], where data from trees height in the U.K. is available, it is suggested that masts with $10 \mathrm{~m}$ high can suffer from $30 \%$ of trees blockage, and $10 \%$ for $15 \mathrm{~m}$ masts. According to U.K. government regulations, the height of a BS mast in the U.K. cannot exceed $25 \mathrm{~m}$ in non-protected areas and $20 \mathrm{~m}$ in protected areas [125]. Fulfilling these constrains and increasing the mast height to $25 \mathrm{~m}$, result in dropping the probability of blockage to less than $1 \%$. This can be considered as a significant enhancement for a short increment in BS height [7].

\section{E. Unmanned Aerial Vehicles}

The debate around UAVs is growing in the telecommunication area. The mobility and flexibility they provide together with the fact that they can either be used as aerial BSs or operate as relays, enhancing the network coverage, is resulting in increasing interest around this solution [22], [126]. The UAVs can be implemented as a static AP similar to a terrestrial $\mathrm{BS}$, or deployd in a more dynamic manner in specific scenarios (isolated areas, disastrous situations). Based on the operating altitude, the UAVs can be categorized into highaltitude platforms (HAPs) or low-altitude platforms (LAPs). While the former can work at altitudes above $17 \mathrm{~km}$ in a system called stratospheric communications [127], having in general an almost stationary operation, the latter can be moved in a flexible manner [22].

The high altitude UAVs are often deployed to provide widescale coverage in large geographic areas [23]. The stratosphere represents a potential placement for these platforms. It brings 


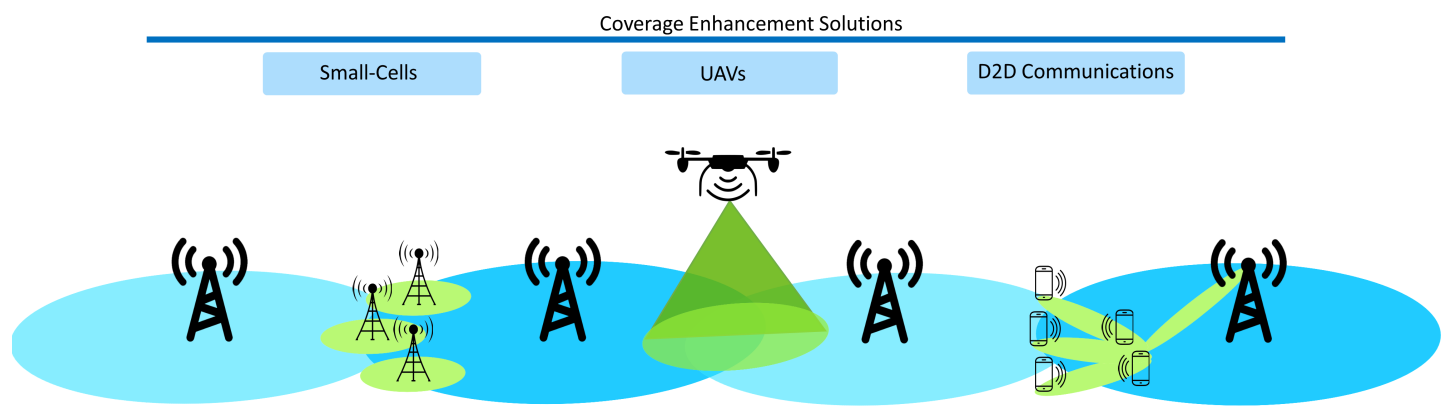

Figure 4. Examples of cell-edge coverage enhancement solutions.

together environment stability with lower wind speed and no clouds. This allows the location of quasi-stationary HAPs fed by clean solar energy, at a considerable high altitude for long range radio coverage [127]. The authors of [128] ran an experimental balloons flight test to reach optimized altitude for the deployment of zero-pressure high altitude balloons, taking into account wind and RF propagation. The tests were performed in the $145 \mathrm{MHz}$ frequency band. The results show that the longest operation duration happens in an altitude of $7 \mathrm{~km}$ with a coverage area, based on the availability of LoS component, of $718 \mathrm{~km}^{2}$. Nevertheless, despite reducing the duration in approximately 1 hour, at $20 \mathrm{~km}$ altitude a larger coverage area of $1180 \mathrm{~km}^{2}$ is achieved. Similar results were produced for coverage in [129], where a stratospheric balloon system at an altitude of $20 \mathrm{~km}$ is simulated for low dense population wide areas. Considering a coverage radius of 43 $\mathrm{km}$, with a configuration similar to LTE $(20 \mathrm{MHz}$ bandwidth and $2.6 \mathrm{GHz}$ carrier), the $5^{\text {th }}$-percentile SINR and throughput achieve values of approximately $6 \mathrm{~dB}$ and 7 Mbps respectively. These stratospheric communication systems, due to a mostly LoS operation regarding the ground UEs, also present some potential for use with higher frequencies, namely $\mathrm{mm}$-Waves. Nevertheless, problems regarding rain and atmosphere absorption, together with some unavoidable obstructions from buildings and obstacles in the ground, mainly for urban scenarios, may decrease the cell radius and raise some connectivity issues [130].

Regarding the LAPs scenario, the authors of [131] provide an analytical approach to optimize it, in order to provide maximum radio coverage on the ground. This coverage is defined as the area below the UAV where the MAPL is less than a specific threshold. It was found that the ideal altitude is achieved as a function of the MAPL. In an urban environment, the cell radius can be extended up to $7 \mathrm{~km}$ for a MAPL of $120 \mathrm{~dB}$. For this same MAPL, the cell radius becomes higher in lower density environments. Some of the main use cases for UAV regards on the operation as network coverage relays towards the ubiquitous coverage requirements [23]. When operating as relays, the UAVs are deployed to assist the existing infrastructure. For example, for rapid service recovery or base station offloading in extremely crowded areas, as in music or sports venues. In the relaying scenario, the UAVs provide wireless connectivity between two or more points, towards the exact same concept of terrestrial relays, discussed in Section IV-A.

The work in [132] assesses the deployment of a UAVs system with Non-Orthogonal Multiple Access (NOMA) operating as a relay node to extend coverage and increase the cell-edge users performance. The study compares a user near to the BS and a cell-edge user served through the UAV relay. The results show that for higher UAV transmitting power, the outage probability based on a minimum throughput threshold for the cell-edge can be lower than for users served by a terrestrial BS. Furthermore, the system throughput resulting from the cell-edge and cell-centre users combined, is enhanced around $40 \%$ and $50 \%$ comparing to an orthogonal multiple access solution. The study in [133] addresses the UAVs use for offloading of cellular hotspots, flying around the cell-edge area to serve the users with poorest channel conditions. It is initially aimed to maximize throughput by means of UAV trajectory optimization, bandwidth allocation, and user partitioning. In a second stage, an additional spectrum reuse scheme is applied. The proposed hybrid solution enhances the spacial throughput by more than 3 times when compared with a BS performance without UAV support, for a UAV transmitting power between $35 \mathrm{dBm}$ and $40 \mathrm{dBm}$. Additionally, a comparison is performed between the proposed solution and the use of micro-cell BSs at the cell-edge, targeting the cell offloading while improving the cell-edge throughput. The solution with one UAV overcomes the results with micro-cells by $34 \%$, when even 16 of them are deployed around the cell. An illustration of a UAV system operating at the cell-edge, together with the previous mentioned solutions regarding small cells and D2D communications when applied for that same purpose, is provided in Fig. 4.

Despite all of its potential, there are some challenges regarding the deployment of these UAVs solutions. New protocols must be designed, concerns with size, weight and power may limit their communication, computation and endurance capacity. Additionally, new interference management mechanisms need to be drawn, as well as the already mentioned efforts regarding trajectories and altitude optimization [22], [23].

\section{F. Satellite Communications}

Integrating satellites with cellular networks is a theme that is being discussed for a long period. In the past, satellites did not bring the efficiency of terrestrial cellular networks 
for basic services, such as voice and low data rate services, due to limitations in power and radio spectrum. Thus, when applied to mobile services, this technology was mostly used to reach specific areas of sea and air [134]. Nevertheless, that changed with technology improvement, and when heavier data services started to dominate much of the mobile traffic. Therefore, solutions encompassing both terrestrial and satellite communications started to appear, where satellites can be used for specific types of communication such as broadcasting [134]. Furthermore, satellites are a powerful solution to achieve wide area coverage, which is even more important for the future of mobile communications when the ubiquitous coverage requirements are taken into account. This solution can have an important impact in rural and remote areas, since one of its main advantages lies on the capacity dimension to enable wide geographical connectivity coverage. Therefore, it can mitigate the need for fixed infrastructures deployment such as cable links.

In addition, satellite communications are important to extend coverage connectivity for the mMTC use case [135]. However, the latency incurred by satellite links as well as the Doppler shift over the satellite channel, have remained an issue for some services, especially for geostationary (GEO) satellite systems. An hybrid architecture of terrestrial and low Earth orbit (LEO) satellites is a promising solution for future networks [136]. The LEO satellites operate at an altitude between 500 and $2000 \mathrm{~km}$ and can cover an area of 1 million $\mathrm{km}^{2}$, supporting over $50 \mathrm{Mbps}$ data rates. Moreover, due to their lower operation altitude, LEO satellites consume less energy for correct placement and allow low latency communications. In order to extend the terrestrial coverage of the 3GPP NBIoT standard, a LEO satellite constellation is studied in [137] targeting mMTC scenarios. The proposed solution is based on a unidirectional and unsynchronized link from the terminals to the satellite. A new detection algorithm is also proposed for minimization of the Doppler drift. With this solution, a reduction in the SNR of $2.5 \mathrm{~dB}$ to achieve a same value of packet error rate of $1 \%$ was obtained. Additionally, a supported path loss $20 \mathrm{~dB}$ higher than the legacy LTE was achieved.

The authors of [136] defend the service continuity advantage resulting from the dense deployment of satellites. In this scenario, the users outside the radio coverage of a terrestrial network can still have access to mobile services. This can have a considerable impact on high-speed mobility profiles, e.g., motorways, trains, airborne, and maritime platforms. The work in [138] also goes through an enabling LEO satellite solution with LTE systems, where the satellites operate as eNBs or relays. Problems related to Doppler shift, handover and attachment procedures when satellites operate as relay nodes are addressed. On the other hand, the LEO satellites mega-constellations need complex antenna tracking and double antennas at the ground stations to support seamless handover procedures between them [135]. Additionally, the interference (mainly DL aggregated interference) caused by the coexistence of $5 \mathrm{G}$ cellular networks with satellites is a big issue to be sorted [139].

\section{G. Self-Organizing Networks and Artificial Intelligence for Coverage}

The debate around the self-organizing networks (SONs) concept has recently gained new momentum. SON can be defined as an adaptive and autonomous network that is scalable, stable, and agile to maintain its desired objectives [140]. This self organization can take place in terms of network configuration, optimization, and healing. Thus the system can automatically adapt to a new BS activation, optimize a specific network parameter (such as coverage), or smoothly recover from a system failure. The SON topic is becoming even more important recently due to the expected growth and inclusion of artificial intelligence and machine learning (ML) algorithms in the telecommunication network operations. There is a vast classification of ML algorithms such as supervised, unsupervised, controllers, or reinforcement learning techniques [140]. Some work in the literature explores how these techniques can improve coverage.

The authors of [141] use neural networks, a type of unsupervised learning, for cellular cluster network coverage optimization. This technique is used for the establishment of optimized cell clustering by mitigating the interference. The presented mechanism reached a coverage improvement of $5 \%$ to $7 \%$ by optimizing the clusters size, and up to $15 \%$ when changing antenna azimuths, tilts, and beamwidth within the cells of the same cluster. The authors of [142] propose coverage improvement through the optimization of the tradeoff between KPIs. A traffic light-related control mechanism automatically triggers the reconfiguration of eNB transmission parameters to optimize a previously defined network metric. The results show that the system can adapt and mitigate the existence of coverage holes, even when a specific site is suddenly down. Similar objectives can be achieved with reinforcement learning algorithms targeting tilt optimization for coverage holes suppression in a network self-healing procedure [143].

These automation mechanisms are also massively merged with other techniques mostly designed for new cellular technologies. In [144] the authors propose an autonomous scheme to improve the network coverage in ultra-dense networks through joint power control and range expansion, which are adaptively applied to the network. The optimal coverage is measured in terms of minimal radio link failure rates. The proposed method can enhance the $5^{t h}$-percentile of the smallcell average throughput distribution significantly. In [145], the coverage of femtocells is improved by an adaptive system using information from indoor/outdoor mobility events. This not only mitigates the signalling overload regarding mobility in macro/femtocell environments, but also enhances the indoor network coverage. The proposed scheme reaches a $90 \%$ indoor coverage probability for distances up to $14 \mathrm{~m}$ by selfoptimizing the pilot power. In another study [146] involving UAVs, the system using deep reinforcement learning provides $100 \%$ coverage with a significant lower number of deployed UAVs. In some cases, for the same coverage area, the number of UAVs was reduced from 10 to 2 .

In essence, the coverage-capacity trade-off continues to be 
highlighted as a significant challenge in SON related papers. It has also been reported that the automated techniques struggle to provide very good coverage and capacity simultaneously without downgrading one of the metrics [142]. Nonetheless, network automation procedures enhance the network flexibility to adapt to scenarios where one is prioritized over the other. Apart from that, several ML algorithms need to be trained with huge amounts of data emanating from real mobile networks, which remains a big challenge. This however raises several concerns on privacy and user data sharing. Furthermore, this learning procedure must be continuously adaptive due to the inherent environment variability of telecommunication networks [147]. Additionally, the correct placement of SON functions, the distributed vs centralized deployment, conflicts among SON functions executed in different network nodes, and the solutions overcomplexity are open issues to be addressed [147], [148].

\section{H. Reconfigurable Intelligent Surfaces}

Reflecting surfaces have been highly considered across the legacy mobile communication technologies. This solution can provide or enhance coverage when the LoS component is of insufficient quality. It is a cost efficient solution compared with most of the existing alternatives discussed in this survey. The reflective surfaces are suitable for $5 \mathrm{G}$ network technologies, as they can be used to enable mm-Wave solutions by avoiding obstacles or blockages [149], [150]. On the other hand, there is a main limitation regarding these surfaces. They cannot enable the dynamic shaping of the impinging waves because the way they operate cannot be changed after implementation. This, in reality, represents one of the main drawbacks with respect to its use with high frequency carriers, which are susceptible to slight changes within the surrounding environment conditions [151], [152].

The new concept of intelligent surfaces represents an important upgrade to the passive reflector surfaces. It is anticipated to match some of the potentialities of other more common mechanisms for coverage enhancement in cellular networks [151]. The reconfigurable intelligent surfaces (RISs) consist of artificial surfaces, made of electromagnetic material which can alter the propagation of the incident radio waves by applying arbitrary phase shifts to the signals. Such solution is set to maximize the network coverage and throughput on a real-time basis [151]. The work in [151] provides a comparison which shows that the RISs can outperform a relay solution. The results under simulations for $2.6 \mathrm{GHz}$ and $28 \mathrm{GHz}$ show that for an optimized number of reconfigurable surface elements and reflection coefficient, the RISs solution provides twice the data rate for distances up to $250 \mathrm{~m}$ between source and relay/RIS. In [153], the authors assess the performance of a RIS-enhanced point-to-point multiple-input single-output system. It is shown that using an intelligent surface can extend the system coverage from $33 \mathrm{~m}$ to $50 \mathrm{~m}$, considering a target SNR of $8 \mathrm{~dB}$. These values scale with the increasing number of reflecting elements in the surface. Such result shows that the use of intelligent surfaces can avoid the need for a more costly new deployment of an additional AP or active relay.
The previous result is also supported by [154]. It is shown that for a similar multi-input single-output system and a SNR target of $15 \mathrm{~dB}$, the network coverage was extended from $110 \mathrm{~m}$ to $250 \mathrm{~m}$, with the use of a large intelligent surface. There are however, some issues regarding this solution. Due to being a recent topic, RIS requires further studies to understand how it interacts with wireless networks. This encompasses testbed experiments and validation beyond the simulation environments. The fact that these surfaces consist of nearly passive implementations raises some important challenges. The impossibility of performing channel estimation and the need for more efficient signal processing algorithms are two of these limitations [151].

\section{Integrated Solutions for Coverage}

The explored solutions for network coverage enhancement have their own flaws and limitations as previously discussed in this paper. In order to overcome their drawbacks, several studies address solutions merging two or more of those technologies. For instance, several works evaluate how interference coordination can mitigate some of the projected constraints of interference in densified networks [34], [155]. Merging UAVs and RISs together can provide promising solutions. For instance, RISs can be attached to UAVs to overcome the blockage and attenuation problems of mm-Waves [156]. Alternatively, RISs correct placement within the terrestrial network can be a strong support for received signal quality at the UAV systems [157]. SON and ML techniques can also provide important benefits for solutions involving UAVs or HetNets [146], [145]. Table IV presents a comparison of research work on solutions integrating different promising techniques. Despite the benefits resulting from these integrated solutions, new challenges also arise from them. Additionally, some of the previous identified challenges, such as the performance drop in imperfect channel state information (CSI) conditions, still remain.

To summarise, this section explored several mechanisms for coverage enhancement via innovative ways and solutions in network deployments. Although different approaches exist to address coverage issues, the implementation of each technique depends on the specific situation or scenario. For example, the HetNets can enhance coverage by increasing the density of available APs, thus reducing the propagation distance, and increasing the LoS probability. However, this is a very expensive technology in terms of deployment costs and energy consumption, which makes it an unviable solution for rural environments. Furthermore, a significant increase in network interference is expected. This can be critical for coverage if effective interference management mechanisms are not designed. Cell radius extension mechanisms through relays or D2D communications are powerful solutions to reach uncovered areas. They can extend the cell coverage area, solve performance problems at the cell-edge, and even off-load the BS. Nonetheless, such mechanisms bring new challenges such as new sources of interference. In addition, they are 
Table IV

COMPARISON OF INTEGRATED SOLUTIONS FOR NETWORK COVERAGE ENHANCEMENT.

\begin{tabular}{|c|c|c|c|c|}
\hline $\begin{array}{l}\text { Integrated } \\
\text { Techniques }\end{array}$ & Citations & Remarks & Advantages & Limitations \\
\hline $\begin{array}{l}\text { Femtocells } \\
\text { \& SON }\end{array}$ & [145] & $\begin{array}{l}\text { The femtocell performance is dynamically } \\
\text { self-optimized by analysis of mobility events } \\
\text { from indoor and outdoor users. }\end{array}$ & $\begin{array}{l}\text { Optimized indoor coverage, minimization of leakage } \\
\text { pilot signaling to outdoor, and mitigation of mobility } \\
\text { events overhead. }\end{array}$ & $\begin{array}{l}\text { Acquisition of mobility events. } \\
\text { No details on complexity or processing } \\
\text { time. }\end{array}$ \\
\hline $\begin{array}{l}\text { UAVs, } \\
\text { CF Networks, } \\
\text { \& ML }\end{array}$ & {$[146]$} & $\begin{array}{l}\text { A UAV cell-free network is provided for coverage } \\
\text { optimization in a highway entrance, supported by } \\
\text { reinforcement learning for optimized UAVs } \\
\text { trajectory. }\end{array}$ & $\begin{array}{l}100 \% \text { coverage in the environment under test, with } \\
\text { a significant decrease of deployed UAVs as a result } \\
\text { of optimized trajectories. }\end{array}$ & $\begin{array}{l}\text { Need for highway vehicles information. } \\
\text { Delayed operation after many thousands } \\
\text { of time-slots have elapsed. } \\
\text { Seamless handover among UAVs. }\end{array}$ \\
\hline UAVs \& RIS & {$[157]$} & $\begin{array}{l}\text { Use of RIS for UAVs cellular communication } \\
\text { enhancement to overcome the problem of poor } \\
\text { signal strength related to the downtilt of terrestrial } \\
\text { BSs. }\end{array}$ & $\begin{array}{l}\text { The use of RIS to direct the reflected radio waves } \\
\text { towards specific UAVs, avoiding blockages, allows } \\
\text { a better operation of UAV systems, even at } \\
\text { considerably higher altitudes/distances. This enhances } \\
\text { cellular coverage and throughput in UAV systems. }\end{array}$ & $\begin{array}{l}\text { Additional study on optimized distance } \\
\text { and altitude when placing RIS is missing. }\end{array}$ \\
\hline $\begin{array}{l}\text { UAVs, RIS, \& } \\
\text { Beamforming }\end{array}$ & {$[158]$} & $\begin{array}{l}\text { Jointly optimizing active beamforming at UAVs } \\
\text { and passive beamforming at RIS to take full } \\
\text { advantage of promising RSI-assisted UAV systems. }\end{array}$ & $\begin{array}{l}\text { System performance improvement when UAVs and } \\
\text { RSIs are combined, which impacts network coverage. } \\
\text { Received power maximization of ground UEs. }\end{array}$ & $\begin{array}{l}\text { Channel state information obtention is } \\
\text { fully assumed. }\end{array}$ \\
\hline $\begin{array}{l}\text { UAVs, RIS, } \\
\text { mm-Waves, } \\
\text { \& ML }\end{array}$ & {$[156]$} & $\begin{array}{l}\text { UAVs-carried RIS to enhance the performance } \\
\text { operation of mm-Waves. Reinforcement learning } \\
\text { is used to optimize the LoS component, i.e., the } \\
\text { UAV embedded RIS placement. }\end{array}$ & $\begin{array}{l}\text { The LoS component with mm-Waves is enhanced by } \\
90 \% \text { compared to static RISs. The DL performance is } \\
\text { maximized. Thus, coverage holes and shadow areas } \\
\text { are mitigated, as well as the overall network coverage } \\
\text { when using mm-Waves. }\end{array}$ & $\begin{array}{l}\text { Precise CSI is required between the RIS } \\
\text { and UE. There is no consideration } \\
\text { regarding the downtilt configuration } \\
\text { nature of terrestrial BSs. }\end{array}$ \\
\hline $\begin{array}{l}\text { UAVs, NOMA, } \\
\text { \& Relays }\end{array}$ & {$[132]$} & $\begin{array}{l}\text { UAVs are deployed as network relay nodes, } \\
\text { operating in the NOMA domain. Cell-edge users, } \\
\text { initially served by terrestrial BSs, are now under the } \\
\text { umbrella of a UAV relay node. }\end{array}$ & $\begin{array}{l}\text { Network coverage extension and increased cell-edge } \\
\text { performance. Better performance than orthogonal } \\
\text { multiple access. }\end{array}$ & $\begin{array}{l}\text { Need for further optimization of UAV } \\
\text { placement and power allocation } \\
\text { coefficients. }\end{array}$ \\
\hline $\begin{array}{l}\text { RIS, } \\
\text { mm-Wave, } \\
\text { \& Satellites }\end{array}$ & [159] & $\begin{array}{l}\text { Satellite embedded RISs are used to overcome THz } \\
\text { band propagation issues, enabling its use for high } \\
\text { capacity inter-LEO satellite links. }\end{array}$ & $\begin{array}{l}\text { High-capacity backhaul links for satellite systems. } \\
\text { This is important to support satellite operation as the } \\
\text { backbone network or network node. Thus, coverage } \\
\text { can be provided together with mMTC or eMBB } \\
\text { applications in mobile networks, taking advantage of } \\
\text { satellite positioning for coverage in rural areas. }\end{array}$ & $\begin{array}{l}\text { Beam misalignment between antennas } \\
\text { due to sharp beams and satellite speed, } \\
\text { which results in high received power } \\
\text { drops. Need for additional beam tracking } \\
\text { techniques. }\end{array}$ \\
\hline $\begin{array}{l}\text { Passive } \\
\text { Reflectors } \\
\text { \& mm-Waves }\end{array}$ & $\begin{array}{l}{[149]} \\
{[150]}\end{array}$ & $\begin{array}{l}\text { Use of passive reflectors with mm-Waves to avoid } \\
\text { obstacles, blockages, and improved performance on } \\
\text { NLOS regions. }\end{array}$ & $\begin{array}{l}\text { Coverage radius improvements from } 150 \mathrm{~m} \text { to } \\
500 \mathrm{~m} \text { in urban environments [144]. Considerable } \\
\text { improvements of 5th-percentile received power } \\
(10 \text { to } 20 \mathrm{dBm}) \text { [145]. }\end{array}$ & $\begin{array}{l}\text { Reflector size and shape need to be } \\
\text { planned for each environment. Lack of } \\
\text { adaptability to environmental changes. }\end{array}$ \\
\hline $\begin{array}{l}\text { CF networks, } \\
\text { mMIMO, \& } \\
\text { Power Control }\end{array}$ & $\begin{array}{l}{[160]} \\
{[161]}\end{array}$ & $\begin{array}{l}\text { User-centric distributed mMIMO solution for } \\
\text { inter-cell interference mitigation when deploying } \\
\text { a high number of cells with additional macro- } \\
\text {-diversity. Central units are used to manage the } \\
\text { coordination. Power control is used to handle the } \\
\text { near-far effect. }\end{array}$ & $\begin{array}{l}\text { Spectral efficiency at the cell boundaries is } \\
\text { massively increased due to the mitigation of } \\
\text { the cell-edge concept occurrence in the network. } \\
100 \% \text { better spectral efficiency than small-cells. }\end{array}$ & $\begin{array}{l}\text { Accurate CSI for joint transmission and } \\
\text { interference cancellation. } \\
\text { Some procedures continue to rely on } \\
\text { typical cellular architecture. }\end{array}$ \\
\hline ICIC \& D2D & [113] & $\begin{array}{l}\text { Power control is used for adaptive UL transmission } \\
\text { of the UE that can be used as a virtual node. The } \\
\text { transmit power varies depending on the path loss } \\
\text { increase with the distance to the serving BS. } \\
\text { Additionally, the UEs acting as virtual nodes are } \\
\text { used for cell coverage extension at the cell-edges. }\end{array}$ & $\begin{array}{l}\text { Capacity at the cell-edge is improved by } 200 \% \text { and } \\
300 \% \text { for DL and UL, respectively. This is highly } \\
\text { related to the establishment of direct links between } \\
\text { UEs to carry relayed traffic. }\end{array}$ & $\begin{array}{l}\text { Full CSI is assumed in the provided } \\
\text { solution. } \\
\text { UE UL transmit power limitations. }\end{array}$ \\
\hline $\begin{array}{l}\text { ICIC \& } \\
\text { Femtocells }\end{array}$ & {$[34]$} & $\begin{array}{l}\text { Review of different power control techniques in } \\
\text { heterogeneous femtocell networks. }\end{array}$ & $\begin{array}{l}\text { Mitigation of interference between femtocell and } \\
\text { macro-UEs while maintaining femtocell performance } \\
\text { in terms of coverage and capacity. }\end{array}$ & $\begin{array}{l}\text { Handling complex power control } \\
\text { techniques in such small BSs. }\end{array}$ \\
\hline $\begin{array}{l}\text { ICIC \& } \\
\text { Relays }\end{array}$ & {$[88]$} & $\begin{array}{l}\text { Fractional power control is used to set the } \mathrm{UE} \text { and } \\
\text { relay node transmit power. }\end{array}$ & $\begin{array}{l}\text { Improved cell-edge performance in urban scenarios, } \\
\text { and coverage range expansion. }\end{array}$ & $\begin{array}{l}\text { UE UL transmit power limitations. } \\
\text { The optimized number of RN in suburban } \\
\text { scenarios is missing. }\end{array}$ \\
\hline $\begin{array}{l}\text { ICIC \& } \\
\text { HetNets }\end{array}$ & {$[155]$} & $\begin{array}{l}\text { Presentation of enhanced ICIC techniques considering } \\
\text { the future massive deployment of HetNets based on } \\
\text { time-domain, frequency-domain, and power control. } \\
\text { Use case of macro, pico, and femtocell interaction. }\end{array}$ & $\begin{array}{l}\text { The use of time-domain eICIC through the almost } \\
\text { blank subframe technique, allowed to avoid outage } \\
\text { for the femtocell and picocell users in indoor and } \\
\text { cell-edge environments, respectively. }\end{array}$ & $\begin{array}{l}\text { High dependence of picocells and } \\
\text { femtocells from self-organizing features. } \\
\text { Backhaul design complexity due to the } \\
\text { coexistence of various types of cells. }\end{array}$ \\
\hline $\begin{array}{l}\text { CoMP, RIS, \& } \\
\text { Beamforming }\end{array}$ & {$[153]$} & $\begin{array}{l}\text { Deployment of a RIS for communication assistance } \\
\text { between a multi-antenna AP and multi-single- } \\
\text {-antenna UEs, by means of joint transmit beam- } \\
\text { forming optimization between the AP and the RIS. }\end{array}$ & $\begin{array}{l}\text { Coverage can be extended from } 33 \mathrm{~m} \text { to } 50 \mathrm{~m} \text { for } \\
\text { a target SINR of } 8 \mathrm{~dB} \text {. Lower costs and energy } \\
\text { consumption of RIS comparing to other technologies. }\end{array}$ & $\begin{array}{l}\text { Complexity of actively optimize the RSI } \\
\text { phase shifters. } \\
\text { CSI acquisition for the RSI elements. }\end{array}$ \\
\hline $\begin{array}{l}\text { Small-cells \& } \\
\text { mMIMO }\end{array}$ & {$[162]$} & $\begin{array}{l}\text { Small cells are deployed together with mMIMO } \\
\text { and JT CoMP. The former is used to localize the } \\
\text { interference and improve the rank of the channel } \\
\text { matrix. The latter focuses on overcoming residual } \\
\text { inter-cell interference. }\end{array}$ & $\begin{array}{l}\text { Enhancement of cell-edge spectral efficiency } \\
\text { performance, not only between at the frontier of } \\
\text { neighbouring sites, but also in the boundaries of the } \\
\text { joint processing/transmission clusters. }\end{array}$ & $\begin{array}{l}\text { Synchronization and backhaul proper } \\
\text { operations are assumed. } \\
\text { RF chain costs and signal processing } \\
\text { complexity. }\end{array}$ \\
\hline $\begin{array}{l}\text { UDNs, } \\
\text { mm-Waves, } \\
\text { beamforming, } \\
\text { \& mMIMO }\end{array}$ & {$[163]$} & $\begin{array}{l}\text { Use of hybrid analog-digital receive and transmit } \\
\text { beamforming techniques, for mitigation of inter- } \\
\text { and intra-tier interference in ultra-dense mMIMO } \\
\text { mm-Wave heterogeneous systems. }\end{array}$ & $\begin{array}{l}\text { Overcome the interference problem of ultra-dense } \\
\text { deployments, enhancing the global UE performance } \\
\text { in heterogeneous networks, namely at the cell-edges. }\end{array}$ & $\begin{array}{l}\text { A large amount of CSI is still required for } \\
\text { beamforming. Despite the joint solution } \\
\text { mitigates the interference, the drawbacks } \\
\text { behind some technologies, such as for } \\
\text { mMIMO, still exist. }\end{array}$ \\
\hline
\end{tabular}

limited by the mobile device capabilities. For rural areas, simpler solutions such as increasing the height of the masts of existing infrastructure will have a positive impact on coverage. Infrastructure sharing between mobile operators can also be used to enhance service availability. In limited coverage and/or infrastructure scenarios, UAVs and satellites can provide wide area coverage on these locations mitigating massive costs on typical equipment installation. A summary of the main mechanisms analysed in this section is provided in Table V. Their main benefits in terms of coverage enhancement as well as some implementation challenges are highlighted. The following section focuses on another identified category highly related to network coverage. It explores the impact of different frequency bands on the coverage. 
Table V

Summary of Network DePloyments For COVERAGE ENHANCEMENT.

\begin{tabular}{|c|c|c|c|c|}
\hline & $\begin{array}{c}\text { Mechanism/ } \\
\text { Implementation Type }\end{array}$ & Coverage Benefit & \multicolumn{2}{|l|}{ Limitations } \\
\hline Densification & $\begin{array}{l}\text { - Diminishing the inter-site } \\
\text { distance. [95], [18] } \\
\text { - Deployment of more BSs: } \\
\text { - Small Cells. [106], [105] } \\
\text { - Pico-Cells. [110], [111] } \\
\text { - Femto-Cells. [106], [107], [37] }\end{array}$ & $\begin{array}{l}\text { - Enhance both coverage and capacity. } \\
\text { - Coverage Holes mitigation. } \\
\text { - Cell-Edge increasing performance. } \\
\text { - The dedicated backhaul links allow to } \\
\text { offload the macro-cells traffic. }\end{array}$ & $\begin{array}{l}\text { - Increased interference for very } \\
\text { dense deployments. } \\
\text { - Infrastructure costs. } \\
\text { - Different transmitting power within } \\
\text { each tier APs may cause: } \\
\text { - Misleading in UEs connections } \\
\text { - Load imbalance. } \\
\text { - Energy consumption. } \\
\text { - Handovers based on the RSS } \\
\text { become inefficient. } \\
\text { - Low femtocells transmit power } \\
\text { take to high indoor propagation } \\
\text { losses. }\end{array}$ & $\begin{array}{l}{[95]} \\
{[96]} \\
{[49]} \\
{[19]} \\
{[89]} \\
{[90]} \\
{[97]} \\
{[112]} \\
{[108]} \\
{[109]} \\
{[106]}\end{array}$ \\
\hline \multirow[t]{2}{*}{$\begin{array}{l}\text { Cell Range } \\
\text { Expansion }\end{array}$} & $\begin{array}{l}\text { - Relays. } \\
\text { [88], [101], [104], [100] }\end{array}$ & $\begin{array}{l}\text { - Extended coverage without backhaul links. } \\
\text { - Cell-Edge increasing performance. } \\
\text { - Could be used in a cascade deployment. } \\
\text { - Coverage extension beyond the cell } \\
\text { coverage area. } \\
\text { - Suitable for rural environments. } \\
\text { - Allow transmitting power reduction of the } \\
\text { macro-cells. }\end{array}$ & $\begin{array}{l}\text { - Different transmit power between } \\
\text { the macro-cell and relay node } \\
\text { may cause: } \\
\text { - Misleading in UEs connections } \\
\text { - Load imbalance. } \\
\text { - Interference. }\end{array}$ & [37] \\
\hline & $\begin{array}{l}\text { - D2D communications. } \\
\text { [20], [113], [114] } \\
{[116],[117]}\end{array}$ & $\begin{array}{l}\text { - Macro-cells offload. } \\
\text { - Coverage extension with significant } \\
\text { cell-edge impact. }\end{array}$ & $\begin{array}{l}\text { - Interference within UEs and APs. } \\
\text { - Faster and criterion handovers. } \\
\text { - Increased signalling and } \\
\text { equipment's battery life drain. }\end{array}$ & $\begin{array}{l}{[20]} \\
{[21]}\end{array}$ \\
\hline DAS & $\begin{array}{l}\text { - Distributed transmission } \\
\text { points within a common BS. } \\
{[27],[120],[37]}\end{array}$ & $\begin{array}{l}\text { - Wider outdoor and indoor coverage. } \\
\text { - Flexible antennas deployment location. } \\
\text { - Enhanced coverage and capacity in } \\
\text { event venues. } \\
\text { - Can be merged with relays and small cells. }\end{array}$ & $\begin{array}{l}\text { - Less overall capacity than small } \\
\text { cells deployments. } \\
\text { - Power consumption and total costs. }\end{array}$ & $\begin{array}{l}{[27]} \\
{[37]}\end{array}$ \\
\hline \multirow[t]{2}{*}{ UAVs } & $\begin{array}{l}\text { - High Altitude Platforms. } \\
\text { [127], [128], [130] }\end{array}$ & $\begin{array}{l}\text { - Wide area outdoor coverage. } \\
\text { - Stratospheric communications enabler. } \\
\text { - Provide coverage for low population } \\
\text { density areas. }\end{array}$ & $\begin{array}{l}\text { - Placement stability due to natural } \\
\text { environment characteristics, i.e., rain } \\
\text { or wind. } \\
\text { - Atmospheric signal attenuation. }\end{array}$ & [130] \\
\hline & $\begin{array}{l}\text { - Low Altitude Platforms. } \\
{[22],[23],[132]} \\
{[131],[133]}\end{array}$ & $\begin{array}{l}\text { - Coverage radius extension. } \\
\text { - Provide coverage in disaster recovery } \\
\text { scenarios. } \\
\text { - Provide coverage in isolated areas. } \\
\text { - Enhanced coverage by BS offload. }\end{array}$ & $\begin{array}{l}\text { - Interference. } \\
\text { - Need for operation trajectory and } \\
\text { altitude optimization. } \\
\text { - Trade-off between device size and } \\
\text { communication capacity. }\end{array}$ & $\begin{array}{l}{[22]} \\
{[23]}\end{array}$ \\
\hline Satellites & $\begin{array}{l}\text { - Satellites integration in } \\
\text { mobile networks. } \\
{[134],[135],[136],[137],[138]}\end{array}$ & $\begin{array}{l}\text { - Join wide area outdoor coverage with } \\
\text { heavy data transmissions. } \\
\text { - Extend terrestrial network coverage. }\end{array}$ & $\begin{array}{l}\text { - Links latency. } \\
\text { - Costly solution. } \\
\text { - Handover procedure complexity. } \\
\text { - Doppler shift. }\end{array}$ & $\begin{array}{l}{[135]} \\
{[139]}\end{array}$ \\
\hline $\begin{array}{l}\text { Intelligent } \\
\text { Surfaces }\end{array}$ & $\begin{array}{l}\text { - Reconfigurable intelligent } \\
\text { surfaces. } \\
\text { [151], [153], [154] }\end{array}$ & $\begin{array}{l}\text { - Extend cell coverage radius. } \\
\text { - Ease in adapting to environment changes. } \\
\text { - Cheaper solution. } \\
\text { - Enabler for mm-Waves and Massive MIMO } \\
\text { solutions. }\end{array}$ & $\begin{array}{l}\text { - Lack of extensive studies. } \\
\text { - Channel State Information } \\
\text { performance. } \\
\text { - Need for more efficient processing } \\
\text { algorithms. }\end{array}$ & [151] \\
\hline
\end{tabular}

\section{INFLUENCE OF FREQUENCY BANDS ON COVERAGE}

The BS cell radius or coverage range is highly dependent on the used frequencies. Fig. 5 presents the relationship between the path loss and the system frequency, considering the path loss model for urban environments from [66] at a distance of $3.5 \mathrm{~km}$ to the BS. This means that solutions such as femto- or small-cells could operate ideally with higher frequencies, since the APs are closer to the end-user. On the other hand, macrocells, satellites or UAVs may operate in lower frequencies. They can provide the coverage umbrella for the network or extended cell radius to maximize the coverage area in rural environments. Fig. 6 presents the expected cell coverage radius of these different technologies, which will vary with the operating frequency in each system.

When the theme under discussion is coverage, lower fre- quencies are always preferred since they are less susceptible to environment attenuation, allowing to achieve larger coverage radius. While $\mathrm{mm}$-Waves are being highly discussed for enhanced capacity in cellular networks, low frequencies are also of great importance for use cases such as mMTC and universal seamless coverage. In this section, different solutions based on low frequencies for coverage radius maximization are discussed. Their contribution to enable cellular use cases for seamless coverage and massive connectivity is highlighted. In addition, insights on how to maximize coverage in solutions operating at higher frequencies are also provided, with focus on beamforming.

\section{A. Low Frequencies}

Low frequencies encompass a wide range of different bandwidths, grouping the radio spectrum below the ultra high 


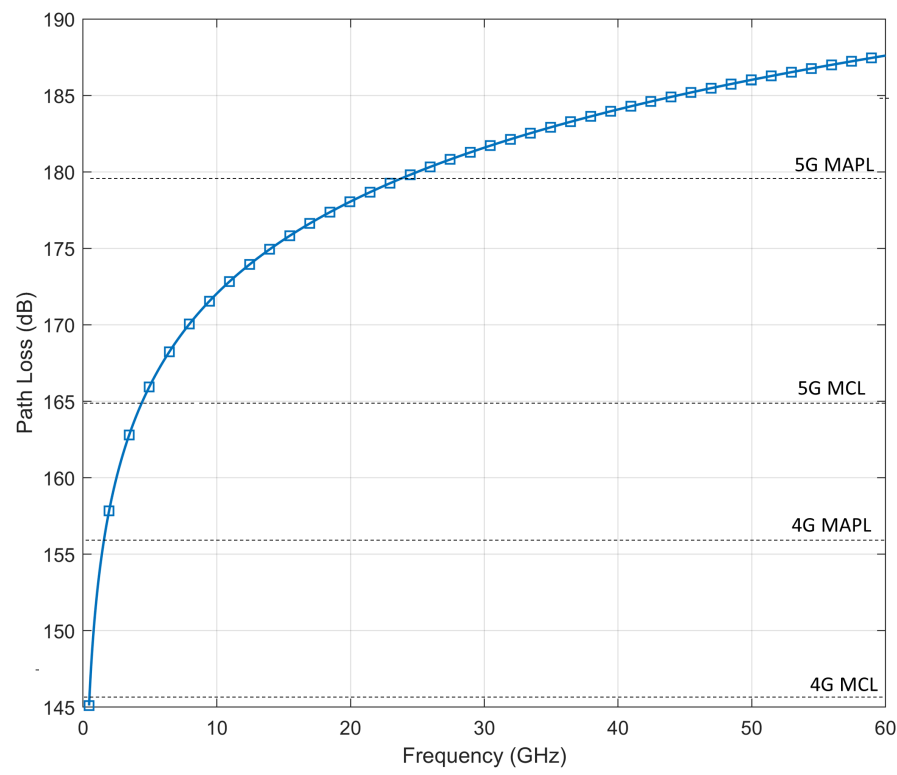

Figure 5. Influence of frequency on the radio propagation in terms of path loss, together with the MCL/MAPL LTE and 5G margins [66].

frequencys (UHFs), which is the most common in legacy cellular networks. Nevertheless, some communication types may benefit from lower frequency capabilities. For instance, the extremely low frequency (ELF) and very low frequency (VLF) bands have been studied for very long distance communications, and unusual environments such as submarine communications. These bands range between $3 \mathrm{~Hz}$ and 30 $\mathrm{kHz}$. The authors of [164] studied the use of ELF to enable submarine communications and cell ranges up to $10000 \mathrm{~km}$, for $1 \mathrm{bps}$ throughput. Similar conclusions can be drawn for the VLF scenarios in [165]. In addition, these frequency ranges are particularly useful due to their properties that allows them to be reflected in the ionosphere, and efficiently guided to global distances [166]. In this direction, it would be possible to send signals around the planet.

One of the biggest constraints regarding these applications stands in the implementation aspects. Due to their extremely large wavelengths, the deployed antennas would need to be several $\mathrm{km}$ wide. Some researchers studied the possibility of using the lower ionosphere as a large radiating antenna. This would be achievable by means of heating the ionosphere currents and change its conductivity to overcome the antenna size limitation. Based on this ionosphere heating technique, the High-Frequency Active Auroral Research Program facility was able to generate signals which were detected across the entire Alaska region [167], and at distances up to $4400 \mathrm{~km}$ [168].

Ranging between 3 and $30 \mathrm{MHz}$, providing coverage up to radius of thousands of $\mathrm{km}$, is the high frequency (HF) band. It enables applications for emergency communications in disaster areas, remote communications with aircraft and ships, NLoS military operations and distant regions lacking other communications [169]. Within their main propagation models, LoS, surface-wave and sky-wave, the latter offers the longest coverage ranges, enabling over-the-horizon or global communications. Similar to the extremely low frequency sce- narios described above, the sky-wave propagation is based on signals transmission towards the sky. These signals interact with the ionosphere and return to the Earth far beyond the horizon [169]. This idea has been discussed along history as shown in the study from [170]. The sky-wave HF communications are addressed in a ionosphere disturbance similar to what is produced by a nuclear burst. The objective relies in estimating the characteristics of the signal propagation in such conditions, and evaluate the reliability of the results provided by the statistical techniques projected for natural environments.

More recently, the more advanced technologies allowed to develop models and instruments for characterization of a wide range of ionosphere conditions. Reliable cell radius communications up to $500 \mathrm{~km}$ were achieved, envisioning emergency or natural disaster communications scenarios [171]. Another more recent application of the HF spectrum regards in its use with radars. The work in [172] studies the different phenomena which affect the operation of HF such as the daytime, range of frequencies, elevation angles, and absorption. An optimized use of the HF spectrum with the radar was reached, which allowed to track the position of an airplane up to a distance of $3000 \mathrm{~km}$.

There are other interesting technologies making use of these lower spectrum bands. Despite being gradually substituted by the Global Positioning System, Long Range Navigation (or LORAN) was the preferred system for terrestrial navigation for several years. It operates in the 90 to $110 \mathrm{kHz}$ low frequency bands (medium frequency), with coverage ranges up to $2400 \mathrm{~km}$ [173]. Located in the very high frequency range, the frequency modulation radio broadcast also represents an application benefiting from these low frequency systems. It ranges from $98 \mathrm{MHz}$ to $108 \mathrm{MHz}$ with wide area coverage capabilities.

Despite the wide coverage range properties of these frequency bands, the limitation in terms of bandwidth makes them less suitable for some of the nowadays and future needs in mobile networks. There are also the challenges regarding the antennas size and background noise. In addition, the difficulty in developing accurate models to adapt to the rapid changes in some of the suitable propagation environments such as the ionosphere, is also a key challenge for further use of these technologies [166], [169].

\section{B. Ultra High Frequencies}

In order to enable network services everywhere, the lower frequencies within the UHFs range $(300 \mathrm{MHz}$ to $3 \mathrm{GHz}$ ) are being discussed as essential in future mobile communication technologies. They can provide wide coverage and operate as an anchor or umbrella for several high capacity cellular networks deployments. The low frequency spectrum for $5 \mathrm{G}$ is set to operate in the $450 \mathrm{MHz}, 700 \mathrm{MHz}$ and $800 \mathrm{MHz}$, with standardization and re-farming being already carried out in several territories [54], [122], [174]. These bands should provide almost $100 \%$ coverage based on the SINR threshold for relatively low BS densities. These frequencies can act as potential enablers for providing coverage and fulfil the initial requirements regarding mMTC and URLLC applications. 


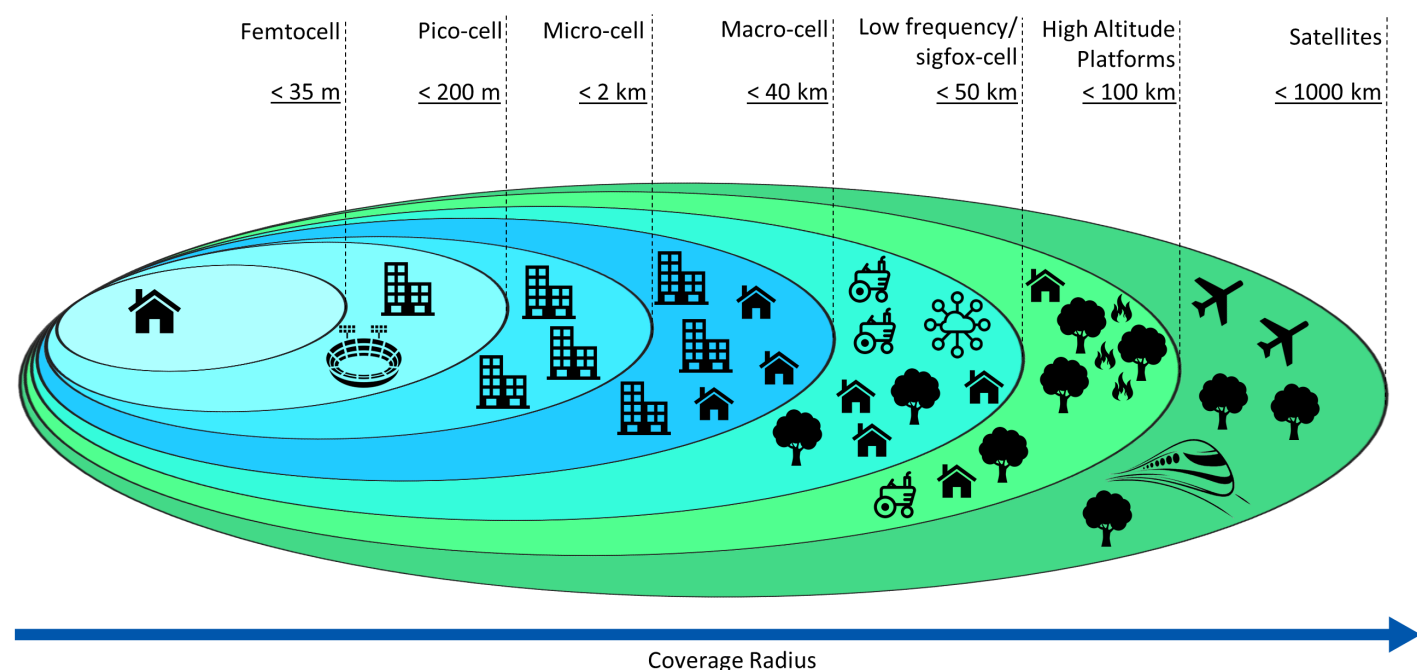

Figure 6. Projected coverage radius of different network solutions.

However, that cannot be said for the capacity. If coverage is measured in terms of data rate threshold, almost $100 \%$ coverage can be achieved in rates up to $30 \mathrm{Mbps}$ and $20 \mathrm{Mbps}$ for outdoor and indoor environments, respectively [9]. Nevertheless, using the minimum requirements of $5 \mathrm{G}$, studies show that the $64 \mathrm{Mbps}$ threshold stands at $10 \%$ of outdoor coverage for $70 \mathrm{BSs} / \mathrm{km}^{2}$, and around $7 \%$ in indoor environments [9]. Thus, solutions based on the $700 \mathrm{MHz}$ range should be ideally projected for umbrella macro cells and control-plane.

The concept of Universal Coverage is also under debate for further mobile technologies, where the rural area coverage plays an important role. In the U.K. territory, it is estimated that only $67 \%$ has LTE coverage from all operators, with almost 9\% without LTE coverage [121]. This uncovered fraction refers mainly to rural areas. Within the solutions to address this problem, spectrum management is one of the possibilities with the use of the aforementioned low frequency carriers. For this purpose, the $700 \mathrm{MHz}$ band is being migrated from digital terrestrial television to mobile services in several territories, and the 3G spectrum re-farming is being applied [54], [7]. Targeting not only the rural coverage issues but also enabling IoT systems and deep indoor coverage scenarios, several technologies are being developed using this lower layer of the UHF range. Some of them are explored below.

1) LTE-M and NB-IoT: The 5G standardization bodies aim to extend coverage into deep indoor environments, with significant penetration losses, such as parking lots and garages. This is essential for the deployment of use cases regarding mMTC and IoT, as well as for safety and emergency scenarios on these locations. Technologies such as LTE-M and NB-IoT are in the front line to provide the solution for deep coverage environments. While the last LTE-Release 8 assumes a MCL of $140 \mathrm{~dB}, 3 \mathrm{GPP}$ made some amendments to LTE Release 13 and introduced the LTE-M and NB-IoT UE categories. These technologies are projected to achieve an increased MCL of 156 $\mathrm{dB}$ and $160 \mathrm{~dB}$, respectively [16]. The former was defined for achieving $1 \mathrm{Mbps}$ throughput in $1.4 \mathrm{MHz}$ bandwidth
[91], and the latter $100 \mathrm{kbps}$ physical layer throughput in $200 \mathrm{kHz}$ bandwidth. In [91], some tests were performed in a rural area for Machine-type Communications testing, for outdoor users and indoor locations with penetration losses of $10 \mathrm{~dB}, 20 \mathrm{~dB}$ and $30 \mathrm{~dB}$. The results show that LTE$\mathrm{M}$ should be able to cover $99.9 \%$ of the light indoor users (10 dB penetration loss) while it only achieves $80 \%$ deep indoor coverage. Despite not providing total coverage as well, NB-IoT standardization allows to achieve 95\% coverage in 30 $\mathrm{dB}$ penetration loss deep indoor locations. Such technology can reach the $100 \%$ coverage by applying further signal retransmission/repitition techniques for both data and control channels. However, such process may cause the delay targets to be violated. Moreover, providing deep indoor coverage with low frequencies takes to decreased system capacity and therefore, less number of connected devices. To avoid this, micro and macro diversity, transmission time interval bundling, cell densification and power spectral density boosting may work as complementary solutions [16]. In [87], assuming a $2 \times 4$ antenna system configuration and power spectral density boosting in link level simulations, the LTE-M performance allows to achieve the 5G targets set for extremely deep indoor environments, for data rates and latency at the MCL of 164 $\mathrm{dB}$. The results show a measured data rate in UL around 363 bps and $1200 \mathrm{bps}$ in the DL, as well as $6.7 \mathrm{~ms}$ of message latency, which fulfill the requirements from Table III.

2) Long Range and Sigfox: Long Range (LoRa) and Sigfox represent an important technology for wide area coverage, mostly when the IoT use case is assumed. Due to the expected integration of multi-RATs in the following cellular networks deployments, these technologies could play an important part in coverage and long range communications. LoRa mostly operates in the 433,868 or $915 \mathrm{MHz}$ frequency bands with channel bandwidth of 125,250 or $500 \mathrm{kHz}$. The link budget of LoRa assumes a MAPL of $150 \mathrm{~dB}$, which according to this technology characteristics, would achieve a $800 \mathrm{~km}$ coverage in pure free space [175]. However, in reality this value is 
much lower. LoRa coverage highly depends on the spreading factor used, which varies between 7 and 12. Higher values of spreading factor enable longer coverage radius, although with less transmitted bits [176].

The field measurements took from [177], in Paris, allowed to assess the coverage performance of LoRa in distances ranging between $650 \mathrm{~m}$ and $3400 \mathrm{~m}$ to the LoRa gateway. The results show that for a spreading factor of 7 , approximately $85 \%$ of packets correctly reach a destination within $650 \mathrm{~m}$ of the gateway. Nevertheless there is no received packets when the distance is higher than $2800 \mathrm{~m}$. Such a situation can slightly vary depending on the existing obstacles or shadowing. On the other hand, when the spreading factor is increased to $12,100 \%$ packet delivery ratio is assured at shorter distances (up to 1400 $\mathrm{m}$ ) and at least $40 \%$ is achieved up to $3400 \mathrm{~m}$. A coverage range assessment was also conducted in Oulu, Finland [178]. Two different scenarios, ground and water, were tested. In the ground scenario, in distances up to $5 \mathrm{~km}$, a delivered package success ratio of $80 \%$ was achieved, with $60 \%$ for distances up to $10 \mathrm{~km}$. In the water this value is higher, with $70 \%$ of successful delivered packages for distances up to $15 \mathrm{~km}$. The difference between the ground and water scenarios has mainly to do with the obstructions, which are quite less in an open water environment, resulting in a lower path loss exponent. The higher coverage range in the ground tests comparing with [177] may be related with the BS or gateway placement. While in the first study the gateway was placed in the second floor of a house, in the second study, the LoRa node is placed in the top of a $24 \mathrm{~m}$ height building. Despite showing in general smooth coverage, LoRa based solutions present some susceptibility to mobility, since the packet loss when used with moving sensors can go from $20 \%$ to $45 \%$ depending on the deployment environment [176].

Despite starting to loose some importance in recent years relatively to concurrent solutions as LoRa, or more recently, the NB-IoT, Sigfox still operates in some areas. Sigfox operates on the $868 \mathrm{MHz}$ frequency band, with the spectrum divided into 400 channels of $100 \mathrm{~Hz}$. It operates in ultranarrow band at $100 \mathrm{bps}$, with an MCL of $158 \mathrm{~dB}$ and 161 $\mathrm{dB}$ for the UL and DL respectively [179], and up to 164 $\mathrm{dB}$ MAPL. The Sigfox BSs can cover a range of 20 to 50 $\mathrm{km}$ in rural areas and 3 to $10 \mathrm{~km}$ in urban environments. The study in [179] compares the coverage performance of Sigfox, LoRa and NB-IoT. A real site covering an area of $8000 \mathrm{~km}^{2}$ in Denmark is used. For users experiencing an additional penetration loss of $20 \mathrm{~dB}$, almost $100 \%$ coverage is provided by the Sigfox system which is side by side with the NB-IoT and slightly ahead of LoRa (97\%). Additionally, for deep indoor environemnts, with $30 \mathrm{~dB}$ additional penetration loss, Sigfox stands just below NB-IoT, with $85 \%$ and $90 \%$ coverage, respectively. The LoRa system just achieves a value of $76 \%$.

In terms of outdoor coverage, all the technologies under comparison achieved more than $99 \%$ coverage in the tested area, encompassing both rural and urban environments [179]. Similar conclusions were drawn in [180]. Furthermore, it was verified that when increasing the inter-site distance from 2 to 4 $\mathrm{km}$, Sigfox continues to provide good coverage, with less than
$5 \%$ outage probability for both outdoor and indor users with 20 $\mathrm{dB}$ of additional penetration loss. Despite the good results in terms of coverage, Sigfox presents higher blocking probability, duty cycle violations, and very low capacity performance, which make it less appealing than other technologies such as LoRa and NB-IoT [180], [181].

\section{Super High Frequencies}

The spectrum congestion is becoming a problem as a consequence of the deployment of several wireless communication technologies operating mostly in the UHF band, namely the four mobile legacy technologies. Therefore, super high frequencies or mm-Waves have been highly explored in recent years. They represent $90 \%$ of the allocated radio spectrum, falling in the $3 \mathrm{GHz}$ to $300 \mathrm{GHz}$ frequency range [182]. This band is mostly unlicensed, which is attractive for network providers, due to the difficulty and costs of dealing with the most common used bands. Apart from the congestion problem, mm-Waves provide very wide bandwidths to face the network capacity demands of future cellular networks.

However, there are significant technical issues regarding the deployment of cellular networks based on mm-Waves systems, mainly in terms of coverage. These frequencies are susceptible to drastic attenuation and shadowing, where the signal can suffer from increased path loss and channel intermittency, resulting in very short coverage radius [182]. Even in a LoS scenario, the weather conditions with the presence of rain or humidity, can influence the propagation of such waves. The same can be said for the simple position change of the human body relative to the mobile device [28]. Comparisons between different models of mm-Waves propagation suggest that the attenuation caused by foliage can be up to $50 \mathrm{~dB}$ and $75 \mathrm{~dB}$ for $100 \mathrm{~m}$ of foliage depth, in $10 \mathrm{GHz}$ and $38 \mathrm{GHz}$ frequency scenarios, respectively. Thus, the signal propagation is highly influenced by the environment obstacles and buildings.

One possible solution to assure better coverage with mm-Waves regards on APs densification, already explored in Section IV-A. Unlike the general networks which are interference-limited, mm-Waves networks are power-limited due to its narrow beams. However, for huge mm-Waves BSs density these networks can transit from power-limited to interference-limited, though the density threshold is much higher. The authors of [183] study the SINR and rate coverage probability in mm-Wave networks. It is shown that the SINR coverage probability always increases until a cell radius limit of approximately $50 \mathrm{~m}$. Without densification, the coverage probability based on the rate lower values (around $50 \mathrm{Mbps}$ ) used to be achieved $75 \%$ or $40 \%$ of the time depending on the system bandwidth. With densification that percentage was enhanced to almost $100 \%$. It is also concluded that with densification, the SINR coverage with mm-Waves can be comparable to conventional UHF networks.

The authors of [184] suggest the use of D2D relays to improve the mm-Waves networks coverage, routing around blockages. This solution presents significant gains in the DL coverage probability. For the same SINR thresholds, it is increased within $10 \%$ to $15 \%$ by enhancing communications in 
NLoS scenarios. The authors of [15] study the effectiveness of using mm-Waves with CoMP in BS diversity scenarios. Through an extensive mm-Wave BS diversity measurement campaign, the authors show that when a UE is served by multiple APs, the effect of blockage can be significantly reduced. The outage probability is reduced from $24.7 \%$ to $10.2 \%$ and $4.3 \%$ when served by two and three BSs, respectively. Additionally, it is concluded that with macro-diversity and a correct alignment between the directive beams of both UE and BS, the network interference can be significantly mitigated, up to a point that DL coordination through CoMP techniques might not be necessary. Even in the studied locations with more interference, only $20 \%$ performance gains were achieved with CoMP. Therefore, merging mm-Wave and macro-diversity is presented as a powerful and simpler mechanism to overcome some of the mm-Wave coverage issues in cellular networks. This is an alternative to the challenges regarding the complexity and imperfect channel state acquisition problems of CoMP.

Beamforming and beamsteering are two promising techniques for maximizing the performance of technologies using mm-Waves, namely for coverage. A more detailed analysis of this technique is provided below.

1) Antenna Beamforming and Beamsteering for Coverage: Beamforming is one of the main technologies for future cellular networks. It is often considered as an enabling technique for other important solutions such as mm-Wave, massive MIMO or NOMA [94]. Beamforming stands for a dynamic beam shaping and hence, the network performance can be optimized depending on the environment, context and user tracking. The concept is many times used interchangeably with massive MIMO. Both use multiple antennas to control the direction of a wave-front by weighting the magnitude and phase of individual antenna signals in an array of multiple antennas. Beamforming is expected to overcome some coverage issues related with $\mathrm{mm}$-Wave propagation. It increases the beam directivity towards the UE mitigating its propagation limitations. It can be classified in digital, analog or hybrid beamforming [94], [24], [29].

The authors of [185] use a novel 3-D ray tracing simulation to evaluate the $28 \mathrm{GHz} \mathrm{mm}$-Wave communication coverage. The test was performed in an urban area in Seoul, South Korea, in a $400 \mathrm{~m}$ range around the BS position. A comparison between the $28 \mathrm{GHz}$ and $900 \mathrm{MHz}$ shows an increasing 30 $\mathrm{dB}$ and $40 \mathrm{~dB}$ median path loss in the former solution, for LoS and NLoS scenarios respectively. This situation results in $50 \%$ outage probability for distances of $200 \mathrm{~m}$ to the mm-Wave AP, reaching to more than $80 \%$ if the distance is increased up to $400 \mathrm{~m}$. Nevertheless, an important range extension enhancement is shown in [30], where the authors test a mm-Wave solution with adaptive hybrid beamforming. The coverage is assessed based on BLER. Some interesting gains in terms of coverage extension were achieved with 1.7 $\mathrm{km}$ and more than $200 \mathrm{~m}$ radius in LoS and NLoS scenarios respectively. Studies on antenna array size, where a bigger number of antennas can enhance beams gain and directivity are also part of several works [186], [187]. The results show that not only because of the increasing directivity but also due to being narrow, there is less interference within the BSs beams. This allows to improve the SINR based coverage probability around $15 \%$. Additionally in [26], the authors show that when mm-Wave are used together with beamforming and beamsteering, the average cell throughput stays just below the $1 \mathrm{Gbps}$ mark. In the $5^{\text {th }}$-percentile of the rate distribution, it achieves 500 Mbps to 700 Mbps.

NOMA and beamforming can also operate together. In a multi-beam technique several beams are transmitted in different directions, and the users in the same beam can be scheduled in the power domain [94]. The authors of [188] study the use of beamforming and NOMA, by applying an angle-based technique for user pairing, in order to overcome the beam misalignment problem. For the same transmitted power, the coverage probability increases 20 to $25 \%$ comparatively to the orthogonal resources techniques, and 5 to $10 \%$ relative to the conventional NOMA. Nonetheless, there are some important challenges related to beamforming. The amount of signalling required for beam calibration in the users direction and the control signalling for users and cell detection is considerable. Such processes are complex and may have consequences in the link establishment latency [28].

2) Light Fidelity Communications for Coverage: The requirements of future cellular technologies and the challenges regarding spectrum congestion opens up space for the potential of optical wireless communications. More specifically, light fidelity ( $\mathrm{LiFi}$ ) communications which are related with visible light, ranging between the $430 \mathrm{THz}$ to $790 \mathrm{THz}$, have been suggested mainly for indoor environments communication. LiFi consists of a complete wireless system which includes bi-directional multiuser communication, involving multiple APs [189]. This technology can use light from light-emitting diodes and creates an extremely small optical cell, called attocell, which extends the outdoor coverage to bring connectivity to indoor environments. Optical radiation does not tend to interfere with other radio waves or with the operation of sensitive electronic equipment [190]. Consequently, it can be a powerful solution for environments sensitive to electromagnetic radiation, such as hospitals or airplanes. In addition, since visible light does not propagate through walls, this feature can be exploited for interference mitigation [190]. Despite providing short coverage ranges of 2.8-3.5 m diameter, the existing infrastructure for illumination indoors can be used for such technique with interference-free bandwidth reuse [191]. A hybrid $\mathrm{LiFi}$ and $\mathrm{RF}$ system would improve indoor connectivity and capacity by offering traffic off-load from congested cellular networks [192].

The existence of different types of technologies behind the white light transmission in light-emitting diodes places a challenges on the device and the choice of modulation schemes to use. Background light which simply exists due to the sunlight may result in massive data loss. This takes to the need of slower and heavier processing and hence, increased energy consumption from the sensors at the receiving device, that represents an important bottleneck for this technology [189]. This technology also needs additional backhaul connections with the external network, enabling horizontal and vertical handover procedures, managing the amount of handovers due 
to blockages and small cell size, and CSI acquisition [191].

\section{Spectrum Sharing for Network Coverage}

The discussion around the spectrum sharing concept has been rising in the last few years. It represents an efficient way of using the network frequency resources, standing as a solution to overcome the problems regarding spectrum congestion. Spectrum sharing can be applied whether multiple users are doing a similar type of use, as for business radio users, or for multiple different uses such as satellites and fixed links [193]. Furthermore, the spectrum sharing solutions can also be categorized in spectrum access type, where frequency, geography and time are the main domains. By efficiently sharing resources within different network operators, the coverage can be improved mainly in rural areas where these solutions can more easily be applied. This technology can also take an important role in the coverage issues resulting from higher frequencies use. The work in [194] shows that a $3.5 \mathrm{GHz}$ Time Division Duplex (TDD) signal can have $11 \mathrm{~dB}$ and 19 $\mathrm{dB}$ additional attenuation for DL and UL respectively when compared with a $1.8 \mathrm{GHz}$ Frequency Division Duplex (FDD) band. Therefore, due to lower transmitting power and less available slots, the UL procedure is the limiting factor here. By using spectrum sharing techniques, the TDD carrier can be paired with the UL part of a FDD band overlapped with LTE. This results in two available UL carriers. Thus, the cell-edge user can employ either the low-frequency band for coverage or the higher-frequency for capacity. This technique allows that a $3.5 \mathrm{GHz}$ solution achieves a coverage radius similar to the LTE, overcoming the coverage-capacity trade-off.

This section discussed the frequency bands typically used for mobile communication services. As a general rule; the lower the frequency, the wider the coverage. However, coverage planning must be aligned with other important metrics of mobile networks, such as capacity. Therefore, a balanced trade-off should exist when choosing a particular frequency band. ELF and VLF suit very specific long-range low data rate communications in isolated areas. For mobile communication technologies, the standard frequency bands range between $300 \mathrm{MHz}$ and $3 \mathrm{GHz}$. This range provides a balanced tradeoff between coverage and data rate, and can be further enhanced when deployed together with other technologies. For example, with spatial diversity the data rate can be increased keeping the same coverage quality. Due to the high attenuation, super high frequencies have been avoided when designing legacy telecommunication networks. Nonetheless, the demanding bandwidth requirements make these frequencies essential for future mobile communication. In order to overcome their inherent coverage limitations, beamforming techniques can help in extending the coverage range of such solutions. A summary of the main technologies which make use of the different frequency ranges explored in this section, is provided in Table VI. The following section addresses the main interference management mechanisms which can be applied to improve the network coverage. It particularly focuses on overcoming the interference problems at the cell-edge.

\section{INTERFERENCE MANAGEMENT FOR COVERAGE ENHANCEMENT}

Co-channel and inter-cell interference are some of the major network performance degradation causes since the very early releases of LTE [33]. This interference tends to be critical as the UE approaches the cell-edge where interference from neighbours is higher. Additionally, intra-site interference is also a concern in future mobile networks, aggravated by the increasing amount of deployed antennas in the same BS such as in massive MIMO solutions. Therefore, interference management is one of the most important topics for telecommunications in recent years, and it is especially important for coverage.

The $5 \mathrm{G}$ is expected to bring even more challenges in this field. Inter-cell interference exponentially grows due to the increasing number of devices and deployed APs, such as femtocells, small cells, and relays. Furthermore, the variation of the transmitting power of different BS types may bring imbalance in the coverage and traffic load. The trade-off between spectral efficiency and interference is also under consideration. Despite the interference mitigation resulting from the orthogonal resources allocation, the limitations in the spectrum availability may disallow such implementations. Carrier aggregation, cooperation among BSs, heterogeneity and dense deployments will also increase the interference issues. The same is anticipated for the wide use of novel communication models such as direct communications (D2D) [195], [196]. Moreover, this situation can be critical in some emerging applications and use cases, mainly with respect to low latency schemes. Consequently, overcoming interference is essential to ensure high and quality coverage in mobile networks. The interference management mechanisms are mostly divided into frequency reuse partitioning, CoMP, and ICIC [33].

\section{A. Impact of Frequency Reuse Partitioning on Coverage}

Frequency reuse partitioning is often considered a static ICIC method since it does not consider active coordination among BSs [197]. In this method, the spectrum is divided into two or more groups of exclusive sets. These sets are then allocated to specific regions of the cell, usually cell-centre and cell-edge UEs. The users near the cell centre adopt subbands of frequency reuse factor one, i.e., full frequency reuse. The users at the cell-edge adopt a factor reuse greater than one, i.e., partial frequency reuse [198]. Such mechanisms are able to improve the cell-edge users performance by extending the coverage area and mitigating the neighbouring cells interference. Therefore, with frequency reuse portioning, the cell-edge throughput as well as the overall network capacity are enhanced [199]. The authors of [199] proposed an enhanced fractional frequency reuse scheme to limit the intercell interference (ICI) at the cell-edge, enhancing its coverage. They merge most of the benefits from the more common soft frequency reuse and incremental frequency reuse methods. By reserving resources for the cell-edge users, using power allocation and interference awareness mechanisms, the edge throughput is improved as the distance to the cell increases. The results show an improvement from $80 \mathrm{kbps}$ to $100 \mathrm{kbps}$ 
Table VI

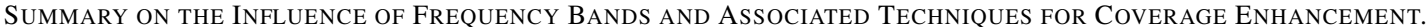

\begin{tabular}{|c|c|c|c|c|}
\hline $\begin{array}{c}\text { Coverage } \\
\text { Improvement } \\
\text { Technique }\end{array}$ & $\begin{array}{c}\text { Mechanism/ } \\
\text { Implementation Type }\end{array}$ & Coverage Benefit & \multicolumn{2}{|l|}{ Limitations } \\
\hline \multirow[t]{3}{*}{$\begin{array}{c}\text { Low } \\
\text { Frequencies }\end{array}$} & $\begin{array}{l}\text { - Spectrum Refarming for } 450 \mathrm{MHz} \text {, } \\
700 \mathrm{MHz} \text { and } 800 \mathrm{MHz} \text {. } \\
\text { [9], [54], [7], [122], [174] }\end{array}$ & $\begin{array}{l}\text { - Approximately } 100 \% \text { coverage. } \\
\text { - Achievable with low number of } \\
\text { BS deployments. } \\
\text { - Higher indoor penetration. } \\
\text { - Rural areas solution. }\end{array}$ & $\begin{array}{l}\text { - Low Capacity. } \\
\text { - Larger antennas needed. }\end{array}$ & $\begin{array}{l}{[9]} \\
{[7]}\end{array}$ \\
\hline & $\begin{array}{l}\text { - LTE-M and NB-IoT. } \\
{[16],[91],[87]}\end{array}$ & $\begin{array}{l}\text { - } 95 \% \text { deep indoor penetration } \\
\text { coverage. } \\
\text { - Increasing the MCL up to } 164 \mathrm{~dB} \text {. }\end{array}$ & $\begin{array}{l}\text { - Need for complementary techniques. } \\
\text { - Possible latency targets violation. }\end{array}$ & $\begin{array}{l}{[91]} \\
{[16]}\end{array}$ \\
\hline & $\begin{array}{l}\text { - LoRa and Sigfox. } \\
{[177],[178]} \\
{[179],[180]}\end{array}$ & $\begin{array}{l}\text { - Long range and wide coverage. } \\
\text { - MAPL of } 150 \mathrm{~dB} \text { and } 164 \mathrm{~dB} \\
\text { respectively. } \\
\text { - High deep indoor penetration } \\
\text { ( } 85 \% \text { and } 76 \% \text { respectively). }\end{array}$ & $\begin{array}{l}\text { - LoRa trade-off within spreading } \\
\text { factor and coverage range. } \\
\text { - Susceptibility to mobility. } \\
\text { - Sigfox very low capacity performance } \\
\text { and high blocking probability. }\end{array}$ & $\begin{array}{l}{[176]} \\
{[176]} \\
{[180]} \\
{[181]}\end{array}$ \\
\hline $\begin{array}{l}\text { Spectrum } \\
\text { Sharing }\end{array}$ & $\begin{array}{l}\text { - Resources sharing within } \\
\text { different operators. [193]. } \\
\text { - Mixing mmWaves with legacy } \\
\text { carriers. [194] }\end{array}$ & $\begin{array}{l}\text { - Overcome the trade-off between } \\
\text { coverage and capacity. } \\
\text { - Increased coverage radius of } \\
\text { mm-Wave frequency bands. }\end{array}$ & $\begin{array}{l}\text { - Complex resource allocation in } \\
\text { urban environments. }\end{array}$ & $\begin{array}{l}{[193]} \\
{[194]}\end{array}$ \\
\hline $\begin{array}{l}\text { Beamforming/ } \\
\text { Beamsteering }\end{array}$ & $\begin{array}{l}\text { - Shaping beams for increased } \\
\text { directivity. } \\
{[24],[29],[94]} \\
{[30],[188],[26]}\end{array}$ & $\begin{array}{l}\text { - Beam shaping towards the UEs. } \\
\text { - Improved indoor and outdoor } \\
\text { coverage. } \\
\text { - 5G technologies enabler. } \\
\text { - Increased coverage range of } \\
\text { solutions based on mmWaves, } \\
\text { Massive MIMO and NOMA. }\end{array}$ & $\begin{array}{l}\text { - Required signalling for beam } \\
\text { calibration. } \\
\text { - Latency requirements. }\end{array}$ & [28] \\
\hline
\end{tabular}

when compared to the conventional reuse-3 mechanism, which is the second best cell-edge performance.

\section{B. Impact of Inter-Cell Interference Coordination on Cov- erage}

ICIC as a concept stands for a coordination mechanism among the neighbouring cells to allocate orthogonal resources to their overlapping interfered areas. This solution encompasses procedures such as interference messages signalling, power control, and cell association optimization [195] which are explored below.

1) Overload and High Interference Indicator Mechanisms: In order to facilitate this coordination among network cells, interference related messages need to be exchanged. The X2 interface in 3GPP LTE connects neighbouring cells and it allows this information to be shared between them. Several ICIC mechanisms are developed taking into account the overload indicator $(\mathrm{OI})$ and the high interference indicator (HII) information contained inside these messages. When the eNB experiences considerable interference on some resource blocks (RBs), measured as the average interference plus thermal noise power, a message containing the OI parameter is sent to the neighbouring BSs. This indicator labels the level of interference per RB as high, medium, or low interference. The HII parameter is sent in a more proactive way, in order to prevent future high levels of ICI. In this process, the serving cell advertises to the neighbouring cells that a UE at the serving cell-edge is going to be scheduled. In this way, the neighbouring cells do their own edge UE scheduling in the specified RBs, either by lowering the power, or performing resource re-allocation [155], [200].

The authors of the work in [201] propose an OI and HII hybrid scheme for ICI mitigation. The idea behind this scheme is to use the HII messages to know which cells are contributing with more interference for a certain RB that triggered an OI message. Therefore, the serving cell can more efficiently inform neighbouring cells to carry out some scheduling adjustments. This process not only reduces the signalling overhead on X2 interface, but it also improves coverage by enhancing the cell-edge throughput performance. The results show edge-throughput improvements between $15 \%$ and $20 \%$. Nonetheless, the additional signalling that these two indicators represent could be critical in future mobile technologies. The number of neighbouring cells and hence, $\mathrm{X} 2$ interfaces, will drastically increase with densification scenarios. Additionally, there is no X2 interface between macro-cells and femtocells which makes it unuseable in HetNets deployment [155].

2) Power Control: Power control provides multiple levels of transmission power to the different cell areas identified by the frequency partitioning groups. This coordination within transmitted power and allocated frequency between BSs can be managed by a central entity or through direct communication between the BSs, in a distributed deployment. The coordination between these two procedures can be quite efficient for the cell-edge users [33]. Power control mechanisms are also being largely exploited for some of the main cellular networks technologies, namely, HetNets and D2D communications. The femtocells can be installed in an ad-hoc manner without proper planning by the operators. Consequently, the application of power control techniques become a bigger challenge due to this unsupervised and decentralized deployment [34]. However, power control techniques can be implemented in these cells through self-optimization mechanisms. For example, the femtocells can measure the interference caused by neighbouring cells and constantly adapt its transmitted power. This mechanism avoids leaks from the femtocell serving area to un- 
desirable locations and at the same time, it keeps the required coverage where the femtocell is deployed [34]. Other solutions encompass location dependent schemes which improve the SINR of the cell-edge users, reducing the outage probability. Macro-cell UE messages reporting interference can also be used by the femtocell to adapt the transmitted power based on that information [34], [202]. The latter solution is proposed in more recent studies of interference management in a two-tiers HetNets [203], where it is termed as Active Power Control. Basically, the femtocell adapts its transmitted power depending on macro-cell users feedback, through interference messages. Different steps of transmitted power can be decreased or increased depending on the content of these messages. This allows to maintain the minimum coverage requirements for the femtocell users, calculated taking into account the minimum acceptable SINR. This mechanism allows to reduce the interference on the macro user and in parallel, meet the requirements of the femtocell ones. The results show an improvement on the cell-edge macro-cell user $\left(10^{t h}\right.$-percentile worst users) approximately from $0.5 \mathrm{Mbps}$ to $2.5 \mathrm{Mbps}$.

In D2D communications, power control mechanisms can also be applied in order to minimize the covering area overlap between D2D and macro-cell UEs. This can be accomplished if the devices in the direct communication area listening to a macro-cell user. By sharing the channel gain estimation between them and the macro-cell user with the BS, the optimized transmitted power for the devices in direct communication can be calculated. This is termed as a dynamic power control mechanism [204]. Creating a separation within adjacent coverage areas of D2D and macro-cell users, the cell-edge users SINR can be increased in $3 \mathrm{~dB}$ and $7 \mathrm{~dB}$, respectively. Promising results were also achieved regarding coverage probability when using power control as enabler for D2D operation as relays and full-duplex technologies [205]. More power control schemes are continuously under investigation, towards its implementation in mobile networks. The most typical are Target SIR tracking power control (TPC), TPC with gradual removal, opportunistic power control, and dynamic SIR tracking power (DTPC) [195].

3) Cell Association: Cell association based on the RSRP/RSRQ or SINR is a major problem for future HetNets. It can create a considerable traffic load imbalance due to the different transmit power of available APs types in multi-tier networks, as already highlighted in Section IV-A. The association based on almost blank sub-frames may be a solution for the imbalance scenario. It basically consists in a technique that uses time domain orthogonalization. In this technique, specific sub-frames are left blank by the high power BS. Thus, the off-loaded users are scheduled within these blank sub-frames, avoiding inter-tier interference. A cell association mechanism based on bias-based cell range expansion, envisioning the DL coverage footprint increase, is also a contributor to overcome the load imbalance problem [195]. In this process, a positive bias is added to the BSs with lower signal strengths, increasing the area in which the UEs will connect to them. The authors of [206] propose a cell selection scheme for HetNets supported by both almost blank sub-frame and bias addition mechanisms.
With the optimized values reached for these schemes, the cell-edge spectrum efficiency was enhanced by $250 \%$ when compared to the common maximum RSRP cell association. In addition, this result is supported by an efficiency of $50 \%$ when associating UE with picocells.

The prioritized power control and resource aware cell association schemes are also under investigation for multi-tier networks, as well as joint versions of both [195]:

- Simultaneous Association to Multiple BSs.

- Cooperation and Coordination among Cells (different or same tiers).

- Channel Scheduling in Frequency Domain.

- Time domain interference coordination.

- Spatial domain techniques.

\section{Impact of Coordinated Multi-Point on Coverage}

The CoMP technology consists of coordination between the transmissions from adjacent cells. This coordination aims to reduce interference and improve cell-edge throughput, extending service coverage and enhancing cell-edge transmission. It can be divided into inter- and intra-site CoMP. The former focuses on the coordination within sectors of the same site. The latter enables the coordination among different BSs. CoMP can be classified in coordinated scheduling, coordinated beamforming and joint processing schemes [31], [207]. In [208], a CoMP solution is provided in order to increase the efficiency of the almost blank sub-frame schemes. The coordination is used with time-domain resource allocation to adjust the blank sub-frames ratio according to the traffic demand. Such a solution allowed an increase of approximately $117 \%$ in the cell-edge spectral efficiency when compared to the almost blank sub-frame approach. A coordinated scheduling scheme to assess the gains in both cell-edge throughput and spectral efficiency is proposed in [14]. The described method is based on an extended precoding matrix index. The terminals measure this index for both the serving and strongest interfering cells, together with the channel quality. Based on this information, a multicell scheduler which is coordinated among clusters of cells, makes the scheduling decisions. This multi sector scheduler allowed an improvement of $20 \%$ and $10 \%$ in the $5^{\text {th }}$-percentile throughput and spectral efficiency, respectively.

Nonetheless, CoMP schemes present some inherent implementing limitations. The channel estimation of all relevant channel components, channel prediction for time-aligned precoder design, proper cooperation areas setup and limiting feedback overhead are issues that need to be overcome. Even if cooperation mitigates inter-cell interference, a new interference type may arise from this solution, the intercooperation clusters interference. Even if cooperation mitigates inter-cell interference, a new interference type may arise from this solution, the inter-cooperation clusters interference. This results from the need of creating cooperation clusters since in several environments it is impossible for all the BSs to have information regarding the channel information and user data [32]. In addition, selecting the right group of BSs for cooperation for a specific user profile towards an optimal clustering size is another key challenge for this 
technology. Therefore, suitable cooperating AP clusters as well as mechanisms for synchronization among BSs must be found, which increases network complexity. The same can be said for efficient users selection which benefits each coordination cluster operations [31], [14].

In order to overcome some of the challenges identified above, the authors of [162] study the benefits of solutions merging joint transmission CoMP and small cells. Additionally, they provide insights on how clustering and user selection can optimize this cooperation. Cooperation leads to clustering of adjacent sites that contribute with most of the interference felt by the users in a specific area. The problem arises for the UEs at the edge of this clustering areas, since they will suffer with interference from adjacent clusters. This situation led to the need of overlapping cooperation areas, in a scheme called coverage shift. In a coverage shift scheme, the user is scheduled within different clusters. The user selection regarding each cooperation cluster is then performed with the help of small cells, with disjoint coverage within the cluster area. The first user is selected from the small cell at the cell-edge, together with other users randomly selected from other cells in the cluster. This is an iterative process, which is finished when all the users present performance gains compared to the common interference-limited transmissions. By using these sophisticated algorithms to overcome some of the cooperation limitations, $100 \%$ gains in the $5^{t h}$-percentile spectral efficiency in both small and macro-cells were achieved.

Several CoMP clustering schemes have been studied in literature. The authors of [32] define two taxonomies for CoMP clustering techniques: clustering based on self organisation and clustering based on objective function. The former is divided into static, semi-dynamic, and dynamic clustering. The overhead signalling and complexity of the system increases with the clustering mechanism to adapt to the environment changes. These changes can be linked to the user mobility, addition of new sites, sleeping cells, or load changes. Therefore, increasing the system dynamics result in enhanced performance when mitigating the clustering interference. On the other hand, this happens at the cost of significant increase in signalling and complexity of procedures such as scheduling and beamforming. In the schemes based on objective function, clustering is deployed focusing on the minimization or maximization of a specific network metric. This metric could be the spectral efficiency, backhaul bandwidth limitations, energy efficiency, or load balancing. A performance trade-off exists in both taxonomies though. The quality of the minimization or maximization of a specific objective function depends on how intelligent is the clustering system. In this direction, the author alerts for the need of creating hybrid solutions that balance the additional complexity with the CoMP gains, focusing on user-centric clustering deployed within networkcentric clusters [32]. This is quite important since reducing the complexity of CoMP techniques is a crucial factor for its deployment in cellular networks. It is important to ensure that the achieved network coverage and performance gains overcome the costs and complexity of the solution.

Additionally, some new techniques are being studied regarding centralized and adaptive DL interference coordina- tion in HetNets. These techniques are based on distributed cooperation strategies in dynamic TDD operation, enabling CoMP schemes [196]. The authors of [196] present three key interference management $5 \mathrm{G}$ enabling techniques. The aim is to prioritize the cell-edge throughput, the energy efficiency and reduce the overhead. Focusing on the cell-edge topic, two solutions are proposed. A UE-centric interference management in dynamic radio network topology, and a flexible interference management for the $5 \mathrm{G}$ air interface. The former considers a dynamic HetNet, where under the umbrella of a macro cell there are moving nodes which can enable demanddriven services and cell coverage area extension. This scenario uses adaptive interference coordination and cooperation by means of joint transmission within the access links of the moving nodes, and coordinated scheduling procedures. The flexible interference management concept relies in combining the QAM and Frequency Shift Keying (FSK) modulations, into what is termed as frequency and quadrature-amplitude modulation (FQAM). Such combination is set to change the Gaussian distribution pattern of the inter-cell interference, improving the performance of the low SINR users. The FQAM can be applied in different dimensions of the radio resources, i.e., frequency, time and space. This allows to use an agile resource management where low SINR users are scheduled from a flexible and adapted reserved resource pool, negotiated between the neighbouring cells. The mean throughput at the cell-edge spots where the moving nodes were activated increases with the higher number of deployed nodes. It is enhanced by more than $150 \%$ when 5 nodes are considered, comparing with the baseline scenario.

\section{UE Side Interference Mitigation Mechanisms for Cover- age Enhancement}

The aforementioned interference management concepts regard mainly on network-side processes. However, aiming future cellular network use cases, UE-side interference management mechanisms are being highly exploited. In reality, they are considered as enablers for the following generation of mobile communication technologies [36]. This principle can be achieved through the use of advanced receivers. The standard ones mainly presume noise-limited operational scenarios, while in modern wireless networks, most environments are interference dominated. These advanced receivers are designed to take advantage of the structure of the interfering signals. For example, an advanced receiver can decode the symbols from the interfering signal and then use the output to cancel this interferer from the received signal [36]. Comparing to standard CoMP deployments, the use of advanced receivers (UE-side) together with joint scheduling (network-side) techniques can provide average and cell-edge throughput gains of $10 \%$ and $50 \%$ respectively [36].

\section{E. Cell-Free Architecture and Coverage}

The cell-free (CF) architecture is emerging as a promising mobile network technology that aims to enhance the network performance by improving connectivity and mitigating interference. This technique avoids the conventional definition of 
cell where a specific area is designated to be served by a certain BS. Behind the main idea of CF architecture is the massive distribution of antennas across the entire network, thus operating as a distributed massive MIMO (mMIMO) system, furthermore adding a user-centric cooperation and data-sharing between the APs. Therefore, the term cell-free comes from the fact that the concept of cell does not exist from a UE perspective. It is served by a set of APs taking into account the UE rather than the cell covering area [160]. By removing the classical cell concept, this technology is expected to overcome some of the issues related to ICI.

The authors of [160] provide a detailed study on characteristics of this technology. They show that the $5^{t h}$-percentile channel gain can be enhanced by approximately $5 \mathrm{~dB}$ if a system with APs distributed every $100 \mathrm{~m}$ is deployed. In addition, if the distance between APs is reduced to $5 \mathrm{~m}$ (indoor scenario), this $5^{t h}$-percentile gain reaches $20 \mathrm{~dB}$. Furthermore, the authors of [160] tested the solution when merged together with power control techniques. For the $5^{t h}$-percentile UE spectral efficiency, using the max-min fairness power control method resulted in doubling the performance compared to the baseline approach of AP full power transmission.

The CF architecture can be deployed in a centralized manner where all the processing is carried out by a central unit. It can also be deployed in a distributed manner, where the processing is performed locally at the APs. From a coverage perspective, the work in [161] favours the centralized approach by showing a $5^{\text {th }}$-percentile spectral efficiency improvement of approximately $50 \%$ compared to the distributed approach. This results in a significant $200 \%$ additional spectral efficiency when compared to a cellular mMIMO. The authors in [209] study the performance of cell-free mMIMO systems through a SG approach. They show that the coverage probability can be significantly enhanced with a cell-free architecture when compared with small-cells. The coverage probability tends to be always higher with CF mMIMO for any number of APs or SINR threshold under testing. It was shown that a maximum improvement of approximately $20 \%$ higher coverage probability is achieved for a $100 \mathrm{APs} / \mathrm{km}^{2}$ deployment.

Despite the promising results, several challenges are associated with this technology. It requires additional user grouping and scheduling algorithms to reduce UE spatial correlation. Joint distributed AP selection schemes together with specific power allocation mechanisms for CF mMIMO also need to be designed [25]. The performance of this solution may be highly dependant on the UE location due to its low transmit power nature. In addition, the distributed $\mathrm{CF}$ architecture calls for new power control techniques. It is worth mentioning that there are still network procedures such as scheduling and information broadcast that rely on a typical cellular architecture. In addition, refined channel modelling and estimation techniques need to be developed for this new technology [160].

\section{F. Impact of Novel Rate-Splitting Interference Management on Coverage}

The rate-splitting (RS) concept has emerged as a new interference management technique. It aims to overcome limitations of conventional interference management strategies which either treat interference as noise or fully decode the interference signal [210]. It is also being discussed as a new multiple access technique with significant benefits for MIMO based technologies, dealing for example with the imperfect CSI acquisition. The RS technique is based on the transmission of common and private messages, and superimposes a common message on top of all users' private messages. This differs from other techniques which are purely based on private message transmission [211]. The minimum rate achieved with optimized precoders using RS is expected to increase between $20 \%$ and $50 \%$ depending on the number of feedback bits and the SNR quality [211].

The RS multiple access is also being studied for other promising technologies. The work in [212] studies the impact of an UAV-assisted cloud radio access network system with RS to overcome possible BS breakdown. The UAVs are used to provide coverage when a BS fails, and RS is added as an alternative interference management mechanism to treat interference as noise. This targets the maximization of the sum-rate which is increased up to $600 \mathrm{Mbps}$ in this scenario. These results are also supported by [213] with the RS technique being deployed at the central-processor of the cloud radio access network. The authors in [214] use edge caching and RS for coverage in HetNets. They show that the average-rate can be significantly maximized in coverage-aided environments when using backhaul RS. Also targeting the HetNets use case, the authors of [215] test the effect of using RS with MIMO for coverage enhancement at the cell-edge. In this process a cooperation scheme exists between cell-centre and cell-edge users. In a closed-loop spatial multiplexing scheme, one of the layers is interpreted as private while the other layer is interpreted as common. The common part of the interfering messages can be detected by the cell-edge user and cancels part of the interference. This results in improving the cell-edge spectral efficiency by upto $35 \%$.

Despite this multiple access technique based on RS presenting a promising technology, the way it can be merged with several future technologies and infrastructure, such as satellites, RIS, or sensor networks, needs further detailed studies. Additionally, this technique requires new standardization for parts of the scheme such as an identifier for the type of message (common/private), the modulation and coding scheme associated to these messages, and a separation of common message per intended UE. It is also likely to require modifications in the design of the physical and lower medium access control layers [210], [211], [216].

\section{G. Interference Management in NOMA and impact on Coverage}

NOMA is being addressed as an efficient alternative to the Orthogonal Frequency Division Multiple Access (OFDMA) technique in 5G. Apart from the time/frequency domain, NOMA also considers the power domain. Therefore, two messages can be delivered to different UEs using the same frequency but scheduled in the power domain. Thus, NOMA represents a powerful solution in terms of spectral efficiency performance [35]. However, interference continues to be a 
major issue in this scenario. It mostly happens due to intra-cell interference between the multiple users that share the timefrequency resources. Furthermore, the successive interference cancellation mechanism behind this concept [35] may increase the performance difference between the cell-centre and celledge users. This is a consequence of the cell-edge users facing worst channel conditions. Several studies in the field of interference management are being performed to overcome such issues. For example, improving coverage and minimizing the gap in performance between the cell-centre and edge users.

The authors of [217] propose an end-to-end interference management mechanism relying on NOMA and Treating Interference as Noise schemes. The Proportional Fair scheduler is used to decide which UE is going to be served. Additionally, a potential second user can be served, if the weighted sumrate with NOMA is higher than the first user rate when served in a single-user mode. Therefore, if the sum-rate with NOMA where two users are served simultaneously is higher than the single-user mode, both users are served via NOMA mode, otherwise only the first UE is scheduled. The coverage results were assessed by analysing the improvement in the $5^{t h}$-percentile of the spectral efficiency distribution. Gains of $40 \%$ for a density of $200 \mathrm{APs} / \mathrm{km}^{2}$ were verified. Alternatively, the authors of [218] propose a cooperative NOMA scheme for HetNets. The objective is to face the coverage probability problems that arise when the transmitted power is not properly allocated to the NOMA users, due to channel fading and inter-cell interference. This cooperative scheme focuses on the BSs that do not have associated users. They are coordinated to jointly transmit the signals for the far NOMA user in a particular cell. The cooperation within the void cells allowed to achieve an increasing coverage probability of $40 \%$ for the far user. However, these results depend on the number of available void BSs. Therefore, the enhancements tend to be less as the users' density rises since less void BSs exist. In their following work [219], the authors also present an enhanced version of the previous concept. In this direction, besides the BS coordination, the results regarding coverage are maximized by optimizing the power allocation scheme between the two served users.

In this section, the interference management methods with positive impact on network coverage were debated. Some of the identified methods can complement the solutions explored in the previous coverage enhancement groups, i.e., sections IV and V. Power control, for example, can overcome the power imbalance faced by D2D communications or relays and adapt the transmitted power of small-cells for interference mitigation with the macro-cell. Also targeting the power imbalance problem between different APs, the almost blank sub-frames technique can be used in the cell association process. Despite creating inter-cluster interference, CoMP is a powerful solution for interference mitigation with significant impact on the cell-edge users. It shares the same concept of beamforming since the beams are shifted and formed in a way that avoids undesired overlap between neighbouring cells. However, these techniques are complex and require high computational processing for user tracking, backhaul communication within BSs, and channel knowledge acquisition procedures. Most interference mitigation mechanisms consist of network-side procedures. However, UE-side mechanisms are also being exploited for future applications based on advanced signal processing receivers. A summary of the main mechanisms discussed in this section is provided in Table VII. In addition, Fig. 7 summarises all the explored mechanisms for coverage improvement within each of the enhancement categories defined in this article.

\section{Open IsSuES AND FUtURE RESEARCH DiRECTIONS}

The following points summarise the main identified open issues based on the findings of the paper, focusing on the main challenges with regards to coverage in future mobile network technologies. In addition, potential future research directions are underlined.

\section{A. Open Issues and Challenges of Coverage Enhancement Solutions}

This paper surveyed several technologies for network coverage enhancement. Nonetheless, significant limitations for each technology were identified. Some of them are likely to be critical and may render their implementation infeasible taking into account the network requirements for eMBB, latency, and coverage. The main identified challenges and limitations regarding the explored techniques throughout this paper are discussed below.

- The requirements of future telecommunication technologies primarily focus on capacity provision and latency reduction, though ubiquitous coverage provision is also mentioned, but not with a major emphasis. Nevertheless, this survey provides a holistic view of the increasing necessity and challenges when designing coverage enhancement solutions.

- With the stringent requirements in place for future cellular systems, we identified two main challenges to achieve the targeted objectives: the coverage-capacity trade-off and the solutions deployment costs and complexity. The former is an increasing challenge with the data volumes consumed by each UE exponentially increasing together with the bandwidth requirements of data applications. The solutions that mostly target wide area coverage are very limited in terms of bandwidth provision. This can be linked to physical limitations of signal propagation. While high frequency signals, such as the mm-Waves band, can handle much higher data volumes, they suffer from poor propagation conditions [182]. On the other hand, low frequencies can travel longer distances but transmitting smaller number of bits due to the limited available spectrum resources [7], [9]. Therefore, solutions targeting network capacity enhancement are usually poor on coverage enhancement, and they represent significant cost for massive deployment [10]. This can be considered as one of the main challenges to be handled in future cellular technologies.

- Network deployment strategies can be used to overcome the lack of coverage in mobile networks. Densification and HetNets have been utilised as powerful solutions that 
Table VII

Summary of the Interference Management Mechanisms For Coverage Enhancement.

\begin{tabular}{|c|c|c|c|c|}
\hline $\begin{array}{c}\text { Coverage } \\
\text { Improvement } \\
\text { Technique }\end{array}$ & $\begin{array}{c}\text { Mechanism/ } \\
\text { Implementation Type }\end{array}$ & Coverage Benefit & \multicolumn{2}{|l|}{ Limitations } \\
\hline $\begin{array}{l}\text { UE side } \\
\text { mechanism }\end{array}$ & $\begin{array}{l}\text { - Advanced Receivers } \\
{[36]}\end{array}$ & $\begin{array}{l}\text { - Better interference cancellation } \\
\text { at the receiver which enhances } \\
\text { UE performance at the cell-edge. }\end{array}$ & $\begin{array}{l}\text { - Processing burden for } \\
\text { the receiver. }\end{array}$ & [36] \\
\hline Static ICIC & $\begin{array}{l}\text { - Frequency Reuse Partitioning } \\
{[198],[207],[199]}\end{array}$ & \multirow{3}{*}{$\begin{array}{l}\text { - Increased overall network } \\
\text { performance, with high impact } \\
\text { on the cell-edge. } \\
\text { - Increased coverage area in } \\
\text { multi-tier networks low } \\
\text { power APs. } \\
\text { - Improved cell-edge coverage } \\
\text { by mitigating interference } \\
\text { within APs. }\end{array}$} & \multirow{3}{*}{$\begin{array}{l}\text { - Backhaul processing } \\
\text { demands. } \\
\text { - Increased latency. } \\
\text { - Channel estimation. } \\
\text { - Cooperation areas definition } \\
\text { - Inter-cooperation area } \\
\text { interference problem raised. } \\
\text { - Management of increased } \\
\text { signalling and X2 interfaces in } \\
\text { future dense deployments. }\end{array}$} & \multirow{3}{*}{$\begin{array}{l}{[14]} \\
{[31]} \\
{[155]}\end{array}$} \\
\hline CoMP & $\begin{array}{l}\text { - Joint Transmission. } \\
{[162]} \\
\text { - Cordinated Scheduling. } \\
\text { [208] } \\
\text { - Beamforming. } \\
{[196]}\end{array}$ & & & \\
\hline Active ICIC & $\begin{array}{l}\text { - Power Control. } \\
\text { [195], [33], [34], [202] } \\
\text { [203], [204], [205] } \\
\text { - Cell Association. } \\
\text { [195], [206] } \\
\text { - Overload and High } \\
\text { Interference Indicator. } \\
\text { [155], [200], [201] }\end{array}$ & & & \\
\hline $\begin{array}{l}\text { User-Centric } \\
\text { mechanism }\end{array}$ & $\begin{array}{l}\text { - Cell-free Architecture } \\
{[160],[161],[209]}\end{array}$ & $\begin{array}{l}\text { - Increased cell coverage } \\
\text { performance by mitigating the } \\
\text { existence of cell edges. }\end{array}$ & $\begin{array}{l}\text { - New mechanisms for AP } \\
\text { selection, power control and } \\
\text { power allocation are needed. } \\
\text { - Some network procedures still } \\
\text { rely on typical cellular architecture. }\end{array}$ & $\begin{array}{l}{[25]} \\
{[160]}\end{array}$ \\
\hline
\end{tabular}

\section{Coverage Related Mechanisms}

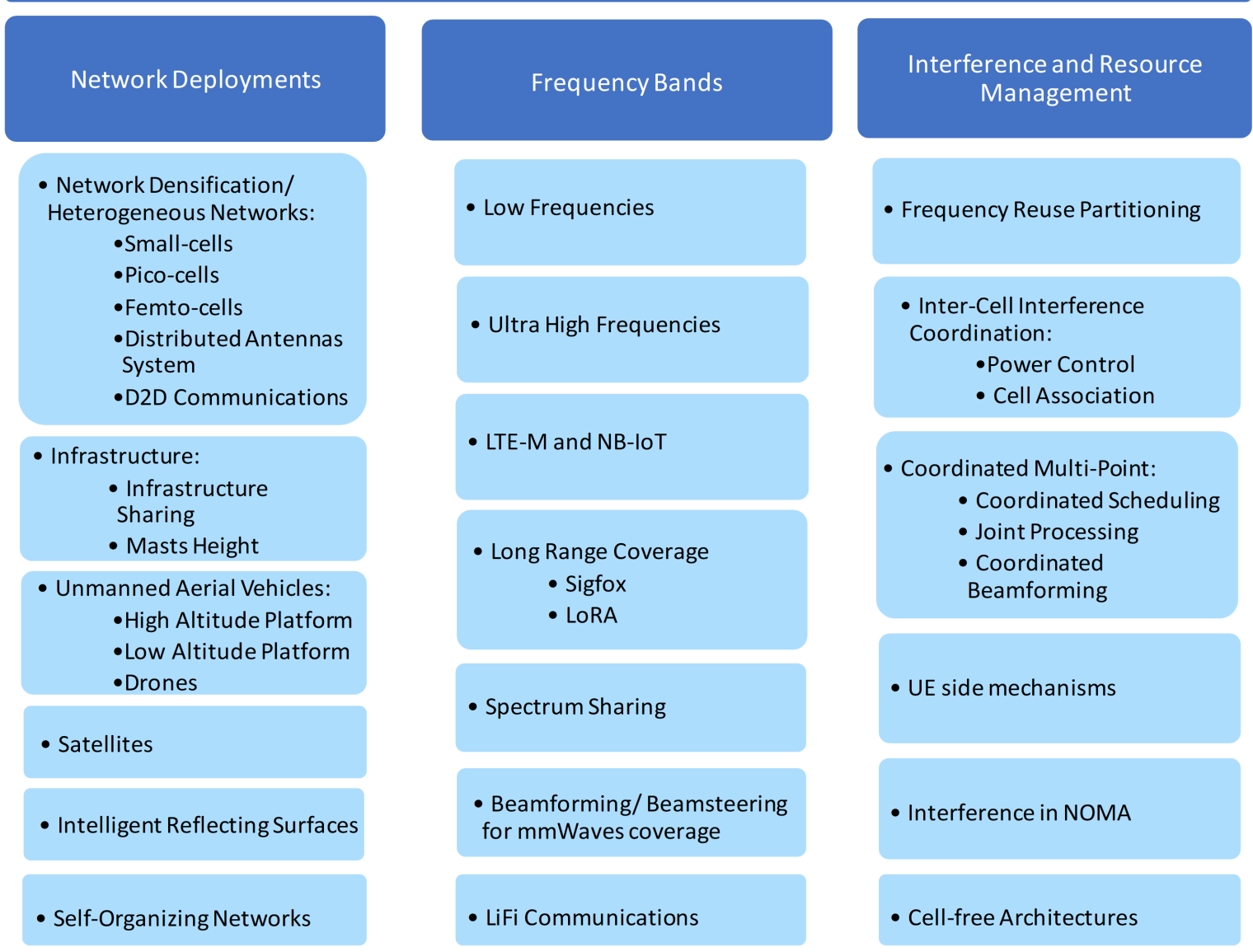

Figure 7. Coverage improvement mechanisms sorted by enhancement category. 
can address both coverage and capacity issues [94], [18]. However, these technologies bring several implementation challenges. Their deployment is expected to result in a massive increase of active APs within the network. This not only requires large investment [9], [10], but also critically augments the network interference. At some point, this can make the increase in the number of BSs redundant in terms of performance, since the gain in capacity power is counter-balanced by the increasing interference [49], [96]. Furthermore, there is still the energy consumption constraint which will grow with the massive deployment of APs. New interference management mechanisms and correct network planning are crucial challenges to enable these deployments.

- Ubiquitous and seamless coverage for the whole network are key requirements for future systems. At the moment, there are still several areas without mobile coverage. Even the more technologically advanced countries lack $100 \%$ coverage (primarily) in rural, roads, and isolated areas [121]. Enabling the 5G use cases and requirements in those areas is a difficult challenge in mobile communications. This can be traced to the high infrastructure cost and the trade-off between coverage and capacity. In this direction, guaranteeing future mobile services accessibility in rural areas places a significant challenge when deploying new cellular technologies.

- The aerial platforms encompass a powerful set of infrastructure that can be crucial for enabling high-end mobile services access everywhere. They present a flexible way of extending terrestrial network coverage, mainly when positioned at high altitudes, massively increasing the LoS communications probability. Nonetheless, mainly for HAPs, to achieve a correct placement of such infrastructure and overcoming the adversities caused by weather variations and atmosphere absorption are difficulties to be addressed [130]. Additionally, signalling and new protocols need to be designed [22]. Moreover, new interference management mechanisms are needed, mainly considering the additional and new interference types raising from the coexistence of aerial and terrestrial networks [139]. There are also additional challenges with maintenance, since this infrastructure is more difficult to physically access, and the time taken for replacement might result in increased outage time. However, moving to LEO constellation of satellites is a promising way forward but needs to overcome challenges associated with very high Doppler, frequent handovers (even between satellites), and sufficient backhauling. These solutions also share some of the cellular network dilemmas in terms of the coverage-capacity-latency trade-off. When UAVs are deployed using different frequencies, this favors the eMBB applications and mitigate the interference. Nevertheless, that might not be possible due to the scarcity of spectrum resources. On the contrary, if the UAVs are deployed within the same frequency, spectrum usage is significantly more efficient but optimized mechanisms for interference management are required. Furthermore, the latency effect due to the large propagation distance is also an important technology challenge.

- Coverage in deep indoor environments also brings new challenges for future telecommunication networks. Providing connectivity at these locations is essential for the mMTC and IoT use cases. Technologies such as NBIoT can reach deep indoor environments with up to 30 $\mathrm{dB}$ penetration loss [91]. However, these technologies alone may not fulfil the eMBB targets or be capable of handling the number of required connected devices due to their limited bandwidth and capacity. If such solutions are merged with other technologies, new challenges must be addressed. Integrated solutions can result in additional system complexity, high implementation costs, and possibly compromise the latency targets [87], [91]. In addition, DASs or femtocells which can enable indoor connectivity and provide high capacity also incur in the costs, complexity, and interference issues. More futuristic solutions such as the LiFi lack additional practical trials and need a detailed study on the background interference susceptibility, and the choice of appropriate modulation and coding scheme techniques [189].

- CoMP and beamforming are two very interesting and promising technologies to handle several issues in telecommunication networks. CoMP is considered as one of the most powerful techniques to mitigate network interference mainly at the cell-edge. Due to channel information sharing between the BS backhaul links, a shift can be applied to the transmitting beams to avoid cell overshooting. This technique suits both indoor and outdoor environments. Beamforming is often considered as a type of CoMP [31]. In addition to beam shifting, it also provides beam shaping enhancing both the capacity towards a UE and the coverage. The problem arises when the number of BSs and UE across the network is very high. The computational requirements to acquire channel state information, users tracking, and dynamically adapting the beams to the environment behaviour are very high [31], [28]. The complexity in the backhaul links to provide BS coordination is massive and it has associated latency constraints. This is not only related to the technology requirements, but also to the dynamics of communication channels [14]. Furthermore, despite the limitations in terms of coverage, it was already mentioned that mm-Wave technologies can significantly decrease network interference [15]. Therefore, within a mm-Wave system, the benefits of CoMP might not be worth the considerable complexity and deployment effort behind the technology [15]. Alternative methods should be investigated in order to create a wider range of possibilities that suit different network environments and needs.

- The cell-edge has been one of the main issues across all generations of mobile communications. The distance to the BS leads to more deterioration of signal quality due to increasing path loss. However, interference from neighbouring cells is primarily responsible for reduced network performance at these locations. The latter can be more critical in the post-5G era. As discussed in this paper, ICIC, CoMP, beamforming, DASs, or UAVs can 
optimize the QoS/QoE at these locations. These solutions can not only enhance the desired signal strength at the cell-edge by means of infrastructure deployment, but also by mitigating the interference (for CoMP, ICIC, and beamforming). Nonetheless, these techniques are complex to be applied to the entire network. Deploying an ultra-dense network together with additional techniques to handle the significant amounts of interference at the cell-edges, which grow in number if small-cells are considered, makes a telecommunication network an even more expensive and complex environment.

- The network operational costs are more than ever an area of concern. The costs around a technology deployment together with the increasing system power consumption are extremely high. This is a critical situation in an era where vendors and operators are requested to provide enhanced services and applications to customers with low-cost monthly plans [9], [10]. Therefore, on the one hand, it is essential to provide powerful and enhanced solutions for coverage and capacity to everyone at any place. On the other hand, this should be accomplished with minimized investment.

\section{B. Future Research Directions}

The following points provide some possible future research directions for coverage enhancement in telecommunication networks.

- Despite increasing the network interference, technologies such as densification and HetNets can enhance several metrics of network performance as shown in different studies [18], [106], [111]. The APs will be closer to the end-user, augmenting the LoS probability with expected major benefits for network capacity. In terms of coverage, it will bring benefit as well. More users can be served in the same serving area. In addition, small-cells can be strategically placed at the macro-cell edges to enhance the coverage in these critical areas. For coverage in indoor environments, femtocells are a potential solution. However, new solutions based on $\mathrm{LiFi}$ and $\mathrm{CF}$ networks with radio stripes [160] require further exploration with regards to the coverage-capacity trade-off, particularly in indoor scenarios. The previously stated open issues in Section VII-A regarding deployment costs are concerning. Nonetheless, mainly for high populated environments, the gains in network performance are likely to justify the investment needed for these technologies. The problems regarding the increase in network interference that can aggravate the cell-edge problem imply more studies on optimal and planned deployment of such solutions. Furthermore, this essentially alerts for the need of additional research merging densification and interference mitigation techniques [98], [99]. For example, the use of directive beams with higher frequencies might significantly decrease the interference in dense networks. While this is unrealistic for accessibility in rural and remote areas, such solutions may suit the dense urban environments [15]. Additionally, mechanisms to manage the considerable increment of handovers due to the smaller cell radius also need to be explored further.

- As aforementioned for the specific scenario of densification and HetNets, interference management mechanisms are still an active area of research. This has not only to do with the increasing number of APs, but also because the future telecommunication networks are expected to integrate different RATs, whether it is via different layers of terrestrial BSs, or through the coexistence of legacy networks with UAVs and satellites. Apart from the challenges related to signalling, placement, or latency [22], [135], the integration of these network elements brings some concerns on frequency reuse and interference management. In order to ensure a smooth coexistence of $5 \mathrm{G}$ and satellite services, interference scenarios on the component and system level must be tested to identify the performance limits. This becomes more important if both technologies are operating in the C-band, which will be part of the first $5 \mathrm{G}$ trials due to the good balance provided in terms of coverage and capacity. Beamforming is also a promising technology for interference mitigation. However, optimized solutions to operate in imperfect channel knowledge conditions are required.

- We believe that satellites, HAP or LAP systems are promising solutions to accomplish the "coverage everywhere" requirement due to their flexible and scalable deployment perspectives. In addition, the aerial platforms can be used as backbone and backhaul systems. They allow the establishment of high capacity wireless links, and can work as network nodes or routers with increased LoS connectivity. This can not only mitigate the number of deployed terrestrial BSs, but also overcome the need for the deployment of optical-fiber along considerable distances. Therefore, data transmission losses are mitigated and network deployment costs can be reduced. Nonetheless, studies focused on merging satellite and UAVs with terrestrial networks should be intensified. It is important to further investigate on the transmission channel of such solutions, together with advancements regarding the correct aerial platform placement, and environmental effects on the communication. In this direction, aerial platforms should be designed as scalable systems that suit a wide range of different scenarios providing enhanced network coverage and connectivity.

- The use of artificial intelligence in mobile networks towards the SON concept is also considered as a promising technology for future telecommunication networks. Nowadays, it is mostly applied by the use of ML techniques. ML should not be considered as a solution itself but rather a set of tools that can support several mechanisms across the entire network. This might be related to interference mitigation, network coverage, capacity, or energy efficiency. This can be achieved through the existence of automated mechanisms for BS power control, tilts or azimuth optimization, deactivation/activation of APs, and mitigation of coverage holes. In order to accomplish this, rather than just focus on the creation of specific mechanisms for network performance improvement, it 
is also necessary to take into account the deployment viability. The SON algorithms need to rapidly adapt to the network changes. In addition, they need to be opportunistic, and aligned with future cellular network requirements, in particular the latency. Therefore, we recommend further investigation on reducing the complexity of ML algorithms, the placement of such functions within the network, and the creation of a multi-party network data analytic system that allows to test these solutions under real network information. The latter would also increase the end-users and network operators trust in artificial intelligence applications for the wireless network domain, assuring the confidentiality of customers and proprietary data [147].

- We consider that sharing techniques already carried out for legacy telecommunication networks should be a common practice in future technologies. Infrastructure sharing can minimize the cost of network deployment. This also means that all operators do not need to deploy infrastructure to provide coverage in every location. Strategies can be carried out to divide the territories and assign each part to different operators, sharing the burden of infrastructure deployment across all of them. Afterwards, infrastructure can be shared between them enhancing the coverage quality and availability, mainly in rural and underpopulated areas. This should not only be targeted for terrestrial BSs, but also for future UAVs and satellite based networks. Sharing resources should also be considered for spectrum to overcome its congestion and acquisition costs. This enables sharing the time and frequency resources as well as the UL/DL decoupling. The latter relies on an additional UL carrier in a LTE FDD frequency band. This allows to use a low frequency band in the UL for coverage in parallel with the NR TDD band above $3 \mathrm{GHz}$ [194]. Such solutions overcome the coverage problem in the low power UL transmission, and benefits the balance in coverage and capacity.

- It is difficult to find a suitable solution for every network problem related to coverage. Most of the explored solutions can enhance the network coverage. Nevertheless, there is always an associated drawback. We believe that merging different concepts, in each specific scenario might be the best way to achieve the desired performance results in terms of coverage. Moreover, we also stress the need for research on simpler solutions with less deployment costs, softer impact in network interference, energy efficiency aware, and effective in achieving the proposed future technology requirements.

\section{CONCLUSIONS}

This survey provided an overview on coverage in cellular networks. It addresses methods for coverage study and improvement in mobile networks, and discusses advantages and limitations of each mechanism. It starts with the identification of several coverage definitions across the standards, academia, and forums. Afterwards, the link between the main requirements for future cellular networks and coverage was explored. Furthermore, an extensive state of the art on the main mechanisms for coverage enhancement is presented, along with insightful results and challenges for each highlighted category. This resulted in a new taxonomy for coverage optimization: the network deployments, interference and resource management, and spectrum management. This work aims to provide important insights into the existing solutions and implementation challenges for coverage. It provides a knowledge base for future work on optimal coverage solutions for each scenario and set of requirements.

\section{ACKNOWLEDGMENT}

We would like to acknowledge the support of the University of Surrey 5GIC (http://www.surrey.ac.uk/5gic) members for this work. We are also grateful to Celfinet - Outstanding Networks company for sponsoring this work.

\section{REFERENCES}

[1] Cisco, "Cisco visual networking index: Global mobile data traffic forecast update, 2017-2022," White Paper, Feb. 2019.

[2] D. G. A. Oertzen and R. Asensio, "On the technical future of the telecommunications industry," White Paper, 2017.

[3] J. G. Andrews, S. Buzzi, W. Choi, S. V. Hanly, A. Lozano, A. C. K. Soong, and J. C. Zhang, "What will 5G be?" IEEE Journal on Selected Areas in Communications, vol. 32, no. 6, pp. 1065-1082, Jun. 2014.

[4] A. Gupta and R. K. Jha, "A survey of 5G network: Architecture and emerging technologies,” IEEE Access, vol. 3, pp. 1206-1232, Jul. 2015.

[5] I. Ashraf, F. Boccardi, and L. Ho, "Sleep mode techniques for small cell deployments," IEEE Communications Magazine, vol. 49, no. 8, pp. 72-79, Aug. 2011.

[6] E. Hossain and M. Hasan, "5G cellular: key enabling technologies and research challenges," IEEE Instrumentation Measurement Magazine, vol. 18, no. 3, pp. 11-21, Jun. 2015.

[7] G. I. Centre, "5G whitepaper: Meeting the challenge of "universal" coverage, reach and reliability in the coming 5G era," White Paper, Feb. 2016, university of Surrey.

[8] K. I. Pedersen, F. Frederiksen, G. Berardinelli, and P. E. Mogensen, "The coverage-latency-capacity dilemma for TDD wide area operation and related 5G solutions," in 2016 IEEE 83rd Vehicular Technology Conference (VTC Spring), May 2016, pp. 1-5.

[9] D. Wisely, N. Wang, and R. Tafazolli, "Capacity and costs for 5G networks in dense urban areas," IET Communications, vol. 12, no. 19, pp. 2502-2510, Nov. 2018.

[10] E. J. Oughton and Z. Frias, "The cost, coverage and rollout implications of $5 \mathrm{G}$ infrastructure in Britain," Telecommunications Policy, vol. 42, no. 8, pp. $636-652,2018$, the implications of $5 \mathrm{G}$ networks: Paving the way for mobile innovation? [Online]. Available: http://www.sciencedirect.com/science/article/pii/S0308596117302781

[11] M. Xiang, L. Sun, and L. Li, "Survey on the connectivity and coverage in wireless sensor networks," in 2011 7th International Conference on Wireless Communications, Networking and Mobile Computing (WiCOM), 2011, pp. 1-4.

[12] H. ElSawy, A. Sultan-Salem, M. Alouini, and M. Z. Win, "Modeling and analysis of cellular networks using stochastic geometry: A tutorial," IEEE Communications Surveys \& Tutorials, vol. 19, no. 1, pp. 167203, First quarter 2017.

[13] A. Elnashar and M. A. El-Saidny, "Looking at LTE in practice: A performance analysis of the LTE system based on field test results," IEEE Vehicular Technology Magazine, vol. 8, no. 3, pp. 81-92, Sep. 2013.

[14] R. Irmer, H. Droste, P. Marsch, M. Grieger, G. Fettweis, S. Brueck, H. Mayer, L. Thiele, and V. Jungnickel, "Coordinated multipoint: Concepts, performance, and field trial results," IEEE Communications Magazine, vol. 49, no. 2, pp. 102-111, Feb. 2011.

[15] G. R. MacCartney and T. S. Rappaport, "Millimeter-Wave Base Station Diversity for 5G Coordinated Multipoint (CoMP) Applications," IEEE Transactions on Wireless Communications, vol. 18, no. 7, pp. 33953410, 2019. 
[16] M. Lauridsen, L. C. Gimenez, I. Rodriguez, T. B. Sorensen, and P. Mogensen, "From LTE to 5G for connected mobility," IEEE Communications Magazine, vol. 55, no. 3, pp. 156-162, Mar. 2017.

[17] M. Kamel, W. Hamouda, and A. Youssef, "Ultra-dense networks: A survey," IEEE Communications Surveys \& Tutorials, vol. 18, no. 4, pp 2522-2545, Fourth quarter 2016.

[18] D. Lopez-Perez, M. Ding, H. Claussen, and A. H. Jafari, "Towards $1 \mathrm{Gbps} / \mathrm{UE}$ in cellular systems: Understanding ultradense small cell deployments," IEEE Communications Surveys \& Tutorials, vol. 17, no. 4, p. 2078-2101, 2015. [Online]. Available: http://dx.doi.org/10.1109/COMST.2015.2439636

[19] J. Liu, M. Sheng, L. Liu, and J. Li, "Network densification in 5G: From the short-range communications perspective," IEEE Communications Magazine, vol. 55, no. 12, pp. 96-102, Dec 2017.

[20] F. Jameel, Z. Hamid, F. Jabeen, S. Zeadally, and M. A. Javed, "A survey of device-to-device communications: Research issues and challenges," IEEE Communications Surveys \& Tutorials, vol. 20, no. 3, pp. 2133 2168, Third quarter 2018

[21] A. Asadi, Q. Wang, and V. Mancuso, "A survey on device-to-device communication in cellular networks," IEEE Communications Surveys \& Tutorials, vol. 16, no. 4, pp. 1801-1819, Fourth quarter 2014.

[22] M. Mozaffari, W. Saad, M. Bennis, Y. Nam, and M. Debbah, "A tutorial on UAVs for wireless networks: Applications, challenges, and open problems," IEEE Communications Surveys \& Tutorials, vol. 21, no. 3, pp. 2334-2360, Third quarter 2019.

[23] Y. Zeng, R. Zhang, and T. J. Lim, "Wireless communications with unmanned aerial vehicles: opportunities and challenges," IEEE Communications Magazine, vol. 54, no. 5, pp. 36-42, May 2016.

[24] Y. Niu, Y. Li, D. Jin, L. Su, and A. Vasilakos, "A survey of millimeter wave (mmWave) communications for 5G: Opportunities and challenges," Wireless Networks, vol. 21, 022015.

[25] A. N. Uwaechia and N. M. Mahyuddin, "A comprehensive survey on millimeter wave communications for fifth-generation wireless networks: Feasibility and challenges," IEEE Access, vol. 8, pp. $62367-$ 62414,2020

[26] T. Bai, A. Alkhateeb, and R. W. Heath, "Coverage and capacity of millimeter-wave cellular networks," IEEE Communications Magazine, vol. 52, no. 9, pp. 70-77, Sep. 2014.

[27] R. Heath, S. Peters, Y. Wang, and J. Zhang, "A current perspective on distributed antenna systems for the downlink of cellular systems," IEEE Communications Magazine, vol. 51, no. 4, pp. 161-167, Apr. 2013.

[28] M. Giordani, M. Polese, A. Roy, D. Castor, and M. Zorzi, "A tutoria on beam management for 3GPP NR at mmWave frequencies," IEEE Communications Surveys \& Tutorials, vol. 21, no. 1, pp. 173-196, First quarter 2019.

[29] I. Ahmed, H. Khammari, A. Shahid, A. Musa, K. S. Kim, E. De Poorter, and I. Moerman, "A survey on hybrid beamforming techniques in 5G: Architecture and system model perspectives," IEEE Communications Surveys \& Tutorials, vol. 20, no. 4, pp. 3060-3097, Fourth quarter 2018

[30] W. Roh, J. Seol, J. Park, B. Lee, J. Lee, Y. Kim, J. Cho, K. Cheun, and F. Aryanfar, "Millimeter-wave beamforming as an enabling technology for 5G cellular communications: theoretical feasibility and prototype results," IEEE Communications Magazine, vol. 52, no. 2, pp. 106-113, Feb. 2014.

[31] F. Qamar, K. Dimyati, M. Hindia, K. Noordin, and A. Al-samman, "A comprehensive review on coordinated multi-point operation for LTEA," Computer Networks, vol. 123, 052017.

[32] S. Bassoy, H. Farooq, M. A. Imran, and A. Imran, "Coordinated multipoint clustering schemes: A survey," IEEE Communications Surveys \& Tutorials, vol. 19, no. 2, pp. 743-764, 2017.

[33] E. Pateromichelakis, M. Shariat, A. u. Quddus, and R. Tafazolli, "On the evolution of multi-cell scheduling in 3GPP LTE / LTE-A," IEEE Communications Surveys \& Tutorials, vol. 15 , no. 2, pp. 701-717, Second 2013.

[34] S. Saad, M. Ismail, and R. Nordin, "A survey on power control techniques in femtocell networks," Journal of Communications, vol. 8 , pp. 845-854, 122013.

[35] Z. Ding, Y. Liu, J. Choi, Q. Sun, M. Elkashlan, C. I, and H. V. Poor, "Application of non-orthogonal multiple access in LTE and 5G networks," IEEE Communications Magazine, vol. 55, no. 2, pp. 185191, Feb. 2017.

[36] W. Nam, D. Bai, J. Lee, and I. Kang, "Advanced interference management for $5 \mathrm{G}$ cellular networks," IEEE Communications Magazine, vol. 52, no. 5, pp. 52-60, May 2014.
[37] Y. A. Sambo, M. Z. Shakir, K. A. Qaraqe, E. Serpedin, and M. A. Imran, "Expanding cellular coverage via cell-edge deployment in heterogeneous networks: spectral efficiency and backhaul power consumption perspectives," IEEE Communications Magazine, vol. 52, no. 6, pp. 140-149, Jun. 2014

[38] O. O. Erunkulu, A. M. Zungeru, C. K. Lebekwe, and J. M. Chuma, "Cellular communications coverage prediction techniques: A survey and comparison," IEEE Access, vol. 8, pp. 113 052-113077, 2020.

[39] C. Phillips, D. Sicker, and D. Grunwald, "A survey of wireless path loss prediction and coverage mapping methods," IEEE Communications Surveys \& Tutorials, vol. 15, no. 1, pp. 255-270, 2013.

[40] P. Bernardin, M. F. Yee, and T. Ellis, "Cell radius inaccuracy: a new measure of coverage reliability," IEEE Transactions on Vehicular Technology, vol. 47, no. 4, pp. 1215-1226, Nov. 1998.

[41] 3GPP, "Technical Specification Group Radio Access Network; Evolved Universal Terrestrial Radio Access (E-UTRA); LTE coverage enhancements (Release 11) ," 3rd Generation Partnership Project (3GPP), Technical Report (TR) 36.824, 06 2012, version 11.0.0. [Online]. Available: https://portal.3gpp.org/desktopmodules/ Specifications/SpecificationDetails.aspx ?specificationId $=2503$

[42] 3GPP, "Technical Specification Group Radio Access Network; Study on provision of low-cost Machyne-Type Communications (MTC) User Equipments (UEs) (Release 12) ," 3rd Generation Partnership Project (3GPP), Technical Report (TR) 36.888, 06 2013, version 12.1.0. [Online]. Available: https://portal.3gpp.org/desktopmodules/ Specifications/SpecificationDetails.aspx ?specificationId $=2578$

[43] Techplayon. (2018) Maximum Coupling Loss (MCL) and Maximum Path Loss (MPL). Accessed 2019-11-29. [Online]. Available: http://www.techplayon.com/maximum-coupling-loss-mcland-maximum-path-loss-mpl/

[44] 3GPP, "Further advancements for E-UTRA physical layer aspects (Release 9)," 3rd Generation Partnership Project (3GPP), Technical Report (TR) 36.814, 03 2017, version 9.2.0. [Online]. Available: https://portal.3gpp.org/desktopmodules/ Specifications/SpecificationDetails.aspx?specificationId $=2493$

[45] G. MONARCH, "Documentation of requirements and KPIs and definition of suitable evaluation criteria," Deliverable 6.1, Sep. 2017, version 1.0

[46] S. Baroudi and Y. R. Shayan, "Evaluation of outage probability for uniformly distributed users based on signal-to-interference-plus-noise ratio," IET Communications, vol. 13, no. 9, pp. 1236-1241, Apr. 2019.

[47] D. O. Reudink, Microwave Mobile Communications, 2nd ed., W. C. James, Ed. Wiley-IEEE Press, 1993, chapter 2.

[48] R. Pattuelli and V. Zingarelli, "Precision of the estimation of area coverage by planning tools in cellular systems," IEEE Personal Communications, vol. 7, no. 3, pp. 50-53, Jun. 2000.

[49] J. G. Andrews, F. Baccelli, and R. K. Ganti, "A tractable approach to coverage and rate in cellular networks," IEEE Transactions on Communications, vol. 59, no. 11, pp. 3122-3134, Nov. 2011.

[50] S. S. Kalamkar and M. Haenggi, "Simple approximations of the SIR meta distribution in general cellular networks," IEEE Transactions on Communications, vol. 67, no. 6, pp. 4393-4406, Jun. 2019.

[51] M. Haenggi, "The meta distribution of the SIR in Poisson bipolar and cellular networks," IEEE Transactions on Wireless Communications, vol. 15, no. 4, pp. 2577-2589, Apr. 2016

[52] M. Di Renzo and A. Zappone and T. T. Lam and M. Debbah, "Stochastic geometry modeling of cellular networks: A new definition of coverage and its application to energy efficiency optimization," in 2018 26th European Signal Processing Conference (EUSIPCO), Sep. 2018, pp. 1507-1511.

[53] _ "System-level modeling and optimization of the energy efficiency in cellular networks: A stochastic geometry framework," IEEE Transactions on Wireless Communications, vol. 17, no. 4, pp. 2539-2556, Apr. 2018.

[54] "Consultation: Improving Mobile Coverage - Proposals for Coverage Obligations in the Award of the $700 \mathrm{MHz}$ Spectrum Band," Ofcom, Tech. Rep., Mar. 2018.

[55] 3GPP, "Telecommunication management; Self-Organizing Networks (SON) Policy Network Resource Model (NRM) Integration Reference Point (IRP); Information Service (IS) (Release 11) " 3rd Generation Partnership Project (3GPP), Technical Specification (TS) 32.522, 09 2013, version 11.7.0. [Online]. Available: https://portal.3gpp.org/desktopmodules/Specifications/ SpecificationDetails. asp $x$ ?specificationId $=2042$

[56] X. You, D. Wang, P. Zhu, and B. Sheng, "Cell edge performance of cellular mobile systems," IEEE Journal on Selected Areas in Communications, vol. 29, no. 6, pp. 1139-1150, Jun. 2011. 
[57] S. Kumar, I. Z. Kovacs, G. Monghal, K. I. Pedersen, and P. E. Mogensen, "Performance evaluation of 6-sector-site deployment for downlink utran long term evolution," in 2008 IEEE 68th Vehicular Technology Conference (VTC), Sep. 2008, pp. 1-5.

[58] P. Zetterberg, "Performance of three, six, nine and twelve sector sites in CDMA - based on measurements," in Eighth IEEE International Symposium on Spread Spectrum Techniques and Applications - Programme and Book of Abstracts (IEEE Cat. No.04TH8738), Aug. 2004, pp. 394-399.

[59] 3GPP, "Telecommunications Management; Subscriber and equipment trace; Trace concepts and requirements (Release 15) ," 3rd Generation Partnership Project (3GPP), Technical Specification (TS) 32.421, 06 2019, version 15.1.0. [Online]. Available: https://portal.3gpp.org/desktopmodules/Specifications/ SpecificationDetails.aspx? specificationId=2008

[60] M. Sousa, A. Martins, and P. Vieira, "Self-diagnosing low coverage and high interference in 3G/4G radio access networks based on automatic RF measurement extraction," 01 2016, pp. 31-39.

[61] A.Andrades and R. Barco and I. Serrano, "A method of assessment of LTE coverage holes," EURASIP Journal on Wireless Communications and Networking, vol. 2016, p. 236, 122016.

[62] 3GPP, "Radio Measurements Collection For Minimization of Drive Tests (MDT); Overall Description; Stage 2 (Release 15) " 3rd Generation Partnership Project (3GPP), Technical Specification (TS) 37.320, 06 2018, version 15.0.0. [Online]. Available: https://portal.3gpp.org/desktopmodules/Specifications/ SpecificationDetails.aspx? specificationId $=2602$

[63] L. Zhang, X. Gu, Z. Liu, L. Zhang, and H. Moon, "Modeling and analysis of indoor coverage probability for future $3 \mathrm{~d}$ dense mobile networks," in 2017 20th International Symposium on Wireless Personal Multimedia Communications (WPMC), 2017, pp. 247-252.

[64] J. Kim, J. Shin, S. Oh, A. Park, and M. Chung, "System Coverage and Capacity Analysis on Millimeter-Wave Band for 5G Mobile Communication Systems with Massive Antenna Structure," International Journal of Antennas and Propagation, vol. 2014, pp. 1-11, 072014.

[65] 3GPP, "Technical Specification Group Radio Access Network; Study on 3D channel model for LTE (Release 12)," 3rd Generation Partnership Project (3GPP), Technical Report (TR) 36.873, 12 2017, version 12.7.0. [Online]. Available: https://portal.3gpp.org/desktopmodules/ Specifications/SpecificationDetails.aspx?specificationId $=2574$

[66] — " "Technical Specification Group Radio Access Network; Study on channel model for frequencies from 0.5 to 100 $\mathrm{GHz}$ (Release 16) ," 3rd Generation Partnership Project (3GPP), Technical Report (TR) 38.901, 12 2019, version 16.1.0. [Online]. Available: https://portal.3gpp.org/desktopmodules/ Specifications/SpecificationDetails. aspx ?specificationId $=3173$

[67] P. Bowes. Mapinfo. Accessed 2019-12-11. [Online]. Available: https://www.pitneybowes.com/us/location-intelligence/ geographic-information-systems/mapinfo-pro.html

[68] Pitney Bowes. Mapinfo. Accessed 2019-12-11. [Online] Available: https://www.pitneybowes.com/us/location-intelligence/ geographic-information-systems/mapinfo-pro.html

[69] Forsk. Atoll. Accessed 2019-12-11. [Online]. Available: https: //www.forsk.com/atoll-overview

[70] Infovista. Planet. Accessed 2019-12-11. [Online]. Available: https: //www.infovista.com/planet/rf-planning-software

[71] R. Wireless. Ranplan. Accessed 2019-12-11. [Online]. Available: https://ranplanwireless.com

[72] Infovista. TEMS. Accessed 2019-12-11. [Online]. Available: https: //www.infovista.com/tems

[73] 3GPP, "Release 15 Description; Summary of Rel-15 Work Items (Release 15) " 3rd Generation Partnership Project (3GPP), Technical Report (TR) 21.915, 03 2019, version 1.1.0. [Online]. Available: https://portal.3gpp.org/desktopmodules/ Specifications/SpecificationDetails.aspx ?specificationId=3389

[74] ITU, "Minimum requirements related to technical performance for IMT-2020 readio interface(s)," International Telecommunication Union, Standard, Nov. 2017.

[75] 3GPP, "Technical Specification Group Radio Access Network; Requirements for Evolved UTRA (E-UTRA) and Evolved UTRAN (E-UTRAN) (Release 9) ," 3rd Generation Partnership Project (3GPP), Technical Specification (TS) 25.319, 12 2009, version 9.0.0. [Online]. Available: https://portal.3gpp.org/desktopmodules/ Specifications/SpecificationDetails.aspx?specificationId $=1174$

[76] 3GPP, "Requirements for further advancements for Evolved Unviersal Terrestrial Radio Access (E-UTRA) (LTE-Advanced) (Release 15) ", 3rd Generation Partnership Project (3GPP),
Technical Report (TR) 36.913, 06 2018, version 15.0.0. [Online]. Available: https://portal.3gpp.org/desktopmodules/Specifications/ SpecificationDetails. asp $x$ ?specificationId $=2585$

[77] 3GPP, "Technical Specifications and Technical Reports for a UTRANbased 3GPP system (Release 6) ," 3rd Generation Partnership Project (3GPP), Technical Specification (TS) 21.101, 12 2009, version 6.10.0. [Online]. Available: https://portal.3gpp.org/desktopmodules/ Specifications/SpecificationDetails. aspx ?specificationId $=544$

[78] 3GPP, "Enhanced Uplink; Overall description; Stage 2 (Release 7) "” 3rd Generation Partnership Project (3GPP), Technical Specification (TS) 25.319, 12 2009, version 7.8.0. [Online]. Available: https://portal.3gpp.org/desktopmodules/ Specifications/SpecificationDetails.aspx? specificationId=1174

[79] ITU, "Guidelines for Evaluation of Radio Interface Technologies for IMT-2020," International Telecommunication Union, Standard, Oct. 2017.

[80] 3GPP, "Study on Scenarios and Requirements for Next Generation Access Technologies; (Release 15)," 3rd Generation Partnership Project (3GPP), Technical Report (TR) 38.913, 06 2018. [Online]. Available: https://portal.3gpp.org/desktopmodules/ Specifications/SpecificationDetails.aspx ?specificationId $=2996$

[81] 3GPP, "Service Requirements for the 5G System; Stage 1 (Release 16)," 3rd Generation Partnership Project (3GPP), Technical Specification (TS) 22.261, 06 2019, version 16.8.0. [Online]. Available: https://portal.3gpp.org/desktopmodules/ Specifications/SpecificationDetails.aspx ?specificationId $=3107$

[82] M. M. Ahamed and S. Faruque, "5G Backhaul: Requirements, Challenges, and Emerging Technologies," in Broadband Communications Networks, Sep. 2018, pp. 43-58.

[83] J. Llorca, M. Kalantari, S. Milner, and C. Davis, "A quadratic optimization method for connectivity and coverage control in backbone-based wireless networks," Ad Hoc Networks, vol. 7, pp. 614-621, 052009.

[84] Global System for Mobile Communications (GSMA), "The 5G Guide A Reference For Operators," White Paper, Apr. 2019, available Online. [Online]. Available: https://www.gsma.com/wp-content/uploads/2019/ 04/The-5G-Guide_GSMA_2019_04_29_compressed.pdf

[85] A. K. Majumdar, "Chapter 2 - basics of worldwide broadband wireless access independent of terrestrial limitations," in Optical Wireless Communications for Broadband Global Internet Connectivity, A. K. Majumdar, Ed. Elsevier, 2019, pp. 5 - 38. [Online]. Available: http: //www.sciencedirect.com/science/article/pii/B9780128133651000023

[86] ITU, "Functional requirements for virtual content delivery networks," International Telecommunication Union, Standard, Mar. 2017.

[87] S. Wireless, "Evaluation of LTE-M towards 5G IoT requirements," White Paper, June 2018

[88] A. Saleh, O. Bulakci, S. Redana, and J. Hämäläinen, "On cell range extension in LTE-Advanced type 1 inband relay networks," Wireless Communications and Mobile Computing, vol. 15, 062013.

[89] M. Ding, D. Lopez-Perez, G. Mao, P. Wang, and Z. Lin, "Will the area spectral efficiency monotonically grow as small cells go dense?" in 2015 IEEE Global Communications Conference (GLOBECOM), Dec 2015, pp. 1-7.

[90] M. Ding, P. Wang, D. López-Pérez, G. Mao, and Z. Lin, "Performance impact of LoS and NLoS transmissions in dense cellular networks," IEEE Transactions on Wireless Communications, vol. 15, no. 3, pp. 2365-2380, Mar. 2016.

[91] M. Lauridsen, I. Z. Kovacs, P. Mogensen, M. Sorensen, and S. Holst, "Coverage and capacity analysis of LTE-M and NB-IoT in a rural area," in 2016 IEEE 84th Vehicular Technology Conference (VTC-Fall), Sep. 2016, pp. 1-5.

[92] A. Aloni. Should 5G coverage targets be optimized for mobility? Accessed 2020-01-21. [Online]. Available: https://continualexperience. com/should-5g-coverage-targets-be-optimized-for-mobility

[93] Bundesnetzagentur. Mobile broadband - project 2018 , "5G award - decisions III \& IV of 26 november 2018. Accessed 2020-01-22. [Online]. Available: https://www.bundesnetzagentur.de/EN/Areas/Telecommunications/ \Companies/FrequencyManagement/ElectronicCommunications\ Services/MobileBroadbandProject2018/Project2018.html?nn=324044

[94] M. Shafi, A. F. Molisch, P. J. Smith, T. Haustein, P. Zhu, P. De Silva, F. Tufvesson, A. Benjebbour, and G. Wunder, "5G: A tutorial overview of standards, trials, challenges, deployment, and practice," IEEE Journal on Selected Areas in Communications, vol. 35, no. 6 , pp. 1201-1221, Jun. 2017

[95] J. Arnau, I. Atzeni, and M. Kountouris, "Impact of LOS/NLOS propagation and path loss in ultra-dense cellular networks," in 2016 
IEEE International Conference on Communications (ICC), May 2016, pp. 1-6.

[96] S. Sadr and R. S. Adve, "Handoff rate and coverage analysis in multi-tier heterogeneous networks," IEEE Transactions on Wireless Communications, vol. 14, no. 5, pp. 2626-2638, May 2015.

[97] M. Thurfjell, M. Ericsson, and P. de Bruin, "Network densification impact on system capacity," in 2015 IEEE 81st Vehicular Technology Conference (VTC Spring), May 2015, pp. 1-5.

[98] S. Lee and K. Huang, "Coverage and economy of cellular networks with many base stations," IEEE Communications Letters, vol. 16, no. 7, pp. 1038-1040, Jul. 2012.

[99] M. I. Kamel, W. Hamouda, and A. M. Youssef, "Coverage and capacity analysis with stretched exponential path loss in ultra-dense networks," in GLOBECOM 2017 - 2017 IEEE Global Communications Conference, Dec 2017, pp. 1-6.

[100] A. W. Shaban and M. O. Damen, "Outage probability analysis of heterogeneous relay networks," in 2018 IEEE Wireless Communications and Networking Conference (WCNC), Apr. 2018, pp. 1-6.

[101] O. .Bulakci, A. Saleh, S. Redana, B. Raaf, and J. Hämäläinen, "Enhancing LTE-Advanced relay deployments via relay cell extension," 11 2011

[102] N. S. Networks, "LTE performance for initial deployments," 2008, Berlin.

[103] T. Wirth, V. Venkatkumar, T. Haustein, E. Schulz, and R. Halfmann, "LTE-Advanced relaying for outdoor range extension," in 2009 IEEE 70th Vehicular Technology Conference Fall (VTC), Sep. 2009, pp. 1-4.

[104] N. Okati, B. Razeghi, and M. R. Mosavi, "On relay selection to maximize coverage region for cooperative cellular networks with multiple fixed and unfixed relays," in 2015 6th International Conference on Computing, Communication and Networking Technologies (ICCCNT), Jul. 2015, pp. 1-6.

[105] M. F. Feteiha, M. H. Qutqut, and H. S. Hassanein, "Outage probability analysis of mobile small cells over LTE-A networks," in 2014 International Wireless Communications and Mobile Computing Conference (IWCMC), Aug 2014, pp. 1045-1050.

[106] M. D. Jundhare and A. V. Kulkarni, "An overview and current development of femtocells in 5G technology," in 2016 IEEE International Conference on Advances in Electronics, Communication and Computer Technology (ICAECCT), Dec 2016, pp. 204-209.

[107] M. F. Khan, F. A. Bhatti, A. Habib, S. Jangsher, M. I. Khan, I. Zafar, S. M. Shah, M. A. Jamshed, and J. Iqbal, "Analysis of macro user offloading to femto cells for 5G cellular networks," in 2017 International Symposium on Wireless Systems and Networks (ISWSN), Nov. 2017, pp. 1-6.

[108] N. O, S. Han, and H. Kim, "System capacity and coverage analysis of femtocell networks," in 2010 IEEE Wireless Communication and Networking Conference (WCNC), Apr. 2010, pp. 1-5.

[109] J. G. Andrews, H. Claussen, M. Dohler, S. Rangan, and M. C. Reed, "Femtocells: Past, present, and future," IEEE Journal on Selected Areas in Communications, vol. 30, no. 3, pp. 497-508, Apr. 2012.

[110] S. Landstrom, H. Murai, and A. Simonsson, "Deployment aspects of LTE pico nodes," in 2011 IEEE International Conference on Communications Workshops (ICC), Jun. 2011, pp. 1-5.

[111] B. A. Yasir, G. Su, and N. Bachache, "Range expansion for pico cell in heterogeneous LTE - a cellular networks," in Proceedings of 2012 2nd International Conference on Computer Science and Network Technology (ICCSNT), Dec 2012, pp. 1235-1240.

[112] D. Lopez-Perez, X. Chu, and I. Guvenc, "On the expanded region of picocells in heterogeneous networks," IEEE Journal of Selected Topics in Signal Processing, vol. 6, no. 3, pp. 281-294, Jun. 2012.

[113] K. Vanganuru, S. Ferrante, and G. Sternberg, "System capacity and coverage of a cellular network with D2D mobile relays," in MILCOM 2012 - 2012 IEEE Military Communications Conference, Oct 2012 pp. 1-6.

[114] N. Singh, "Coverage and capacity analysis of relay-based deviceto-device communications underlaid cellular networks," Engineering Science and Technology, an International Journal, 072018.

[115] A. Nauman, M. A. Jamshed, Y. Ahmad, R. Ali, Y. B. Zikria, and S. Won Kim, "An intelligent deterministic D2D communication in narrow-band Internet of Things," in 2019 15th International Wireless Communications Mobile Computing Conference (IWCMC), Jun. 2019, pp. 2111-2115.

[116] L. Melki, S. Najeh, and H. Besbes, "System performance of cellular network underlaying D2D multi-hop communication," in 2015 5th International Conference on Communications and Networking (COMNET), Nov. 2015, pp. 1-5.
[117] H. Qin, Z. Mi, C. Dong, F. Peng, and P. Sheng, "An experimental study on multihop D2D communications based on smartphones," in 2016 IEEE 83rd Vehicular Technology Conference (VTC Spring), May 2016, pp. $1-5$.

[118] W. Qu, G. Li, and Y. Zhao, "On the coverage problem in device-todevice relay networks," IEEE Communications Letters, vol. 23, no. 11, pp. 2139-2143, Nov. 2019.

[119] J. Gui and J. Deng, "Multi-hop relay-aided underlay D2D communications for improving cellular coverage quality," IEEE Access, vol. 6, pp. 14318-14338, 2018.

[120] H. Zhu, "Performance comparison between distributed antenna and microcellular systems," IEEE Journal on Selected Areas in Communications, vol. 29, no. 6, pp. 1151-1163, Jun. 2011.

[121] “Connected Nations 2019 - UK Report,” Ofcom, Tech. Rep., Dec. 2019.

[122] "Further Options for Improving Mobile Coverage - Advice to Government," Ofcom, Tech. Rep., Sep. 2019.

[123] M. S. Department for Digital, Culture. £1 billion deal set to solve poor mobile coverage. Accessed 2020-01-22. [Online]. Available: https://www.gov.uk/government/news/1-billion-deal-set-tosolve-poor-mobile-coverage

[124] "National Inventory of Woodland and Trees: Great Britain," Forestry Commission, Tech. Rep., 2003.

[125] B. Lewis, "Boosting Mobile Connectivity: Written statement HCWS631," Department for Communities and Local Government, Parliamentary Statement, Tech. Rep., Mar. 2016.

[126] S. K. Haider, M. A. Jamshed, A. Jiang, H. Pervaiz, and Q. Ni, "UAVassisted cluster-head selection mechanism for wireless sensor network applications," in 2019 UK/ China Emerging Technologies (UCET) Conference, Aug 2019, pp. 1-2.

[127] J. C.-R. A. Aragón-Zavala and J. Delgado-Penín, High-Altitude Platforms for Wireless Communications, 2nd ed., J. W. . Sons, Ed. WileyBlackwell, 102018.

[128] P. Techavijit, S. Chivapreecha, P. Sukchalerm, and N. Wongphuangfuthaworn, "Suitable altitude for long-operated communication high altitude balloon with experimental flights," in 2016 8th International Conference on Knowledge and Smart Technology (KST), Feb 2016, pp. 169-174.

[129] J. Kelif, "Coverage and performance of stratospheric balloons wireless networks," in 2016 IEEE 27th Annual International Symposium on Personal, Indoor, and Mobile Radio Communications (PIMRC), Sep. 2016, pp. 1-6.

[130] C. Oestges and D. Vanhoenacker-Janvier, "Coverage analysis of a stratospheric communication system," IEE Proceedings - Microwaves, Antennas and Propagation, vol. 148, no. 1, pp. 45-49, Feb 2001.

[131] A. Al-Hourani, S. Kandeepan, and S. Lardner, "Optimal LAP altitude for maximum coverage," IEEE Wireless Communications Letters, vol. 3, no. 6, pp. 569-572, Dec 2014.

[132] S. K. Zaidi, S. F. Hasan, X. Gui, N. Siddique, and S. Ahmad, "Exploiting UAV as NOMA based relay for coverage extension," in 2019 2nd International Conference on Computer Applications Information Security (ICCAIS), May 2019, pp. 1-5.

[133] J. Lyu, Y. Zeng, and R. Zhang, "UAV-aided offloading for cellular hotspot," IEEE Transactions on Wireless Communications, vol. 17, no. 6, pp. 3988-4001, Jun. 2018.

[134] B. G. Evans, "Role of satellites in mobile-wireless systems," in 2004 IEEE 15th International Symposium on Personal, Indoor and Mobile Radio Communications (IEEE Cat. No.04TH8754), vol. 3, Sep. 2004, pp. 2055-2060 Vol.3.

[135] G. Giambene, S. Kota, and P. Pillai, "Satellite-5G integration: A network perspective," IEEE Network, vol. 32, no. 5, pp. 25-31, Sep. 2018.

[136] B. Di, L. Song, Y. Li, and H. V. Poor, "Ultra-dense LEO: Integration of satellite access networks into $5 \mathrm{G}$ and beyond," IEEE Wireless Communications, vol. 26, no. 2, pp. 62-69, Apr. 2019.

[137] S. Cluzel, L. Franck, J. Radzik, S. Cazalens, M. Dervin, C. Baudoin, and D. Dragomirescu, "3GPP NB-IoT coverage extension using LEO satellites," in 2018 IEEE 87th Vehicular Technology Conference (VTC Spring), June 2018, pp. 1-5.

[138] A. Guidotti, A. Vanelli-Coralli, M. Caus, J. Bas, G. Colavolpe, T. Foggi, S. Cioni, A. Modenini, and D. Tarchi, "Satellite-enabled LTE systems in LEO constellations," in 2017 IEEE International Conference on Communications Workshops (ICC Workshops), May 2017, pp. 876881.

[139] G. Hattab, P. Moorut, E. Visotsky, M. Cudak, and A. Ghosh, "Interference analysis of the coexistence of $5 \mathrm{G}$ cellular networks with satellite earth stations in 3.7-4.2GHz," in 2018 IEEE International Conference on Communications Workshops (ICC Workshops), May 2018, pp. 1-6. 
[140] P. V. Klaine, M. A. Imran, O. Onireti, and R. D. Souza, "A survey of machine learning techniques applied to self-organizing cellular networks," IEEE Communications Surveys \& Tutorials, vol. 19, no. 4, pp. 2392-2431, 2017.

[141] C. J. Debono and J. K. Buhagiar, "Cellular network coverage optimization through the application of self-organizing neural networks," in VTC-2005-Fall. 2005 IEEE 62nd Vehicular Technology Conference, 2005., vol. 4, 2005, pp. 2158-2162.

[142] A. Engels, M. Reyer, X. Xu, R. Mathar, J. Zhang, and H. Zhuang, "Autonomous Self-Optimization of Coverage and Capacity in LTE Cellular Networks," IEEE Transactions on Vehicular Technology, vol. 62, no. 5, pp. 1989-2004, 2013.

[143] M. N. ul Islam and A. Mitschele-Thiel, "Reinforcement learning strategies for self-organized coverage and capacity optimization," in 2012 IEEE Wireless Communications and Networking Conference (WCNC), 2012, pp. 2818-2823.

[144] Y. Jo, H. Kim, J. Lim, and D. Hong, "Self-Optimization of Coverage and System Throughput in 5G Heterogeneous Ultra-Dense Networks," IEEE Wireless Communications Letters, vol. 9, no. 3, pp. 285-288, 2020.

[145] H. Claussen, L. T. W. Ho, and L. G. Samuel, "Self-optimization of coverage for femtocell deployments," in 2008 Wireless Telecommunications Symposium, 2008, pp. 278-285.

[146] M. S. Shokry, D. Ebrahimi, C. Assi, S. Sharafeddine, and A. Ghrayeb, "Leveraging UAVs for Coverage in Cell-Free Vehicular Networks: A Deep Reinforcement Learning Approach," IEEE Transactions on Mobile Computing, pp. 1-1, 2020.

[147] U. Challita, H. Ryden, and H. Tullberg, "When machine learning meets wireless cellular networks: Deployment, challenges, and applications," IEEE Communications Magazine, vol. 58, no. 6, pp. 12-18, 2020

[148] J. Moysen and L. Giupponi, "From 4G to 5G: Self-organized Network Management meets Machine Learning," Computer Communications, vol. 129, 072017.

[149] W. Khawaja, O. Ozdemir, Y. Yapici, I. Guvenc, and Y. Kakishima, "Coverage Enhancement for mm-Wave Communications using Passive Reflectors," in 2018 11th Global Symposium on Millimeter Waves (GSMM), May 2018, pp. 1-6.

[150] Z. Peng, L. Li, M. Wang, Z. Zhang, Q. Liu, Y. Liu, and R. Liu, "An effective coverage scheme with passive-reflectors for urban millimeterwave communication," IEEE Antennas and Wireless Propagation Letters, vol. 15, pp. 398-401, 2016.

[151] M. D. Renzo, K. Ntontin, J. Song, F. H. Danufane, X. Qian, F. Lazarakis, J. de Rosny, D. T. Phan-Huy, O. Simeone, R. Zhang, M. Debbah, G. Lerosey, M. Fink, S. Tretyakov, and S. Shamai, "Reconfigurable intelligent surfaces vs. relaying: Differences, similarities, and performance comparison," 2019.

[152] M. Jamshed and F. Jameel. When the IoT meets IRS: Intelligent reflecting surfaces for massive IoT connectivity. Internet of Things Newsletter. Accessed 2020-01-27. [Online]. Available: https://iot.ieee.org/newsletter/january-2020/when-the-iotmeets-irs-intelligent-reflecting-surfaces-for-massive-iot-connectivity

[153] Q. Wu and R. Zhang, "Intelligent reflecting surface enhanced wireless network: Joint active and passive beamforming design," in 2018 IEEE Global Communications Conference (GLOBECOM), Dec 2018, pp. 1-

[154] Q. Nadeem and A. Kammoun and A. Chaaban and M. Debbah and M. Alouini, "Asymptotic max-min SINR analysis of reconfigurable intelligent surface assisted MISO systems," 2019.

[155] D. Lopez-Perez, I. Guvenc, G. de la Roche, M. Kountouris, T. Q. S. Quek, and J. Zhang, "Enhanced intercell interference coordination challenges in heterogeneous networks," IEEE Wireless Communications, vol. 18, no. 3, pp. 22-30, Jun. 2011.

[156] Q. Zhang, W. Saad, and M. Bennis, "Reflections in the sky: Millimeter wave communication with uav-carried intelligent reflectors," in IEEE Global Communications Conference (GLOBECOM), 12 2019, pp. 1-6.

[157] D. Ma, M. Ding, and M. Hassan, "Enhancing Cellular Communications for UAVs via Intelligent Reflective Surface," in IEEE Wireless Communications and Networking Conference (WCNC), 05 2020, pp. $1-6$.

[158] L. Ge, P. Dong, H. Zhang, J. Wang, and X. You, "Joint Beamforming and Trajectory Optimization for Intelligent Reflecting SurfacesAssisted UAV Communications," IEEE Access, vol. 8, pp. 78702 $78712,2020$.

[159] K. Tekbiyik, G. K. Kurt, A. Ekti, and A. Görçin, "Reconfigurable Intelligent Surface Empowered Terahertz Communication for LEO Satellite Networks," submitted to IEEE Journal on Selected Areas in Communications, 072020.
[160] G. Interdonato, E. Björnson, H. Ngo, P. Frenger, and E. Larsson, "Ubiquitous cell-free massive MIMO communications," EURASIP Journal on Wireless Communications and Networking, 082019.

[161] E. Björnson and L. Sanguinetti, "Making cell-free massive MIMO competitive with MMSE processing and centralized implementation," IEEE Transactions on Wireless Communications, vol. 19, no. 1, pp. 77-90, 2020

[162] V. Jungnickel, K. Manolakis, W. Zirwas, B. Panzner, V. Braun, M. Lossow, M. Sternad, R. Apelfröjd, and T. Svensson, "The role of small cells, coordinated multipoint, and massive MIMO in 5G," IEEE Communications Magazine, vol. 52, no. 5, pp. 44-51, May 2014.

[163] D. Castanheira, P. Lopes, A. Silva, and A. Gameiro, "Hybrid Beamforming Designs for Massive MIMO Millimeter-Wave Heterogeneous Systems," IEEE Access, vol. 5, pp. 21 806-21 817, 2017.

[164] S. L. Bernstein, M. L. Burrows, J. E. Evans, A. S. Griffiths, D. A. McNeill, C. W. Niessen, I. Richer, D. P. White, and D. K. Willim, "Long-range communications at extremely low frequencies," Proceedings of the IEEE, vol. 62, no. 3, pp. 292-312, Mar. 1974.

[165] H. Mott and A. Biggs, "Very-low-frequency propagation below the bottom of the sea," IEEE Transactions on Antennas and Propagation, vol. 11, no. 3, pp. 323-329, May 1963.

[166] S. University. Introduction to VLF," Stanford VLF group. Accessed 2020-01-09. [Online]. Available: https://vlf.stanford.edu/ research/introduction-vlf

[167] M. Cohen, M. Golkowski, and U. Inan, "Orientation of the HAARP ELF ionospheric dipole and the auroral electrojet," Geophys. Res. Lett., vol. 35, p. doi:10.1029/2007GL032424, 012008.

[168] R. Moore, U. Inan, T. Bell, and E. Kennedy, "ELF waves generated by modulated HF heating of the auroral electrojet and observed at a ground distance of approximately $4400 \mathrm{~km}$, , Journal of Geophysical Research (Space Physics), vol. 112, p. 8, 052007.

[169] J. Wang, G. Ding, and H. Wang, "HF communications: Past, present, and future," China Communications, vol. 15, no. 9, pp. 1-9, Sep. 2018

[170] A. A. Barrios, "High-frequency skywave communication system performance in a nuclear environment," IEEE Transactions on Military Electronics, vol. 9, no. 2, pp. 125-130, Apr. 1965.

[171] F. Orga, M. Hervas, and R. M. Alsina-Pages, "Flexible low-cost SDR platform for HF communications: Near vertical incidence skywave preliminary results." IEEE Antennas and Propagation Magazine, vol. 58, no. 6, pp. 49-56, Dec. 2016.

[172] T. Thayaparan, Y. Ibrahim, J. Polak, and R. Riddolls, "High-frequency over-the-horizon radar in Canada," IEEE Geoscience and Remote Sensing Letters, vol. 15, no. 11, pp. 1700-1704, Nov. 2018.

[173] R. L. Frank, "Current developments in Loran-C," Proceedings of the IEEE, vol. 71, no. 10, pp. 1127-1139, Oct. 1983.

[174] G. Ancāns, V. Bobrovs, A. Ancans, and D. Kalibatiene, "Spectrum considerations for 5G mobile communication systems," Procedia Computer Science, vol. 104, pp. 509-516, 122017.

[175] SmartMakers. (2019) LoRaWAN range, part 1: The most important factors for a good LoRaWAN signal range. Accessed 2020-01-27. [Online]. Available: https://smartmakers.io/en/lorawan-range-part-1the-most-important-factors-for-a-good-lorawan-signal-rang

[176] J. Haxhibeqiri, E. D. Poorter, I. Moerman, and J. Hoebeke, "A survey of LoRaWAN for IoT: From technology to application," Sensors, vol. 18 , p. 3995,112018

[177] A. Augustin, J. Yi, T. Clausen, and W. William, "A study of LoRa: Long range \& low power networks for the Internet of Things," Sensors, vol. 16, p. 1466, 102016.

[178] J. Petajajarvi, K. Mikhaylov, A. Roivainen, T. Hanninen, and M. Pettissalo, "On the coverage of LPWANs: range evaluation and channel attenuation model for LoRa technology," in 2015 14th International Conference on ITS Telecommunications (ITST), Dec 2015, pp. 55-59.

[179] B. Vejlgaard, M. Lauridsen, H. Nguyen, I. Z. Kovacs, P. Mogensen, and M. Sorensen, "Coverage and capacity analysis of Sigfox, LoRa, GPRS, and NB-IoT," in 2017 IEEE 85th Vehicular Technology Conference (VTC Spring), Jun. 2017, pp. 1-5.

[180] M. Lauridsen, H. Nguyen, B. Vejlgaard, I. Z. Kovacs, P. Mogensen, and M. Sorensen, "Coverage comparison of GPRS, NB-IoT, LoRa, and Sigfox in a $7800 \mathrm{~km}^{2}$ area," in 2017 IEEE 85th Vehicular Technology Conference (VTC Spring), Jun. 2017, pp. 1-5.

[181] J. Finnegan and S. Brown, "A comparative survey of LPWA networking," 2018. [Online]. Available: https://arxiv.org/pdf/1802. 04222.pdf

[182] S. Rangan, T. S. Rappaport, and E. Erkip, "Millimeter-wave cellular wireless networks: Potentials and challenges," Proceedings of the IEEE, vol. 102 , no. 3, pp. 366-385, Mar. 2014. 
[183] T. Bai and R. W. Heath, "Coverage and rate analysis for millimeterwave cellular networks," IEEE Transactions on Wireless Communications, vol. 14, no. 2, pp. 1100-1114, Feb 2015.

[184] S. Wu, R. Atat, N. Mastronarde, and L. Liu, "Improving the coverage and spectral efficiency of millimeter-wave cellular networks using device-to-device relays," IEEE Transactions on Communications, vol. 66, no. 5, pp. 2251-2265, May 2018.

[185] J. Lee, J. Choi, and S. Kim, "Cell Coverage Analysis of $28 \mathrm{GHz}$ Millimeter Wave in Urban Microcell Environment Using 3-D Ray Tracing," IEEE Transactions on Antennas and Propagation, vol. 66, no. 3, pp. 1479-1487, Mar. 2018.

[186] K. Cho and C. G. Kang, "Coverage analysis of cellular network with bidirectional beamforming," in 2018 International Conference on Information and Communication Technology Convergence (ICTC), Oct 2018, pp. 754-756.

[187] X. Yu, J. Zhang, M. Haenggi, and K. B. Letaief, "Coverage analysis for millimeter wave networks: The impact of directional antenna arrays," IEEE Journal on Selected Areas in Communications, vol. 35, no. 7, pp. 1498-1512, Jul. 2017.

[188] Y. Zhou, V. W. S. Wong, and R. Schober, "Coverage and rate analysis of millimeter wave NOMA networks with beam misalignment," IEEE Transactions on Wireless Communications, vol. 17, no. 12, pp. 82118227, Dec. 2018.

[189] M. Leba, S. Riurean, and A. Lonica, "LiFi - The path to a new way of communication," in 2017 12th Iberian Conference on Information Systems and Technologies (CISTI), 2017, pp. 1-6.

[190] D. Tsonev, S. Videv, and H. Haas, "Light fidelity (Li-Fi): Towards all-optical networking," vol. 9007, 12 2013, p. 900702.

[191] H. Haas, L. Yin, C. Chen, S. Videv, D. Parol, E. Poves, H. Alshaer, and M. S. Islim, "Introduction to indoor networking concepts and challenges in LiFi," IEEE/OSA Journal of Optical Communications and Networking, vol. 12, no. 2, pp. A190-A203, 2020.

[192] N. S. et al. Light Communications for Wireless Local Area Networking. New Future Networks Tech Focus (Online). Accessed 2020-09-03. [Online]. Available: https://futurenetworks.ieee.org/tech-focus/may2018/light-communications-for-wireless-local-area-networking

[193] “A Framework for Spectrum Sharing," Ofcom, Tech. Rep., Jul. 2015.

[194] L. Wan, Z. Guo, Y. Wu, W. Bi, J. Yuan, M. Elkashlan, and L. Hanzo, " $4 \mathrm{G} / 5 \mathrm{G}$ spectrum sharing: Efficient $5 \mathrm{G}$ deployment to serve enhanced mobile broadband and Internet of Things applications," IEEE Vehicular Technology Magazine, vol. 13, no. 4, pp. 28-39, Dec. 2018.

[195] E. Hossain, M. Rasti, H. Tabassum, and A. Abdelnasser, "Evolution toward 5G multi-tier cellular wireless networks: An interference management perspective," IEEE Wireless Communications, vol. 21, no. 3, pp. 118-127, Jun. 2014.

[196] E. Pateromichelakis, H. Celik, R. Fantini, O. Bulakci, L. M. Campoy, D. M. Gutierrez-Estevez, A. M. Ibrahim, F. J. Lorca, M. Shariat, M. Tesanovic, and Y. Yang, "Interference management enablers for 5G radio access networks," in 2016 IEEE Conference on Standards for Communications and Networking (CSCN), Oct 2016, pp. 1-7.

[197] M. Yassin, M. Aboulhassan, S. Lahoud, M. Ibrahim, D. Mezher, B. Cousin, and E. Sourour, "Survey of ICIC Techniques in LTE Networks under Various Mobile Environment Parameters," Wireless Networks, vol. 23, pp. 403-418, 022017.

[198] S. Chang, H. Park, S. Kim, and J. P. Choi, "Study on coverage of full frequency reuse in FFR systems based on outage probability," IEEE Transactions on Communications, vol. 66, no. 11, pp. 5828-5843, Nov. 2018.

[199] Z. Xie and B. Walke, "Frequency reuse techniques for attaining both coverage and high spectral efficiency in OFDMA cellular systems," in 2010 IEEE Wireless Communication and Networking Conference (WCNC), Apr. 2010, pp. 1-6.

[200] N. Katiran, N. Fisal, S. Kamilah, S. K. Yusof, S. Marwangi, M. Maharum, A. Syamimi, A. Ghafar, and F. Saparudin, "Inter-cell Interference Mitigation and Coordination in CoMP Systems," vol. 253, 11 2011.

[201] W. Liu, C. Hu, D. Wei, M. Peng, and W. Wang, "An overload indicator $\&$ high interference indicator hybrid scheme for inter-cell interference coordination in LTE system," in 2010 3rd IEEE International Conference on Broadband Network and Multimedia Technology (IC-BNMT), Oct. 2010, pp. 514-518.

[202] Z. Wang, W. Xiong, C. Dong, J. Wang, and S. Li, "A novel downlink power control scheme in LTE heterogeneous network," in 2011 International Conference on Computational Problem-Solving (ICCP), Oct. 2011, pp. 241-245.
[203] T. Hassan and F. Gao, "An active power control technique for downlink interference management in a two-tier macro-femto network," Sensors, vol. 19, p. 2015, 042019.

[204] G. Jaheon, B. Sueng Jae , C. Bum-Gon , and M. Young Chung, "Dynamic power control mechanism for interference coordination of device-to-device communication in cellular networks," in 2011 Third International Conference on Ubiquitous and Future Networks (ICUFN), Jun. 2011, pp. 71-75.

[205] J. F. Shi, M. Chen, Z. H. Yang, H. Xu, and Y. Wang, "Power control and performance analysis for full-duplex relay-assisted D2D communication underlaying fifth generation cellular networks," IET Communications, vol. 11, no. 18, pp. 2729-2734, 2017.

[206] J. Oh and Y. Han, "Cell selection for range expansion with almost blank subframe in heterogeneous networks," in 2012 IEEE 23rd International Symposium on Personal, Indoor and Mobile Radio Communications (PIMRC), Sep. 2012, pp. 653-657.

[207] H. Jang and W. Wend, "Interference management using frequency reuse and CoMP for LTE-Advanced networks," in 2015 Seventh International Conference on Ubiquitous and Future Networks (ICUFN), Jul. 2015, pp. $740-745$.

[208] D. Hai-Tao, Z. Wen-An, L. Xian-Qi, and S. Jing-Yuan, "An optimized resource allocation and CoMP based interference coordination scheme for LTE-A Het-Net," in 2014 Ninth International Conference on Broadband and Wireless Computing, Communication and Applications (BWCCA), Nov. 2014, pp. 207-211.

[209] A. Papazafeiropoulos, P. Kourtessis, M. D. Renzo, S. Chatzinotas, and J. M. Senior, "Performance analysis of cell-free massive MIMO systems: A stochastic geometry approach," IEEE Transactions on Vehicular Technology, vol. 69, no. 4, pp. 3523-3537, 2020.

[210] O. Dizdar, Y. Mao, W. Han, and B. Clerckx, "Rate-Splitting Multiple Access: A New Frontier for the PHY Layer of 6G," 06 2020. [Online]. Available: https://arxiv.org/abs/2006.01437

[211] B. Clerckx, H. Joudeh, C. Hao, M. Dai, and B. Rassouli, "Rate splitting for MIMO wireless networks: a promising PHY-layer strategy for LTE evolution," IEEE Communications Magazine, vol. 54, no. 5, pp. 98$105,2016$.

[212] A. A. Ahmad, J. Kakar, R. Reifert, and A. Sezgin, "UAV-Assisted CRAN with Rate Splitting Under Base Station Breakdown Scenarios," in 2019 IEEE International Conference on Communications Workshops (ICC Workshops), 2019, pp. 1-6.

[213] A. Alameer Ahmad, H. Dahrouj, A. Chaaban, A. Sezgin, and M. Alouini, "Interference mitigation via rate-splitting in cloud radio access networks," in IEEE 19th International Workshop on Signal Processing Advances in Wireless Communications (SPAWC), 2018, pp. $1-5$.

[214] E. Baştuğ, M. Bennis, M. Kountouris, and M. Debbah, "Edge caching for coverage and capacity-aided heterogeneous networks," in IEEE International Symposium on Information Theory (ISIT), 2016, pp. 285289.

[215] M. Falk, R. A. Justavino C., G. Bauch, and E. Seidel, "Partial Interference Cancellation in Heterogeneous LTE-Advanced Networks," in 11th International ITG Conference on Systems, Communications and Coding (SCC), 2017, pp. 1-6.

[216] B. Clerckx, "Rate-Splitting and Robust Interference Management: Theory and Applications," in IEEE PIMRC 2018; International Symposium on Personal, Indoor and Mobile Radio Communications, 2018 , Tutorials.

[217] N. Naderializadeh, O. Orhan, H. Nikopour, and S. Talwar, "Ultra-dense networks in 5G: Interference management via non-orthogonal multiple access and treating interference as noise," in 2017 IEEE 86th Vehicular Technology Conference (VTC-Fall), Sep. 2017, pp. 1-6.

[218] C. Liu, D. Liang, P. Chen, and J. Yang, "Coverage analysis for dense heterogeneous networks with cooperative NOMA," in 2017 IEEE 85th Vehicular Technology Conference (VTC Spring), Jun. 2017, pp. 1-6.

[219] C. Liu and D. Liang, "Heterogeneous networks with power-domain NOMA: Coverage, throughput, and power allocation analysis," IEEE Transactions on Wireless Communications, vol. 17, no. 5, pp. 3524 3539, May 2018. 


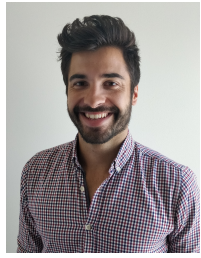

Rúben Borralho received the Masters degree in electrical and computer engineering from the Instituto Superior Técnico, University of Lisbon, Lisbon, Portugal, in 2017. He worked in the telecommunications industry for 18 months, as a research lead in the Portuguese telecommunications company Celfinet's R\&D department, being involved in P2020 collaborative research projects, namely: Non GPS Assisted Positioning for Mobile Networks in Indoor and Outdoor Environments and Mobile Networks Energy Efficient Automatic Orchestration for Optimized Quality of Experience. He is currently pursuing the $\mathrm{Ph} . \mathrm{D}$. degree with the Institute of Communication Systems (ICS) at the University of Surrey, U.K. He takes part in the MAC, RRM and RAN management work area. His main areas of research interest include radio access networks planning and optimization, and geopositioning. In parallel, he also praises the programming component in his work, which is part of his background.

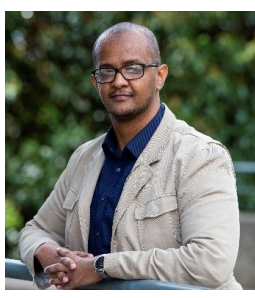

Abdelrahim Mohamed (S'15-M'16) received the B.Sc. degree (First Class) in electrical and electronics engineering from the University of Khartoum, Sudan, in 2011, the M.Sc. degree (distinction) in mobile and satellite communications, and the Ph.D. degree in electronics engineering from the University of Surrey, U.K., in 2013 and 2016 respectively. He is currently a Postdoctoral Research Fellow with the 5G Innovation Centre (5GIC), U.K. He is currently involved in the RRM, MAC and RAN Management work area, and the New Physical Layer work area in the 5GIC. He worked on multiple projects including: Energy Proportional eNB for LTE-Advanced and Beyond, U.K. DCMS Planning Tool for 5G Network in mm-wave Band project, EPSRC Stepping Towards the Industrial Sixth Sense, and $5 \mathrm{G}$ Non-Terrestrial Networks in parallel to working on the development of the 5G System Level Simulator. His main areas of research interest include radio access network design, system-level simulations and analysis, mobility management, energy efficiency and cognitive radio. He secured first place and top ranked in the Electrical and Electronic Engineering Department, University of Surrey, U.K. during his M.Sc. studies. He was a recipient of the Sentinels of Science 2016 Award.

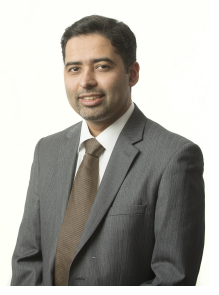

Atta UI Quddus received the M.Sc. degree in satellite communications and the Ph.D. degree in mobile cellular communications from the University of Surrey, U.K., in 2000 and 2005, respectively. During his research career, he has led several national and international research projects that also contributed toward 3GPP standardization. He is currently a Lecturer in wireless communications with the Institute of Communications, Department of Electronic Engineering, University of Surrey. His research interests include radio access network (RAN) deployment strategies, machine type communication, and device to device communication. In 2004, he received the Centre for Communications Systems Research Excellence Prize sponsored by Vodafone for his research on adaptive filtering algorithms. He is a Fellow of Higher Education Academy U.K.

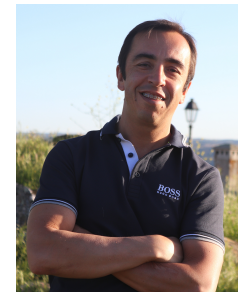

Pedro Vieira was born in Lisbon, Portugal, in 1974. He received the Eng., the M.Sc. and the Ph.D. degree in Electrical and Computer Engineering from Instituto Superior Técnico (IST), Technical University of Lisbon, Portugal, in 1997, 2003 and 2008, respectively. Since 1997, he has been with the Electronics, Telecommunications and Computer Engineering Department, in the Lisbon Polytechnic Institute (ISEL). $\mathrm{He}$ is currently an Adjunct Professor in ISEL and a Researcher at Instituto de Telecomunicações, where he is researching aspects of wireless communications, including radio propagation, radio network planning and optimization and SON systems. He is also a senior engineer registered in the Portuguese Engineering Order (OE), and a OE telecommunications specialist, since 2008. Currently, he is leading the research activities in CELFINET, a Portuguese technological consultancy company, where he is engaging in applied research to create new methodologies, tools and algorithms for the mobile communications business.

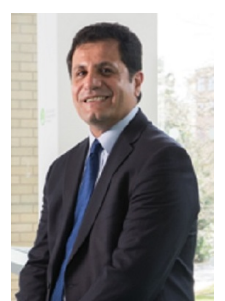

Rahim Tafazolli (SM'09) is a professor and the Director of the Institute for Communication Systems (ICS) and 5G Innovation Centre (5GIC), the University of Surrey in the U.K.. He has published more than 500 research papers in refereed journals, international conferences and as invited speaker. $\mathrm{He}$ is the editor of two books on "Technologies for Wireless Future" published by Wiley's Vol.1 in 2004 and Vol.2 2006. He was appointed as Fellow of WWRF (Wireless World Research Forum) in April 2011, in recognition of his personal contribution to the wireless world. As well as heading one of Europe's leading research groups. 